\title{
Radical Hydroxyalkylation of C-H Bond Adjacent to Nitrogen of Tertiary Amides, Ureas, and Amines
}

\author{
Takehiko Yoshimitsu, * Yoshimasa Arano, and Hiroto Nagaoka* \\ Meiji Pharmaceutical University, 2-522-1 Noshio, Kiyose, Tokyo 204-8588, Japan \\ takey@my-pharm.ac.jp
}

\section{Supporting Information}

Table of Contents

General Procedures:

S2

Compounds 3a, 4a, and 5a: S2-3

Compounds 3b, 4b, and 4b: S3-4

Compounds $\mathbf{3 c}, \mathbf{4 c}$, and $\mathbf{4 c :} \quad$ S4-5

Compounds 3d, 4d, and 5d: S5-6

Compounds 3e and 4e: $\quad$ S7

Compounds 16 and 17: $\quad$ S7-8

Compounds 18 and 19: $\quad$ S9-10

Compounds 20 and 21: $\quad$ S10-11

Compounds 22:

S11

Compounds 23:

S12

Compounds 24 and 25:

S12-13

Compounds 26 and 27:

S13-14

Compounds 28:

S14-15
${ }^{1} \mathrm{H}$ NMR $/{ }^{13} \mathrm{C}$ NMR Spectra: $\quad$ S16-S96

CIF files:

(1) Major threo-3a

(2) 4-Bromobenzoate derived from threo-3c

(3) Minor erythro-16

(4) Minor erythro-18

(5) Major threo-26 
General procedures. All reagents were used as received from commercial suppliers unless otherwise noted. 4-Methoxybenzaldehyde, benzaldehyde, triethylamine, 3-methyl-2-oxazolidinone, and $N, N$-dimethylacetamide were purified by distillation prior to use. ${ }^{1} \mathrm{H}$ NMR spectra (400 or $300 \mathrm{MHz}$ ) and ${ }^{13} \mathrm{C}$ NMR spectra $(100$ or $75 \mathrm{MHz})$ were measured in $\mathrm{CDCl}_{3}$. Chemical shifts are reported in ppm relative to the internal solvent signal; $\mathrm{CHCl}_{3}(7.26 \mathrm{ppm})$ for ${ }^{1} \mathrm{H}$ NMR and $\mathrm{CDCl}_{3}(77.0 \mathrm{ppm})$ for ${ }^{13} \mathrm{C}$ NMR. FT-IR spectra were recorded for samples loaded as neat films on $\mathrm{NaCl}$ plates. Mass spectra were obtained according to the specified technique. Analytical thin layer chromatography (TLC) was performed using Kieselgel $60 \mathrm{~F}_{254}$, and compounds were visualized with UV light, anisaldehyde stain, phosphomolybdic acid in $\mathrm{EtOH}$, iodine, or $\mathrm{KMnO}_{4}$ stain.

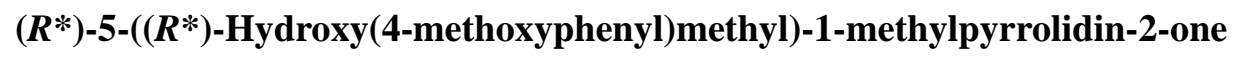
(3a), $\left(R^{*}\right)$-5-((S*)-Hydroxy(4-methoxyphenyl)methyl)-1-methylpyrrolidin-2-one

(4a), and 1-(2-Hydroxy-2-(4-methoxyphenyl)ethyl)pyrrolidin-2-one (5a): To a solution of 4-methoxybenzaldehyde (2a) (408 mg, $3.0 \mathrm{mmol})$ in 1-methyl-2-pyrrolidinone (1) (10 mL, $105 \mathrm{mmol})$ was added $\mathrm{Et}_{3} \mathrm{~B}(2.6 \mathrm{~mL}, 18 \mathrm{mmol})$ at room temperature under argon atmosphere. After removal of the argon balloon, the mixture was stirred at the same temperature with continuous bubbling of air through a syringe needle with a balloon [flow rate; ca. $30 \mathrm{~mL} \cdot \mathrm{h}^{-1} \cdot \mathrm{mmol}$ aldehyde $\mathrm{e}^{-1}$ ] for $14 \mathrm{~h}$. Sat. $\mathrm{NH}_{4} \mathrm{Cl}$ solution was added and the mixture was extracted with $\mathrm{CH}_{2} \mathrm{Cl}_{2}$. The organic extract was dried over $\mathrm{MgSO}_{4}$ and concentrated in vacuo. The residue was purified by silica gel column chromatography (EtOAc/Hex 1:4) to give ethyl adduct 6a $(29 \mathrm{mg}, 6 \%)$ as a colorless oil. Further elution with $\mathrm{MeOH} /$ EtOAc (1:40) gave a mixture of alcohols 3a, 4a, and 5a (527 mg, 75\%) as a colorless solid. The yields and the ratio of the products were determined by ${ }^{1} \mathrm{H}$ NMR analysis of the above mixture to be $\mathbf{3 a} / \mathbf{4 a}(480 \mathrm{mg}, 68 \%$, dr $68: 32)$ and $\mathbf{5 a}(47 \mathrm{mg}, 7 \%)$. Recrystallization of the mixture from EtOAc/ $\mathrm{CH}_{2} \mathrm{Cl}_{2}$ gave 3a and $\mathbf{4 a}$, both as colorless prisms. A pure sample of alcohol 5a was obtained as follows: acetylation of the residue obtained by the concentration of the mother liquor that originated from recrystallization $\left(\mathrm{Ac}_{2} \mathrm{O}, \mathrm{Et}_{3} \mathrm{~N}\right.$, DMAP, $\mathrm{CH}_{2} \mathrm{Cl}_{2}$, r.t.), chromatographic separation ( $\mathrm{MeOH} / \mathrm{EtOAc}$ 1:50), and hydrolysis of the acetates $\left(\mathrm{K}_{2} \mathrm{CO}_{3}\right.$, aq. $\mathrm{MeOH}$, r.t.). Alcohol 6a was identical to commercially available 1-(4-methoxypheyl)propan-1-ol. The relative configuration of major product 
3a was determined to be threo by X-ray crystallographic analysis; details are provided in the CIF file accompanying this Supporting Information. threo-Alcohol 3a: $\mathrm{Mp} 157-158{ }^{\circ} \mathrm{C}\left(\mathrm{EtOAc} / \mathrm{CH}_{2} \mathrm{Cl}_{2}\right)$; IR (neat) $v 3371,1664 \mathrm{~cm}^{-1} ;{ }^{1} \mathrm{H}$ NMR $\left(300 \mathrm{MHz}, \mathrm{CDCl}_{3}\right) \delta$ 7.23-7.17 (m, 2H), 6.88-6.83 (m, 2H), $4.68(\mathrm{~d}$, $1 \mathrm{H}, J=5.9 \mathrm{~Hz}), 3.78(\mathrm{~s}, 3 \mathrm{H}), 3.75(\mathrm{~m}, 1 \mathrm{H}), 2.94(\mathrm{~s}, 3 \mathrm{H}), 2.77(\mathrm{br}, 1 \mathrm{H}), 2.06-1.64(\mathrm{~m}, 4 \mathrm{H}) ;{ }^{13} \mathrm{C} \mathrm{NMR}$ $\left(75 \mathrm{MHz}, \mathrm{CDCl}_{3}\right) \delta 176.0,159.4,132.7,127.4,113.9,75.1,65.3,55.2,30.2,29.6,20.9 ; \mathrm{MS} \mathrm{m} / \mathrm{z}: 235$ $\left(\mathrm{M}^{+}\right), 98$ (100\%); HRMS (EI) calcd for $\mathrm{C}_{13} \mathrm{H}_{17} \mathrm{O}_{3} \mathrm{~N}\left(\mathrm{M}^{+}\right)$: 235.1208, found: 235.1203. erythro-Alcohol 4a: $\mathrm{Mp} \mathrm{143-144}{ }^{\circ} \mathrm{C}\left(\mathrm{EtOAc} \mathrm{CH}_{2} \mathrm{Cl}_{2}\right)$; IR (neat) $v 3371,1666 \mathrm{~cm}^{-1} ;{ }^{1} \mathrm{H} \mathrm{NMR}\left(300 \mathrm{MHz}, \mathrm{CDCl}_{3}\right) \delta$ 7.31-7.25 (m, 2H), 6.93-6.87 (m, 2H), 4.97 (d, 1H, J=3.0 Hz), 3.81 (s, 3H), 3.70 (dt, 1H, J=8.6, 3.0 $\mathrm{Hz}), 2.90(\mathrm{~s}, 3 \mathrm{H}), 2.37-2.12(\mathrm{~m}, 2 \mathrm{H}), 2.00(\mathrm{~m}, 1 \mathrm{H}), 1.76(\mathrm{~m}, 1 \mathrm{H}) ;{ }^{13} \mathrm{C} \mathrm{NMR}\left(75 \mathrm{MHz}, \mathrm{CDCl}_{3}\right) \delta 176.4$, 159.2, 132.2, 127.2, 113.9, 71.9, 66.0, 55.3, 30.1, 28.8, 18.3; MS m/z: $235\left(\mathrm{M}^{+}\right), 98(100 \%)$; HRMS (EI) calcd for $\mathrm{C}_{13} \mathrm{H}_{17} \mathrm{O}_{3} \mathrm{~N}\left(\mathrm{M}^{+}\right)$: 235.1208, found: 235.1212 .

1-(2-Hydroxy-2-(4-methoxyphenyl)ethyl)pyrrolidin-2-one (5a): IR (neat) $v 3383,1664 \mathrm{~cm}^{-1} ;{ }^{1} \mathrm{H}$ NMR (400 MHz, $\left.\mathrm{CDCl}_{3}\right) \delta$ 7.32-7.29 (m, 2H), 6.91-6.87 (m, 2H), $4.92(\mathrm{dd}, 1 \mathrm{H}, J=7.0,4.0 \mathrm{~Hz}), 3.81$ (s, $3 \mathrm{H}), 3.55(\mathrm{dd}, 1 \mathrm{H}, J=14.0,7.0 \mathrm{~Hz}), 3.49$ (dd, 1H, J=14.0, $4.0 \mathrm{~Hz}), 3.35(\mathrm{~m}, 1 \mathrm{H}), 3.23(\mathrm{~m}, 1 \mathrm{H}), 2.41$ (t, $2 \mathrm{H}, J=8.2 \mathrm{~Hz}), 2.03-1.94(\mathrm{~m}, 2 \mathrm{H}) ;{ }^{13} \mathrm{C} \mathrm{NMR}\left(100 \mathrm{MHz}, \mathrm{CDCl}_{3}\right) \delta 177.0,159.1,134.0,126.9,113.8$, 72.9, 55.3, 52.0, 49.6, 30.9, 18.5; MS m/z: $235\left(\mathrm{M}^{+}\right), 99$ (100\%); HRMS (EI) calcd for $\mathrm{C}_{13} \mathrm{H}_{17} \mathrm{O}_{3} \mathrm{~N}$ $\left(\mathrm{M}^{+}\right)$: 235.1208, found: 235.1207 .

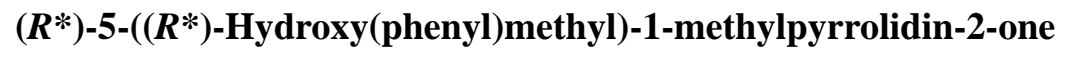

(3b), $\left(R^{*}\right)-5-\left(\left(S^{*}\right)-\right.$ Hydroxy(phenyl)methyl)-1-methylpyrrolidin-2-one

(4b), and 1-(2-Hydroxy-2-phenylethyl)pyrrolidin-2-one (5b): The title compounds were obtained according to the above-mentioned procedure from benzaldehyde (2b) $(318 \mathrm{mg}, \quad 3.0 \mathrm{mmol})$, 1-methyl-2-pyrrolidinone (1) $(10 \mathrm{~mL}, 105 \mathrm{mmol})$ and $\mathrm{Et}_{3} \mathrm{~B}(2.6 \mathrm{~mL}, 18 \mathrm{mmol})$. Reaction time: $16 \mathrm{~h}$. Purification by silica gel column chromatography (EtOAc/Hex 1:5) gave benzyl alcohol (7b) (13 mg, $4 \%)$ as a colorless oil. Further elution with $\mathrm{MeOH} /$ EtOAc (1:40) gave a mixture of alcohols $\mathbf{3 b}, \mathbf{4 b}$, and $\mathbf{5 b}(435 \mathrm{mg}, 71 \%)$ as a colorless solid. The yields and the ratio of the products were determined by ${ }^{1} \mathrm{H}$ NMR analysis to be $\mathbf{3 b} / \mathbf{4 b}$ (379 mg, 62\%, dr 67:33) and $\mathbf{5 b}$ (56 mg, 9\%). Recrystallization of the mixture from EtOAc/ $\mathrm{CH}_{2} \mathrm{Cl}_{2}$ gave $\mathbf{3 b}$ as colorless prisms and $\mathbf{4 b}$ as colorless pillars. The relative 
configuration of major product $\mathbf{3 b}$ was determined by its transformation into a known compound; $\mathbf{3 b}$ was reduced with LAH in 1,4-dioxane to provide $\left(R^{*}\right)$-phenyl(( $\left.R^{*}\right)$-1-methylpyrrolidin-2-yl)methanol (93\%) whose spectroscopic and analytical data were identical with those reported (See reference 1 in S15). threo-Alcohol 3b: $\mathrm{Mp} 138-139{ }^{\circ} \mathrm{C}\left(\mathrm{EtOAc} / \mathrm{CH}_{2} \mathrm{Cl}_{2}\right)$; IR (neat) $v 3339,1666 \mathrm{~cm}^{-1} ;{ }^{1} \mathrm{H}$ NMR $(300$ $\left.\mathrm{MHz}, \mathrm{CDCl}_{3}\right) \delta$ 7.36-7.25 (m, 5H), $4.72(\mathrm{~d}, 1 \mathrm{H}, J=5.7 \mathrm{~Hz}), 3.76(\mathrm{~m}, 1 \mathrm{H}), 3.08(\mathrm{br}, 1 \mathrm{H}), 2.91(\mathrm{~s}, 3 \mathrm{H})$, 2.24-1.66 (m, 4H); ${ }^{13} \mathrm{C}$ NMR (75 MHz, $\left.\mathrm{CDCl}_{3}\right) \delta 176.0,140.7,128.5,128.1,126.3,75.4,65.3,30.2$, 29.6, 20.9; MS m/z: $205\left(\mathrm{M}^{+}\right), 98(100 \%)$; HRMS (EI) calcd for $\mathrm{C}_{12} \mathrm{H}_{15} \mathrm{O}_{2} \mathrm{~N}\left(\mathrm{M}^{+}\right)$: 205.1103, found: 205.1100. erythro-Alcohol 4b: $\mathrm{Mp} 117-118{ }^{\circ} \mathrm{C}\left(\mathrm{EtOAc} / \mathrm{CH}_{2} \mathrm{Cl}_{2}\right)$; IR (neat) $v 3352,1668 \mathrm{~cm}^{-1} ;{ }^{1} \mathrm{H}$ NMR (400 MHz, $\left.\mathrm{CDCl}_{3}\right) \delta$ 7.40-7.26 (m, 5H), $5.06(\mathrm{t}, 1 \mathrm{H}, J=3.0 \mathrm{~Hz}), 3.72(\mathrm{dt}, 1 \mathrm{H}, J=8.8,3.0 \mathrm{~Hz})$, 3.45 (br, 1H), $2.93(\mathrm{~s}, 3 \mathrm{H}), 2.37$ (m, 1H), $2.20(\mathrm{ddd}, 1 \mathrm{H}, J=17.0,10.4,4.2 \mathrm{~Hz}), 2.03$ (m, 1H), 1.73 (m, $1 \mathrm{H}) ;{ }^{13} \mathrm{C} \mathrm{NMR}\left(100 \mathrm{MHz}, \mathrm{CDCl}_{3}\right) \delta 176.4,140.2,128.4,127.5,125.9,71.5,66.3,30.4,28.6,17.9$; MS $m / z: 205\left(\mathrm{M}^{+}\right), 98(100 \%)$; HRMS (EI) calcd for $\mathrm{C}_{12} \mathrm{H}_{15} \mathrm{O}_{2} \mathrm{~N}\left(\mathrm{M}^{+}\right)$: 205.1103, found: 205.1098. 1-(2-Hydroxy-2-phenylethyl)pyrrolidin-2-one (5b): Colorless plates of $\mathrm{mp} \quad 116-117 \quad{ }^{\circ} \mathrm{C}$ (EtOAc/Hex); IR (neat) v 3362, $1663 \mathrm{~cm}^{-1} ;{ }^{1} \mathrm{H}$ NMR (400 MHz, $\left.\mathrm{CDCl}_{3}\right) \delta$ 7.41-7.26 (m, 5H), $4.98(\mathrm{t}$, $1 \mathrm{H}, J=5.1 \mathrm{~Hz}), 3.55(\mathrm{~d}, 2 \mathrm{H}, J=5.1 \mathrm{~Hz}), 3.35(\mathrm{~m}, 1 \mathrm{H}), 3.18(\mathrm{~m}, 1 \mathrm{H}), 2.42(\mathrm{t}, 2 \mathrm{H}, J=8.2 \mathrm{~Hz}), 2.06-1.90$ (m, 2H); ${ }^{13} \mathrm{C}$ NMR $\left(75 \mathrm{MHz}, \mathrm{CDCl}_{3}\right) \delta 177.3,142.0,128.4,127.7,125.7,73.3,52.1,49.6,30.8,18.4$; MS m/z: $205\left(\mathrm{M}^{+}\right), 99(100 \%)$; HRMS (EI) calcd for $\mathrm{C}_{12} \mathrm{H}_{15} \mathrm{O}_{2} \mathrm{~N}\left(\mathrm{M}^{+}\right)$: 205.1103, found: 205.1098.

$\left(R^{*}\right)$-5-(( $\left.R^{*}\right)$-(3,4-Methylenedioxyphenyl)(hydroxy)methyl)-1-methylpyrrolidin-2-one

$(3 c)$,

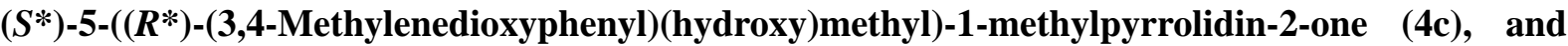
1-(2-(3,4-Methylenedioxyphenyl)-2-hydroxyethyl)pyrrolidin-2-one (5c): The title compounds were obtained according to the above-mentioned procedure from piperonal $(\mathbf{2 c})(450 \mathrm{mg}, 3.0 \mathrm{mmol})$, 1-methyl-2-pyrrolidinone (1) $(10 \mathrm{~mL}, 105 \mathrm{mmol})$ and $\mathrm{Et}_{3} \mathrm{~B}(2.6 \mathrm{~mL}, 18 \mathrm{mmol})$. Reaction time: $14 \mathrm{~h}$. Purification by silica gel column chromatography (EtOAc/Hex 1:4) gave ethyl adduct 6c (41 mg, 8\%) as a colorless oil. Further elution with $\mathrm{MeOH} / \mathrm{EtOAc}(1: 40)$ gave a mixture of alcohols $\mathbf{3 c}, \mathbf{4 c}$, and $\mathbf{5 c}$ (505 mg, 68\%) as a colorless solid. The yields and the ratio of the products were determined by ${ }^{1} \mathrm{H}$ NMR analysis to be 3c/4c (465 $\mathrm{mg}, 62 \%$, dr 77:23) and $\mathbf{5 c}(40 \mathrm{mg}, 6 \%)$. Recrystallization of the mixture from EtOAc/ $\mathrm{CH}_{2} \mathrm{Cl}_{2}$ gave $3 \mathbf{c}$ and $\mathbf{4 c}$, both as colorless plates. A pure sample of alcohol $\mathbf{5 c}$ was 
obtained as follows: acetylation of the residue obtained by concentration of the mother liquor that originated from recrystallization $\left(\mathrm{Ac}_{2} \mathrm{O}, \mathrm{Et}_{3} \mathrm{~N}, \mathrm{DMAP}, \mathrm{CH}_{2} \mathrm{Cl}_{2}\right.$, r.t.), chromatographic separation (MeOH/EtOAc 1:50), and hydrolysis of the acetate $\left(\mathrm{K}_{2} \mathrm{CO}_{3}\right.$, aq. $\mathrm{MeOH}$, r.t.). The spectroscopic data of alcohol $\mathbf{6 c}$ were identical to those reported (See reference 2 in S15). The configuration of major compound 3c was determined to be threo by X-ray crystallographic analysis of the 4-bromobenzoate derived from 3c (For crystallographic data, see CIF file). threo-Alcohol 3c: Mp 157-158 ${ }^{\circ} \mathrm{C}$ $\left(\mathrm{EtOAc} / \mathrm{CH}_{2} \mathrm{Cl}_{2}\right.$ ); IR (neat) v 3350, $1664 \mathrm{~cm}^{-1} ;{ }^{1} \mathrm{H}$ NMR (300 MHz, $\left.\mathrm{CDCl}_{3}\right) \delta 6.85-6.71(\mathrm{~m}, 3 \mathrm{H}), 5.95$ (s, 2H), $4.64(\mathrm{~d}, 1 \mathrm{H}, J=6.1 \mathrm{~Hz}), 3.72(\mathrm{ddd}, 1 \mathrm{H}, J=9.5,6.1,3.5 \mathrm{~Hz}), 2.96(\mathrm{~s}, 3 \mathrm{H}), 2.44(\mathrm{br}, 1 \mathrm{H})$, 2.14-1.66 (m, 4H); ${ }^{13} \mathrm{C}$ NMR (75 MHz, $\left.\mathrm{CDCl}_{3}\right) \oint 175.9,148.0,147.5,134.7,119.9,108.3,106.6,101.2$, 75.9, 65.4, 30.3, 29.7, 21.1; MS m/z: $249\left(\mathrm{M}^{+}\right), 98(100 \%)$; HRMS (EI) calcd for $\mathrm{C}_{13} \mathrm{H}_{15} \mathrm{O}_{4} \mathrm{~N}\left(\mathrm{M}^{+}\right)$: 249.1001, found: 249.1004. erythro-Alcohol 4c: $\mathrm{Mp} 143-144{ }^{\circ} \mathrm{C}\left(\mathrm{EtOAc} / \mathrm{CH}_{2} \mathrm{Cl}_{2}\right)$; IR (neat) v 3350, $1666 \mathrm{~cm}^{-1} ;{ }^{1} \mathrm{H}$ NMR (400 MHz, $\left.\mathrm{CDCl}_{3}\right) \delta$ 6.89-6.76 (m, 3H), $5.95(\mathrm{~s}, 2 \mathrm{H}), 4.96(\mathrm{~d}, 1 \mathrm{H}, J=3.0 \mathrm{~Hz})$, $3.65(\mathrm{dt}, 1 \mathrm{H}, J=8.8,3.0 \mathrm{~Hz}), 2.89$ (s, 3H), $2.36(\mathrm{~m}, 1 \mathrm{H}), 2.20(\mathrm{ddd}, 1 \mathrm{H}, J=17.0,10.5,4.4 \mathrm{~Hz}), 2.00$ (m, 1H), $1.74(\mathrm{~m}, 1 \mathrm{H}) ;{ }^{13} \mathrm{C}$ NMR $\left(100 \mathrm{MHz}, \mathrm{CDCl}_{3}\right) \oint 176.3,147.7,146.9,134.3,119.2,108.1,106.6$, 101.0, 71.4, 66.3, 30.3, 28.6, 18.1; MS m/z: $249\left(\mathrm{M}^{+}\right), 98(100 \%)$; HRMS (EI) calcd for $\mathrm{C}_{13} \mathrm{H}_{15} \mathrm{O}_{4} \mathrm{~N}$ $\begin{array}{llll}\left(\mathrm{M}^{+}\right): & 249.1001, & \text { found: } & 249.1001 \text {. }\end{array}$ 1-(2-(3,4-Methylenedioxyphenyl)-2-hydroxyethyl)pyrrolidin-2-one (5c): Colorless prisms of mp $170-171{ }^{\circ} \mathrm{C}\left(\mathrm{EtOAc} / \mathrm{CH}_{2} \mathrm{Cl}_{2}\right)$; IR (neat) v 3362, $1663 \mathrm{~cm}^{-1} ;{ }^{1} \mathrm{H}$ NMR $\left(300 \mathrm{MHz}, \mathrm{CDCl}_{3}\right) \delta$ 6.91-6.77 (m, 3H), 5.96 (s, 2H), 4.88 (dd, 1H, J=6.5, 4.4 Hz), 3.51-3.48 (m, 2H), 3.37 (ddd, 1H, $J=9.5,7.7,6.5$ $\mathrm{Hz}), 3.25(\mathrm{ddd}, 1 \mathrm{H}, J=9.5,7.7,6.5 \mathrm{~Hz}), 2.42(\mathrm{t}, 2 \mathrm{H}, J=7.7 \mathrm{~Hz}), 2.00(\mathrm{~m}, 2 \mathrm{H}) ;{ }^{13} \mathrm{C}$ NMR $(100 \mathrm{MHz}$, $\left.\mathrm{CDCl}_{3}\right) \delta 177.1,147.8,147.0,136.0,119.0,108.1,106.3,101.0,73.2,52.2,49.6,30.9,18.5 ; \mathrm{MS} m / z:$ $249\left(\mathrm{M}^{+}\right), 99$ (100\%); HRMS (EI) calcd for $\mathrm{C}_{13} \mathrm{H}_{15} \mathrm{O}_{4} \mathrm{~N}\left(\mathrm{M}^{+}\right)$: 249.1001, found: 249.1000 .

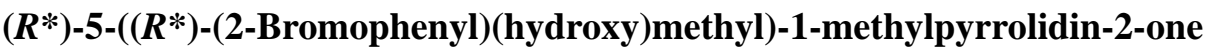
(3d), $\left(R^{*}\right)$-5-(( $\left.S^{*}\right)$-(2-Bromophenyl)(hydroxy)methyl)-1-methylpyrrolidin-2-one $(4 d)$, 1-(2-(2-Bromophenyl)-2-hydroxyethyl)pyrrolidin-2-one (5d): The title compounds were obtained according to the above-mentioned procedure from 2-bromobenzaldehyde (2d) (555 $\mathrm{mg}, 3.0 \mathrm{mmol}$ ), 1-methyl-2-pyrrolidinone (1) $(20 \mathrm{~mL}, 210 \mathrm{mmol})$ and $\mathrm{Et}_{3} \mathrm{~B}(2.6 \mathrm{~mL}, 18 \mathrm{mmol})$. Reaction time: $12 \mathrm{~h}$. 
Purification by silica gel column chromatography (EtOAc/Hex 1:3) gave ethyl adduct 6d (84 mg, 13\%) as a colorless oil and 2-bromobenzyl alcohol (7d) (17 $\mathrm{mg}, 3 \%)$. Further elution with $\mathrm{MeOH} /$ EtOAc (1:40) gave a mixture of alcohols $\mathbf{3 d}$, $\mathbf{4 d}$, and $\mathbf{5 d}(538 \mathrm{mg}, 63 \%)$ as a colorless solid. The yields and the ratio of the products were determined by ${ }^{1} \mathrm{H}$ NMR analysis to be $\mathbf{3 d} / \mathbf{4 d}$ (457 mg, $54 \%$, dr 42:58) and $\mathbf{5 d}(81 \mathrm{mg}, 9 \%)$. Recrystallization of the mixture from EtOAc/ $\mathrm{CH}_{2} \mathrm{Cl}_{2}$ gave $\mathbf{3 d}$ as colorless pillars and $\mathbf{4 d}$ as colorless plates. Pure compound $\mathbf{5 d}$ was obtained by recrystallization of the mother liquor from EtOAc/ $\mathrm{CH}_{2} \mathrm{Cl}_{2}$. Compound 7d was found to be identical to the commercially available material. Major product $\mathbf{4 d}$ was transformed into compound $\mathbf{4 b}$ via debromination with $\mathrm{Bu}_{3} \mathrm{SnH} / \mathrm{AIBN}$ (83\%), thereby confirming its erythro configuration. threo-Alcohol 3d: Mp 138-139 ${ }^{\circ} \mathrm{C}\left(\mathrm{EtOAc} / \mathrm{CH}_{2} \mathrm{Cl}_{2}\right)$; IR (neat) v 3350, $1666 \mathrm{~cm}^{-1} ;{ }^{1} \mathrm{H}$ NMR $\left(400 \mathrm{MHz}, \mathrm{CDCl}_{3}\right) \delta$ 7.57-7.53 (m, 2H), $7.35(\mathrm{~m}, 1 \mathrm{H}), 7.17(\mathrm{~m}, 1 \mathrm{H}), 5.11(\mathrm{dd}, 1 \mathrm{H}, J=6.0,5.0 \mathrm{~Hz}), 3.87$ (ddd, $1 \mathrm{H}, J=9.0,6.3,3.1 \mathrm{~Hz}), 3.23(\mathrm{br}$, 1H), $2.82(\mathrm{~s}, 3 \mathrm{H}), 2.49(\mathrm{dt}, 1 \mathrm{H}, J=17.0,9.0 \mathrm{~Hz}), 2.18(\mathrm{ddd}, 1 \mathrm{H}, J=17.0,10.0,3.7 \mathrm{~Hz}), 1.91(\mathrm{~m}, 1 \mathrm{H})$, $1.77(\mathrm{~m}, 1 \mathrm{H}) ;{ }^{13} \mathrm{C}$ NMR $\left(100 \mathrm{MHz}, \mathrm{CDCl}_{3}\right) \delta 176.2,140.8,132.8,129.5,128.5,127.9,122.6,76.1$, 65.2, 31.2, 30.2, 22.5; MS m/z: $283\left(\mathrm{M}^{+}\right), 98$ (100\%); HRMS (EI) calcd for $\mathrm{C}_{12} \mathrm{H}_{14} \mathrm{O}_{2} \mathrm{NBr}\left(\mathrm{M}^{+}\right)$: 283.0208, found: 283.0201. erythro-Alcohol 4d: $\mathrm{Mp} 218-219{ }^{\circ} \mathrm{C}\left(\mathrm{EtOAc} / \mathrm{CH}_{2} \mathrm{Cl}_{2}\right)$; IR (neat) v 3329, $1666 \mathrm{~cm}^{-1} ;{ }^{1} \mathrm{H}$ NMR $\left(300 \mathrm{MHz}, \mathrm{CDCl}_{3}\right) \delta 7.68(\mathrm{dd}, 1 \mathrm{H}, J=1.0,8.0 \mathrm{~Hz}), 7.53(\mathrm{dd}, 1 \mathrm{H}, J=1.0,8.0 \mathrm{~Hz})$, $7.38(\mathrm{dt}, 1 \mathrm{H}, J=1.0,8.0 \mathrm{~Hz}), 7.17$ (dt, 1H, $J=2.0,8.0 \mathrm{~Hz}), 5.42$ (d, 1H, $J=1.5 \mathrm{~Hz}), 3.90$ (ddd, 1H, $J=9.0$, 3.0, $1.5 \mathrm{~Hz}), 2.54(\mathrm{~m}, 1 \mathrm{H}), 2.25(\mathrm{ddd}, 1 \mathrm{H}, J=17.0,10.6,4.0 \mathrm{~Hz}), 2.10(\mathrm{~m}, 1 \mathrm{H}), 1.63(\mathrm{~m}, 1 \mathrm{H}) ;{ }^{13} \mathrm{C} \mathrm{NMR}$ $\left(75 \mathrm{MHz}, \mathrm{CDCl}_{3}\right) \delta 176.5,139.3,132.6,129.1,128.5,127.6,121.4,69.9,63.4,30.7,28.0,16.9 ; \mathrm{MS}$ m/z: $283\left(\mathrm{M}^{+}\right), 98(100 \%)$; HRMS (EI) calcd for $\mathrm{C}_{12} \mathrm{H}_{14} \mathrm{O}_{2} \mathrm{NBr}\left(\mathrm{M}^{+}\right)$: 283.0208, found: 283.0206 . 1-(2-(2-Bromophenyl)-2-hydroxyethyl)pyrrolidin-2-one (5d): Colorless prisms of mp 111-112 ${ }^{\circ} \mathrm{C}$ $\left(\right.$ EtOAc/ $\mathrm{CH}_{2} \mathrm{Cl}_{2}$ ); IR (neat) v 3340, $1666 \mathrm{~cm}^{-1} ;{ }^{1} \mathrm{H}$ NMR $\left(300 \mathrm{MHz}, \mathrm{CDCl}_{3}\right) \delta 7.67$ (dd, $1 \mathrm{H}, J=7.7,1.7$ $\mathrm{Hz}), 7.50(\mathrm{dd}, 1 \mathrm{H}, J=7.7,1.0 \mathrm{~Hz}), 7.35(\mathrm{dt}, 1 \mathrm{H}, J=7.7,1.0 \mathrm{~Hz}), 7.14(\mathrm{dt}, 1 \mathrm{H}, J=7.7,1.7 \mathrm{~Hz}), 5.24$ (dd, 1H, $J=6.9,2.4 \mathrm{~Hz}), 3.67$ (dd, 1H, $J=14.6,6.9 \mathrm{~Hz}), 3.56$ (dd, 1H, $J=14.6,2.4 \mathrm{~Hz}), 3.49$ (m, 1H), 3.08 (ddd, $1 \mathrm{H}, J=9.4,8.4,5.7 \mathrm{~Hz}), 2.51-2.33(\mathrm{~m}, 2 \mathrm{H}), 2.11-1.86(\mathrm{~m}, 2 \mathrm{H}) ;{ }^{13} \mathrm{C}$ NMR $\left(100 \mathrm{MHz}, \mathrm{CDCl}_{3}\right)$ $\delta 177.9,140.5,132.4,129.0,127.9,127.6,121.3,72.9,50.5,49.5,30.8,18.5 ; \mathrm{MS} m / z: 283\left(\mathrm{M}^{+}\right), 99$ (100\%); HRMS (EI) calcd for $\mathrm{C}_{12} \mathrm{H}_{14} \mathrm{O}_{2} \mathrm{NBr} \quad\left(\mathrm{M}^{+}\right)$: 283.0208, found: 283.0206. 
1-(2-bromophenyl)propan-1-ol (6d): IR (neat) $v 3350 \mathrm{~cm}^{-1} ;{ }^{1} \mathrm{H} \mathrm{NMR}\left(300 \mathrm{MHz}, \mathrm{CDCl}_{3}\right) \delta$ 7.56-7.50 (m, 2H), 7.33 (t, 1H, $J=7.5 \mathrm{~Hz}), 7.12$ (dt, 1H, $J=7.5,1.7 \mathrm{~Hz}$ ), 5.01 (dd, 1H, J=7.7, $4.7 \mathrm{~Hz}$ ), 2.0 (brs, 1H), 1.91-1.64 (m, 2H), $1.05(\mathrm{t}, 1 \mathrm{H}, J=7.4 \mathrm{~Hz}) ;{ }^{13} \mathrm{C} \mathrm{NMR}\left(75 \mathrm{MHz}, \mathrm{CDCl}_{3}\right) \delta 143.5,132.6,128.7$, 127.6, 127.4, 122.1, 74.2, 30.5, 10.1; MS m/z: $214\left(\mathrm{M}^{+}\right), 185$ (100\%); HRMS (EI) calcd for $\mathrm{C}_{9} \mathrm{H}_{11} \mathrm{BrO}$ $\left(\mathrm{M}^{+}\right)$: 213.9993, found: 213.9990 .

$\left(R^{*}\right)$-5-(( $\left.R^{*}\right)$-1-Hydroxytridecyl)-1-methylpyrrolidin-2-one

(3e),

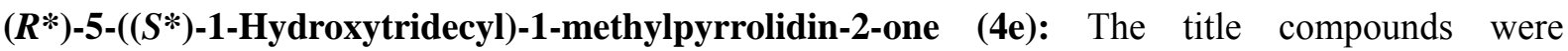
obtained according to the above-mentioned procedure from tridecanal (2e) (598 $\mathrm{mg}, 3.0 \mathrm{mmol}$ ), 1-methyl-2-pyrrolidinone (1) $(20 \mathrm{~mL}, 210 \mathrm{mmol})$ and $\mathrm{Et}_{3} \mathrm{~B}(2.6 \mathrm{~mL}, 18 \mathrm{mmol})$. Reaction time: $22 \mathrm{~h}$. Purification by silica gel column chromatography (MeOH/EtOAc 1:40) gave an inseparable mixture of alcohols $3 \mathbf{e}$ and $4 \mathbf{e}\left(447 \mathrm{mg}, 50 \%\right.$, dr 50:50) as a colorless solid. Product ratio was determined by ${ }^{1} \mathrm{H}$ NMR analysis. Pure samples of alcohols $3 \mathbf{e}$ and $\mathbf{4 e}$ were obtained as follows: acetylation of the mixture of alcohols $3 \mathbf{e}$ and $4 \mathbf{e}\left(\mathrm{Ac}_{2} \mathrm{O}, \mathrm{Et}_{3} \mathrm{~N}, \mathrm{DMAP}, \mathrm{CH}_{2} \mathrm{Cl}_{2}\right.$, r.t.), chromatographic separation $\left(\mathrm{MeOH} / \mathrm{CHCl}_{3}\right.$ 1:40), and hydrolysis of the acetates $\left(\mathrm{K}_{2} \mathrm{CO}_{3}\right.$, aq. $\mathrm{MeOH}$, r.t. $)$. Alcohol 3e, the carbinolic proton signal of which is upfield with respect to that of alcohol $\mathbf{4 e}$, has been tentatively assigned as a threo isomer. threo-Alcohol 3e: $\mathrm{Mp} 44-46{ }^{\circ} \mathrm{C}$; IR (neat) $\vee 3393,1668 \mathrm{~cm}^{-1}$; ${ }^{1} \mathrm{H}$ NMR (400 MHz, $\left.\mathrm{CDCl}_{3}\right) \delta 3.70(\mathrm{~m}, 1 \mathrm{H}), 3.53(\mathrm{ddd}, 1 \mathrm{H}, J=8.8,5.1,4.0 \mathrm{~Hz}), 2.89(\mathrm{~s}, 3 \mathrm{H}), 2.36(\mathrm{br}, 1 \mathrm{H}), 2.40$ (m, 1H), 2.26 (ddd, 1H, J=16.8, 10.2, $4.8 \mathrm{~Hz}), 2.25(\mathrm{~m}, 1 \mathrm{H}), 1.84(\mathrm{~m}, 1 \mathrm{H}), 1.42-1.22(\mathrm{~m}, 22 \mathrm{H}), 0.86(\mathrm{t}$, $3 \mathrm{H}, J=6.8 \mathrm{~Hz}) ;{ }^{13} \mathrm{C}$ NMR $\left(100 \mathrm{MHz}, \mathrm{CDCl}_{3}\right) \delta 175.8,73.6,64.5,32.4,31.9,30.17,30.12,29.7,29.63$, 29.62, 29.60, 29.3, 25.9, 22.7, 20.8, 14.1; MS m/z: $296\left(\mathrm{M}^{+}-1\right), 98$ (100\%); HRMS (FAB) calcd for $\mathrm{C}_{18} \mathrm{H}_{36} \mathrm{O}_{2} \mathrm{~N}\left(\mathrm{MH}^{+}\right)$: 298.2746 , found: 298.2743 . erythro-Alcohol 4e: $\mathrm{Mp} 70-71{ }^{\circ} \mathrm{C}$; IR (neat) $v$ 3391, $1678 \mathrm{~cm}^{-1} ;{ }^{1} \mathrm{H}$ NMR $\left(400 \mathrm{MHz}, \mathrm{CDCl}_{3}\right) \delta 3.90(\mathrm{~m}, 1 \mathrm{H}), 3.42(\mathrm{ddd}, 1 \mathrm{H}, J=8.6,4.2,1.3 \mathrm{~Hz}), 3.34(\mathrm{br}$, 1H), $2.81(\mathrm{~m}, 3 \mathrm{H}), 2.44$ (ddd, 1H, $J=17.1,10.5,7.1 \mathrm{~Hz}), 2.28$ (ddd, 1H, $J=16.4,10.5,5.7 \mathrm{~Hz}), 2.04$ (m, 1H), $1.90(\mathrm{~m}, 1 \mathrm{H}), 1.56-1.22(\mathrm{~m}, 22 \mathrm{H}), 0.87(\mathrm{t}, 3 \mathrm{H}, J=6.8 \mathrm{~Hz}) ;{ }^{13} \mathrm{C}$ NMR $\left(100 \mathrm{MHz}, \mathrm{CDCl}_{3}\right) \delta 176.0$, $69.0,65.0,32.8,31.9,30.7,29.7,29.69,29.67,29.62,29.60,29.4,28.0,26.3,22.7,17.5,14.2$; MS $m / z: 296\left(\mathrm{M}^{+}-1\right), 98(100 \%)$; HRMS (FAB) calcd for $\mathrm{C}_{18} \mathrm{H}_{36} \mathrm{O}_{2} \mathrm{~N}\left(\mathrm{MH}^{+}\right)$: 298.2746, found: 298.2748 . 
1-(2-Hydroxy-2-(4-methoxyphenyl)ethyl)-3-methylimidazolidin-2-one (17): The title compounds were obtained according to the above-mentioned procedure from 4-methoxybenzaldehyde (2a) (408 mg, $3.0 \mathrm{mmol})$, 1,3-dimethyl-2-imidazolidinone (8) (11 mL, $105 \mathrm{mmol})$ and $\mathrm{Et}_{3} \mathrm{~B}$ (2.6 mL, $\left.18 \mathrm{mmol}\right)$. After $10 \mathrm{~h}$, the mixture was partitioned between $\mathrm{Et}_{2} \mathrm{O}$ and $\mathrm{H}_{2} \mathrm{O}$. The separated aqueous phase was thoroughly extracted with $\mathrm{CH}_{2} \mathrm{Cl}_{2}$ and the combined organic extract was dried over $\mathrm{MgSO}_{4}$. Following solvent evaporation in vacuo, the residue was purified by silica gel column chromatography (EtOAc/Hex 1:4) to give ethyl adduct 6a (30 mg, 6\%) and further elution with MeOH/EtOAc (1:40) gave an inseparable mixture of alcohols threo-16, erythro-16, and $17(623 \mathrm{mg}, 83 \%)$ as a colorless solid. The yields and the ratio of the products were determined by ${ }^{1} \mathrm{H}$ NMR analysis to be threo-16/ erythro-16 (561 mg, 75\%, dr 62:38) and $\mathbf{1 7}$ (62 mg, 8\%). Pure samples of alcohols threo-16 and erythro-16 were obtained as follows: acetylation of the mixture of alcohols threo-16, erythro-16, and $17\left(\mathrm{Ac}_{2} \mathrm{O}, \mathrm{Et}_{3} \mathrm{~N}, \mathrm{DMAP}, \mathrm{CH}_{2} \mathrm{Cl}_{2}\right.$, r.t.), chromatographic separation (MeOH/EtOAc 1:40), and hydrolysis of the acetates $\left(\mathrm{K}_{2} \mathrm{CO}_{3}\right.$, aq. $\mathrm{MeOH}$, r.t.). Recrystallization from EtOAc/ $\mathrm{CH}_{2} \mathrm{Cl}_{2}$ gave erythro-16 as colorless pillars. The configuration of the minor compound erythro-16 was determined by X-ray crystallographic analysis (For crystallographic data, see CIF file). threo-Alcohol 16: colorless oil; IR (neat) v 3362, $1678 \mathrm{~cm}^{-1} ;{ }^{1} \mathrm{H}$ NMR $\left(300 \mathrm{MHz}, \mathrm{CDCl}_{3}\right)$ 8 7.29-7.24 (m, 2H), 6.93-6.87 (m, 2H), $4.66(\mathrm{~d}, 1 \mathrm{H}, J=6.5 \mathrm{~Hz}), 3.81(\mathrm{~s}, 3 \mathrm{H}), 3.69(\mathrm{ddd}, 1 \mathrm{H}, J=9.2,7.5,6.5 \mathrm{~Hz}), 3.05(\mathrm{t}, 1 \mathrm{H}, J=9.2$ $\mathrm{Hz}), 2.89(\mathrm{~s}, 3 \mathrm{H}), 2.84(\mathrm{dd}, 1 \mathrm{H}, J=9.2,7.7 \mathrm{~Hz}), 2.68(\mathrm{~s}, 3 \mathrm{H}) ;{ }^{13} \mathrm{C} \mathrm{NMR}\left(100 \mathrm{MHz}, \mathrm{CDCl}_{3}\right) \oint$ 161.9, 159.6, 132.7, 127.5, 114.1, 76.5, 61.3, 55.3, 47.9, 32.5, 31.1; MS m/z: $250\left(\mathrm{M}^{+}\right), 113(100 \%)$; HRMS (EI) calcd for $\mathrm{C}_{13} \mathrm{H}_{18} \mathrm{O}_{3} \mathrm{~N}_{2}\left(\mathrm{M}^{+}\right)$: 250.1317, found: 250.1319. erythro-Alcohol 16: Mp 135-136 ${ }^{\circ} \mathrm{C}$ $\left(\right.$ EtOAc $/ \mathrm{CH}_{2} \mathrm{Cl}_{2}$ ); IR (neat) v 3368, $1682 \mathrm{~cm}^{-1} ;{ }^{1} \mathrm{H}$ NMR (400 MHz, $\left.\mathrm{CDCl}_{3}\right) \delta$ 7.31-7.26 (m, 2H), 6.91-6.86 (m, 2H), 4.93 (d, 1H, J=3.3 Hz), 3.80 (s, 3H), 3.56 (ddd, 1H, J=9.0, 8.1, 3.3 Hz), 3.27 (dd, $1 \mathrm{H}, J=9.0,8.1 \mathrm{~Hz}), 2.98(\mathrm{t}, 1 \mathrm{H}, J=9.0 \mathrm{~Hz}), 2.75(\mathrm{~s}, 3 \mathrm{H}), 2.73(\mathrm{~s}, 3 \mathrm{H}) ;{ }^{13} \mathrm{C} \mathrm{NMR}\left(100 \mathrm{MHz}, \mathrm{CDCl}_{3}\right)$ $\delta 161.8,159.0,131.5,126.9,113.8,69.6,62.0,55.3,45.0,30.9,30.3 ; \mathrm{MS} m / z: 250\left(\mathrm{M}^{+}\right), 113(100 \%)$; HRMS (EI) calcd for $\mathrm{C}_{13} \mathrm{H}_{18} \mathrm{O}_{3} \mathrm{~N}_{2}\left(\mathrm{M}^{+}\right)$: 250.1317, found: 250.1315. Alcohol 17: IR (neat) $\vee$ 3410, $1670 \mathrm{~cm}^{-1} ;{ }^{1} \mathrm{H}$ NMR $\left(300 \mathrm{MHz}, \mathrm{CDCl}_{3}\right) \delta$ 7.22-7.16 (m, 2H), 6.90-6.84 (m, 2H), $4.66(\mathrm{dd}, 1 \mathrm{H}, J=8.9$, 
$4.1 \mathrm{~Hz}), 4.12(\mathrm{dd}, 1 \mathrm{H}, J=11.7,8.9 \mathrm{~Hz}), 3.93(\mathrm{dd}, 1 \mathrm{H}, J=11.7,4.1 \mathrm{~Hz}), 3.79$ (s, 3H), 3.32-3.19 (m, 3H), $3.05(\mathrm{~m}, 1 \mathrm{H}), 2.81(\mathrm{~s}, 3 \mathrm{H}) ;{ }^{13} \mathrm{C} \mathrm{NMR}\left(75 \mathrm{MHz}, \mathrm{CDCl}_{3}\right) \delta 190.5,159.2,129.5,128.7,114.1,63.8,61.0$, 55.3, 45.4, 41.2, 31.4; MS m/z: $232\left(\mathrm{M}^{+}-18\right), 219$ (100\%); HRMS (FAB) calcd for $\mathrm{C}_{13} \mathrm{H}_{19} \mathrm{O}_{3} \mathrm{~N}_{2}\left(\mathrm{MH}^{+}\right)$: 251.1396, found: 251.1394 .

$\left(R^{*}\right)$-Tetrahydro-6-(( $\left.R^{*}\right)$-hydroxy(4-methoxyphenyl)methyl)-1,3-dimethylpyrimidin-2(1H)-one (threo-18),

$\left(R^{*}\right)$-Tetrahydro-6-(( $\left.S^{*}\right)$-hydroxy(4-methoxyphenyl)methyl)-1,3-dimethylpyrimidin-2(1H)-one (erythro-18), Tetrahydro-3-(2-hydroxy-2-(4-methoxyphenyl)ethyl)-1-methylpyrimidin-2(1H)-one (19): The title compounds were obtained according to the above-mentioned procedure from 4-methoxybenzaldehyde (2a) (408 mg, 3.0 mmol), 1,3-dimethyl-2-pyrimidinone (9) (12.6 mL, 105 mmol) and $\mathrm{Et}_{3} \mathrm{~B}(2.6 \mathrm{~mL}, 18 \mathrm{mmol})$. After $16 \mathrm{~h}$, the mixture was partitioned between $\mathrm{Et}_{2} \mathrm{O}$ and $\mathrm{H}_{2} \mathrm{O}$. The separated aqueous phase was thoroughly extracted with $\mathrm{CH}_{2} \mathrm{Cl}_{2}$ and the combined organic extract was dried over $\mathrm{MgSO}_{4}$. Following solvent evaporation in vacuo, the residue was purified by silica gel column chromatography (EtOAc/Hex 1:4) to give ethyl adduct 6a (30 mg, 6\%) and 4-methoxybenzylalcohol (7a) (7 mg, 2\%). Further elution with MeOH/EtOAc (1:40) gave a mixture of alcohols threo-18, erythro-18, and $\mathbf{1 9}$ as a colorless oil. The yields and the ratio of the products were determined by ${ }^{1} \mathrm{H}$ NMR analysis of acetates $(761 \mathrm{mg}, 83 \%$ combined yield) derived from the mixture to be threo-18/erythro-18 (as acetates, $659 \mathrm{mg}, 72 \%$, dr 79:21) and $\mathbf{1 9}$ (as acetate, $102 \mathrm{mg}, 11 \%$ ). Pure samples of alcohols threo-18, erythro-18, and 19 were obtained by chromatographic separation (MeOH/EtOAc 1:50) and hydrolysis of the acetates $\left(\mathrm{K}_{2} \mathrm{CO}_{3}\right.$, aq. $\mathrm{MeOH}$, r.t.). Recrystallization from EtOAc/ $\mathrm{CH}_{2} \mathrm{Cl}_{2}$ gave erythro-18 as colorless plates and alcohol $\mathbf{1 9}$ as colorless pillars. The configuration of the minor compound erythro-18 was determined by X-ray crystallographic analysis (For crystallographic data, see CIF file). threo-Alcohol 18: colorless oil; IR (neat) v 3339, $1612 \mathrm{~cm}^{-1}$; ${ }^{1} \mathrm{H}$ NMR (300 MHz, $\left.\mathrm{CDCl}_{3}\right)$ 8 7.27-7.21 (m, 2H), 6.90-6.85 (m, 2H), 4.72 (d, 1H, J=8.0 Hz), 3.79 (s, 3H), $3.41(\mathrm{~m}, 1 \mathrm{H}), 3.13(\mathrm{~s}, 3 \mathrm{H}), 3.10(\mathrm{~m}, 1 \mathrm{H}), 2.91(\mathrm{~m}, 1 \mathrm{H}), 2.87(\mathrm{~s}, 3 \mathrm{H}), 1.84(\mathrm{~m}, 1 \mathrm{H}), 1.50(\mathrm{~m}, 1 \mathrm{H})$; ${ }^{13} \mathrm{C}$ NMR $\left(100 \mathrm{MHz}, \mathrm{CDCl}_{3}\right) \delta 159.2,155.9,133.8,127.4,113.7,74.9,62.8,55.1,44.7,37.6,35.4$, 22.2; MS m/z: $264\left(\mathrm{M}^{+}\right), 127(100 \%)$; HRMS (EI) calcd for $\mathrm{C}_{14} \mathrm{H}_{20} \mathrm{O}_{3} \mathrm{~N}_{2}\left(\mathrm{M}^{+}\right)$: 264.1474, found: 
264.1473. erythro-Alcohol 18: $\mathrm{Mp} 160-161{ }^{\circ} \mathrm{C}\left(\mathrm{EtOAc} / \mathrm{CH}_{2} \mathrm{Cl}_{2}\right)$; IR (neat) $v 3329,1612 \mathrm{~cm}^{-1} ;{ }^{1} \mathrm{H}$ NMR (300 MHz, $\left.\mathrm{CDCl}_{3}\right) \delta$ 7.30-7.25 (m, 2H), 6.92-6.86 (m, 2H), $4.82(\mathrm{~d}, 1 \mathrm{H}, J=6.0 \mathrm{~Hz}), 3.81(\mathrm{~s}, 3 \mathrm{H})$, $3.51(\mathrm{ddd}, 1 \mathrm{H}, J=11.5,11.5,5.0 \mathrm{~Hz}), 3.33(\mathrm{ddd}, 1 \mathrm{H}, J=6.0,6.0,3.0 \mathrm{~Hz}), 3.10$ (ddd, $1 \mathrm{H}, J=11.5,6.0$, $3.0 \mathrm{~Hz}), 2.94(\mathrm{~s}, 3 \mathrm{H}), 2.62(\mathrm{~s}, 3 \mathrm{H}), 2.11(\mathrm{~m}, 1 \mathrm{H}), 1.82(\mathrm{~m}, 1 \mathrm{H}) ;{ }^{13} \mathrm{C} \mathrm{NMR}\left(100 \mathrm{MHz}, \mathrm{CDCl}_{3}\right) \delta 159.2$, 156.5, 133.5, 127.4, 113.9, 72.7, 63.2, 55.3, 45.3, 35.8, 35.6, 21.9; MS m/z: $264\left(\mathrm{M}^{+}\right), 127(100 \%)$; HRMS (EI) calcd for $\mathrm{C}_{14} \mathrm{H}_{20} \mathrm{O}_{3} \mathrm{~N}_{2}\left(\mathrm{M}^{+}\right)$: 264.1474, found: 264.1473. Alcohol 19: Mp 99-100 ${ }^{\circ} \mathrm{C}$ $\left(\mathrm{EtOAc} / \mathrm{CH}_{2} \mathrm{Cl}_{2}\right.$ ); IR (neat) v 3371, $1610 \mathrm{~cm}^{-1} ;{ }^{1} \mathrm{H}$ NMR (300 MHz, $\left.\mathrm{CDCl}_{3}\right) \delta$ 7.33-7.28 (m, 2H), 6.90-6.84 (m, 2H), 4.87 (dd, 1H, J=7.0, 3.0 Hz), 3.80 (s, 3H), 3.56 (dd, 1H, J=15.0, 7.0 Hz), 3.47 (dd, $1 \mathrm{H}, J=15.0,3.0 \mathrm{~Hz}), 3.27-3.18(\mathrm{~m}, 3 \mathrm{H}), 2.96(\mathrm{~s}, 3 \mathrm{H}), 2.95-2.88(\mathrm{~m}, 1 \mathrm{H}), 1.91-1.80(\mathrm{~m}, 2 \mathrm{H}) ;{ }^{13} \mathrm{C} \mathrm{NMR}$ $\left(75 \mathrm{MHz}, \mathrm{CDCl}_{3}\right) \delta 158.9,158.5,135.1,127.0,113.6,73.7,58.1,55.3,48.3,47.6,35.8,22.2 ; \mathrm{MS} m / z$ : $264\left(\mathrm{M}^{+}\right), 128(100 \%)$; HRMS (EI) calcd for $\mathrm{C}_{14} \mathrm{H}_{20} \mathrm{O}_{3} \mathrm{~N}_{2}\left(\mathrm{M}^{+}\right)$: 264.1474, found: 264.1474.

$\left(R^{*}\right)$-(4-Methoxyphenyl)(( $\left.R^{*}\right)$-1-methylpyrrolidin-2-yl)methanol

(threo-20), $\left(R^{*}\right)$-(4-Methoxyphenyl)((S*)-1-methylpyrrolidin-2-yl)methanol (erythro-20), 1-(4-Methoxyphenyl)-2-(pyrrolidin-1-yl)ethanol (21): The title compounds were obtained according to the above-mentioned procedure from 4-methoxybenzaldehyde (2a) (408 $\mathrm{mg}, 3.0 \mathrm{mmol}$ ), 1-methylpyrrolidine (10) $(11 \mathrm{~mL}, 105 \mathrm{mmol})$ and $\mathrm{Et}_{3} \mathrm{~B}(2.6 \mathrm{~mL}, 18 \mathrm{mmol})$. After $36 \mathrm{~h}, 28 \% \mathrm{NH}_{4} \mathrm{OH}$ was added and the mixture was extracted with $\mathrm{CH}_{2} \mathrm{Cl}_{2}$. The organic extract was dried over $\mathrm{Na}_{2} \mathrm{SO}_{4}$ and concentrated in vacuo. Purification by silica gel column chromatography $\left(\mathrm{MeOH} / \mathrm{EtOAc} / 28 \% \mathrm{NH}_{4} \mathrm{OH}\right.$ 1:30:1) gave a mixture of alcohols threo-20, erythro-20, and 21 (559 $\mathrm{mg}, 84 \%)$ as a colorless solid. The yields and the ratio of the products were determined by ${ }^{1} \mathrm{H}$ NMR analysis to be threo-20/erythro-20 (525 mg, 79\%, dr 58:42) and $\mathbf{2 1}$ (34 mg, 5\%). The configuration of major alcohol threo-20 was determined by chemical correlation with threo amine derived from amide 3a whose configuration was unambiguously determined by X-ray crystallographic analysis (see page S2); the reduction of 3a with LAH in 1,4-dioxane afforded threo-20 in 86\% yield. threo-Alcohol 20: Mp 70-74 ${ }^{\circ} \mathrm{C}$; IR (neat) v $3383 \mathrm{~cm}^{-1} ;{ }^{1} \mathrm{H}$ NMR $\left(300 \mathrm{MHz}, \mathrm{CDCl}_{3}\right) \delta$ 7.31-7.25 (m, 2H), 6.89-6.84 (m, 2H), 4.28 (d, 1H, $J=5.5 \mathrm{~Hz}), 3.80(\mathrm{~s}, 3 \mathrm{H}), 3.14-3.06(\mathrm{~m}, 1 \mathrm{H}), 2.71(\mathrm{~m}, 1 \mathrm{H}), 2.37(\mathrm{~m}, 1 \mathrm{H}), 2.28(\mathrm{~s}, 3 \mathrm{H}), 1.93-1.59$ (m, 4H); ${ }^{13} \mathrm{C}$ NMR $\left(75 \mathrm{MHz}, \mathrm{CDCl}_{3}\right) \delta 158.7,135.8,127.3,113.6,75.3,71.7,57.6,55.2,44.1,29.3$, 
24.4; MS m/z: $220\left(\mathrm{M}^{+}-1\right), 84(100 \%)$; HRMS (FAB) calcd for $\mathrm{C}_{13} \mathrm{H}_{20} \mathrm{O}_{2} \mathrm{~N}\left(\mathrm{MH}^{+}\right)$: 222.1494, found: 222.1493. erythro-Alcohol 20: IR (neat) $v 3425 \mathrm{~cm}^{-1} ;{ }^{1} \mathrm{H}$ NMR $\left(400 \mathrm{MHz}, \mathrm{CDCl}_{3}\right) \delta$ 7.30-7.26 (m, 2H), 6.89-6.85 (m, 2H), $4.82(\mathrm{~d}, 1 \mathrm{H}, J=2.9 \mathrm{~Hz}), 3.80(\mathrm{~s}, 3 \mathrm{H}), 3.14(\mathrm{~m}, 1 \mathrm{H}), 2.47(\mathrm{~m}, 1 \mathrm{H}), 2.45(\mathrm{~s}, 3 \mathrm{H})$, $2.33(\mathrm{dt}, 1 \mathrm{H}, J=9.3,7.6 \mathrm{~Hz}), 1.78-1.56(\mathrm{~m}, 3 \mathrm{H}), 1.30(\mathrm{~m}, 1 \mathrm{H}) ;{ }^{13} \mathrm{C} \mathrm{NMR}\left(100 \mathrm{MHz}, \mathrm{CDCl}_{3}\right) \delta 158.4$, 133.7, 126.5, 113.5, 71.0, 69.4, 57.6, 55.2, 40.0, 23.9, 22.9; MS m/z: $221\left(\mathrm{M}^{+}\right), 84(100 \%)$; HRMS (FAB) calcd for $\mathrm{C}_{13} \mathrm{H}_{20} \mathrm{O}_{2} \mathrm{~N}\left(\mathrm{MH}^{+}\right): 222.1494$, found: 222.1501. Alcohol 21: Mp 82-83 ${ }^{\circ} \mathrm{C}$; IR (neat) $v$ $3150 \mathrm{~cm}^{-1} ;{ }^{1} \mathrm{H}$ NMR $\left(300 \mathrm{MHz}, \mathrm{CDCl}_{3}\right) \delta$ 7.33-7.26 (m, 2H), 6.91-6.85 (m, 2H), $4.65(\mathrm{dd}, 1 \mathrm{H}, J=10.5$, $3.2 \mathrm{~Hz}) 3.80(\mathrm{~s}, 3 \mathrm{H}), 2.80-2.70(\mathrm{~m}, 3 \mathrm{H}), 2.58-2.48(\mathrm{~m}, 2 \mathrm{H}), 2.44$ (dd, 1H, J=12.0, $3.2 \mathrm{~Hz}), 1.86-1.71$ $(\mathrm{m}, 4 \mathrm{H}) ;{ }^{13} \mathrm{C}$ NMR $\left(75 \mathrm{MHz}, \mathrm{CDCl}_{3}\right) \delta 159.0,134.5,127.1,113.7,70.3,64.1,55.2,53.8,23.7$; MS $m / z: 221\left(\mathrm{M}^{+}\right), 84(100 \%)$; HRMS (EI) calcd for $\mathrm{C}_{13} \mathrm{H}_{19} \mathrm{O}_{2} \mathrm{~N}\left(\mathrm{M}^{+}\right)$: 221.1416, found: 221.1410.

1-(Diethylamino)-1-(4-methoxyphenyl)propan-1-ol (22): The title compound was obtained according to the above-mentioned procedure from 4-methoxybenzaldehyde (2a) (408 $\mathrm{mg}, 3.0 \mathrm{mmol}$ ), triethylamine (11) $(14.6 \mathrm{~mL}, 105 \mathrm{mmol})$ and $\mathrm{Et}_{3} \mathrm{~B}(2.6 \mathrm{~mL}, 18 \mathrm{mmol})$. Flow rate of air: 35-40 $\mathrm{mL} \cdot \mathrm{h}^{-1} \cdot \mathrm{mmol}$ aldehyde ${ }^{-1}$. After $16 \mathrm{~h}, 28 \% \mathrm{NH}_{4} \mathrm{OH}$ was added and the mixture was extracted with $\mathrm{CH}_{2} \mathrm{Cl}_{2}$. The organic extract was dried over $\mathrm{Na}_{2} \mathrm{SO}_{4}$ and concentrated in vacuo. Purification by silica gel column chromatography $\left(\mathrm{MeOH} / \mathrm{EtOAc} / 28 \% \mathrm{NH}_{4} \mathrm{OH}\right.$ 2:20:1) gave less polar alcohol major-22 (390 mg, 55\%) and more polar alcohol minor-22 (283 mg, 40\%), both as a yellow oil. The configuration of the products was not determined. Major-22: IR (neat) $\vee 3329 \mathrm{~cm}^{-1} ;{ }^{1} \mathrm{H}$ NMR (300 $\left.\mathrm{MHz}, \mathrm{CDCl}_{3}\right) \delta$ 7.30-7.24 (m, 2H), 6.89-6.83 (m, 2H), $4.15(\mathrm{~d}, 1 \mathrm{H}, J=9.7 \mathrm{~Hz}) 3.80(\mathrm{~s}, 3 \mathrm{H}), 2.75-2.63$ (m, 3H), 2.39 (dq, 2H, $J=13.4,6.9 \mathrm{~Hz}), 1.12(\mathrm{t}, 6 \mathrm{H}, J=6.9 \mathrm{~Hz}), 0.74(\mathrm{~d}, 3 \mathrm{H}, J=6.6 \mathrm{~Hz}) ;{ }^{13} \mathrm{C}$ NMR $(75$ $\left.\mathrm{MHz}, \mathrm{CDCl}_{3}\right) \delta 159.1,134.6,128.3,113.6,74.0,62.3,55.2,43.1,14.3,8.4 ; \mathrm{MS} m / z: 236\left(\mathrm{M}^{+}-1\right), 100$ (100\%); HRMS (FAB) calcd for $\mathrm{C}_{14} \mathrm{H}_{24} \mathrm{O}_{2} \mathrm{~N}\left(\mathrm{MH}^{+}\right)$: 238.1807, found: 238.1806. Minor-22: IR (neat) v $3404 \mathrm{~cm}^{-1} ;{ }^{1} \mathrm{H}$ NMR $\left(300 \mathrm{MHz}, \mathrm{CDCl}_{3}\right) \delta$ 7.24-7.20 (m, 2H), 6.88-6.83 (m, 2H), $4.64(\mathrm{~d}, 1 \mathrm{H}, J=4.8$ Hz) 3.80 (s, 3H), 2.99 (dq, 1H, J=7.0, $4.8 \mathrm{~Hz}), 2.48$ (q, 4H, J=7.0 Hz), 1.01 (t, 6H, J=7.0 Hz), 0.89 (d, $3 \mathrm{H}, J=7.0 \mathrm{~Hz}) ;{ }^{13} \mathrm{C} \mathrm{NMR}\left(75 \mathrm{MHz}, \mathrm{CDCl}_{3}\right) \delta 158.5,134.5,127.2,113.1,72.5,60.1,55.2,44.0,13.1$, 10.5; MS m/z: $236\left(\mathrm{M}^{+}-1\right), 100(100 \%)$; HRMS (FAB) calcd for $\mathrm{C}_{14} \mathrm{H}_{24} \mathrm{O}_{2} \mathrm{~N}\left(\mathrm{MH}^{+}\right): 238.1807$, found: 238.1801. 
2-(N-ethyl- $N$-phenylamino)-1-(4-methoxyphenyl)propan-1-ol (23): The title compound was obtained according to the above-mentioned procedure from 4-methoxybenzaldehyde (2a) (134 mg, 1.0 mmol), $N, N$-diethylaniline $(\mathbf{1 2})(5.6 \mathrm{~mL}, 35 \mathrm{mmol})$ and $\mathrm{Et}_{3} \mathrm{~B}(0.87 \mathrm{~mL}, 6 \mathrm{mmol})$. Flow rate of air: 35-40 $\mathrm{mL} \cdot \mathrm{h}^{-1} \cdot \mathrm{mmol}$ aldehyde ${ }^{-1}$. After $21 \mathrm{~h}$, sat. $\mathrm{NH}_{4} \mathrm{Cl}$ solution was added and the mixture was extracted with $\mathrm{CH}_{2} \mathrm{Cl}_{2}$. The organic extract was dried over $\mathrm{Na}_{2} \mathrm{SO}_{4}$ and concentrated in vacuo. Purification by silica gel column chromatography (EtOAc/Hex 1:4) gave a mixture of alcohols major-23 and minor-23 (185 mg, 65\%, dr 83:17) as a colorless oil. Ethyl adduct 6a (11 mg, 7\%) and reduction product $7 \mathbf{a}(13 \mathrm{mg}, 9 \%)$ were also obtained as a colorless oil. The data shown below are those of pure alcohols major-23 and minor-23, which were obtained by careful purification via flash silica gel chromatography (EtOAc/ $\mathrm{CH}_{2} \mathrm{Cl}_{2}$ 1:12). The configuration of the products was not determined. Major-23: IR (neat) $v 3447 \mathrm{~cm}^{-1} ;{ }^{1} \mathrm{H}$ NMR (300 MHz, $\left.\mathrm{CDCl}_{3}\right) \delta 7.39-7.26$ (m, 4H), 7.07 (d, 2H, J=8.2 Hz), 6.95-6.89 (m, 3H), 4.48 (d, 1H, J=9.5 Hz), 3.93 (brs, 1H), 3.82 (s, 3H), 3.64 (dq, $1 \mathrm{H}, J=9.5,6.8 \mathrm{~Hz}), 3.27$ (q, 2H, $J=7.0 \mathrm{~Hz}), 1.22(\mathrm{t}, 3 \mathrm{H}, J=7.0 \mathrm{~Hz}), 0.85(\mathrm{~d}, 3 \mathrm{H}, J=6.8 \mathrm{~Hz}) ;{ }^{13} \mathrm{C} \mathrm{NMR}$ $\left(75 \mathrm{MHz}, \mathrm{CDCl}_{3}\right) \delta 159.4,149.2,133.4,129.1,128.4,120.3,118.9,113.8,75.1,65.6,55.2,38.5,14.1$, 11.8; MS m/z: $285\left(\mathrm{M}^{+}\right), 148$ (100\%); HRMS (EI) calcd for $\mathrm{C}_{18} \mathrm{H}_{23} \mathrm{NO}_{2}\left(\mathrm{M}^{+}\right)$: 285.1729, found: 285.1722. Minor-23: IR (neat) $v 3416 \mathrm{~cm}^{-1} ;{ }^{1} \mathrm{H}$ NMR $\left(300 \mathrm{MHz}, \mathrm{CDCl}_{3}\right) \delta$ 7.32-7.20 (m, 4H), 6.90-6.70 (m, 5H), 4.87 (d, 1H, J=4.9 Hz) 3.97 (m, 1H), 3.80 (s, 3H), 3.38 (dq, 1H, J=21.6, $7.0 \mathrm{~Hz})$, $3.24(\mathrm{dq}, 1 \mathrm{H}, J=21.6,7.0 \mathrm{~Hz}), 2.13$ (brs, $1 \mathrm{H}), 1.23$ (d, 3H, $J=6.8 \mathrm{~Hz}), 1.07$ (t, 3H, $J=7.0 \mathrm{~Hz}) ;{ }^{13} \mathrm{C}$ NMR $\left(75 \mathrm{MHz}, \mathrm{CDCl}_{3}\right) \delta 158.9,148.3,134.8,129.1,127.2,117.3,115.2,113.6,75.2,60.1,55.2,40.1,13.8$, 12.5; MS m/z: $285\left(\mathrm{M}^{+}\right), 148(100 \%)$; HRMS (EI) calcd for $\mathrm{C}_{18} \mathrm{H}_{23} \mathrm{NO}_{2}\left(\mathrm{M}^{+}\right)$: 285.1729, found: 285.1728.

\section{(4-Methoxyphenyl)(4-methylmorpholin-3-yl)methanol (24),}

1-(4-Methoxyphenyl)-2-morpholinoethanol (25): The title compounds were obtained according to the above-mentioned procedure from 4-methoxybenzaldehyde (2a) (408 $\mathrm{mg}, 3.0 \mathrm{mmol}$ ), 4-methylmorpholine (13) (11.5 mL, $105 \mathrm{mmol})$ and $\mathrm{Et}_{3} \mathrm{~B}$ (2.6 mL, $\left.18 \mathrm{mmol}\right)$. After $36 \mathrm{~h}, 28 \% \mathrm{NH}_{4} \mathrm{OH}$ was added and the mixture was extracted with $\mathrm{CH}_{2} \mathrm{Cl}_{2}$. The organic extract was dried over $\mathrm{Na}_{2} \mathrm{SO}_{4}$ and concentrated in vacuo. Purification by silica gel column chromatography (EtOAc/Hex 1:4) gave ethyl 
adduct 6a (30 mg, 6\%). Further elution with $\mathrm{MeOH} / \mathrm{EtOAc} / 28 \% \mathrm{NH}_{4} \mathrm{OH}(1: 30: 1)$ gave a mixture of alcohols major-24, minor-24, and $\mathbf{2 5}$ (481mg, 68\%) as a colorless solid. The yields and the ratio of the products were determined by ${ }^{1} \mathrm{H}$ NMR analysis to be major-24/minor-24 (404 mg, 57\%, $\mathrm{dr} 52: 48$ ) and 25 (77 mg, 11\%). Recrystallization of the mixture from EtOAc/Hex gave major-24 as colorless solids and minor-24 as colorless pillars. The configuration of the products was not determined. Major-24: Mp 69-70 ${ }^{\circ} \mathrm{C}$ (EtOAc/Hex); IR (neat) v $3416 \mathrm{~cm}^{-1} ;{ }^{1} \mathrm{H}$ NMR (300 MHz, $\left.\mathrm{CDCl}_{3}\right) \delta$ 7.34-7.28 (m, 2H), 6.90-6.84 (m, 2H), 4.76 (d, 1H, J=7.0 Hz), 3.80 (s, 3H), 3.79-3.72 (m, 1H), 3.68 (dd, 1H, J=12.0, 3.5 Hz), 3.53 (ddd, 1H, $J=12.0,5.0,3.5 \mathrm{~Hz}), 3.35$ (dd, 1H, $J=12.0,4.5 \mathrm{~Hz}), 3.17$ (ddd, 1H, $J=13.4,8.6,3.5$ $\mathrm{Hz}), 2.51(\mathrm{~s}, 3 \mathrm{H}), 2.50-2.44(\mathrm{~m}, 1 \mathrm{H}), 2.39(\mathrm{dt}, 1 \mathrm{H}, J=7.0,4.0 \mathrm{~Hz}),{ }^{13} \mathrm{C}$ NMR $\left(100 \mathrm{MHz}, \mathrm{CDCl}_{3}\right) \delta$ 159.0, 134.4, 127.7, 113.7, 69.6, 65.8, 62.9, 62.6, 55.2, 49.8, 42.6; MS m/z: $237\left(\mathrm{M}^{+}\right), 100$ (100\%); HRMS (EI) calcd for $\mathrm{C}_{13} \mathrm{H}_{19} \mathrm{O}_{3} \mathrm{~N}\left(\mathrm{M}^{+}\right)$: 237.1365, found: 237.1367. Minor-24: Mp 63-64 ${ }^{\circ} \mathrm{C}$ (EtOAc/Hex); IR (neat) v $3425 \mathrm{~cm}^{-1} ;{ }^{1} \mathrm{H}$ NMR $\left(300 \mathrm{MHz}, \mathrm{CDCl}_{3}\right) \delta$ 7.25-7.21 (m, 2H), 6.90-6.85 (m, 2H), $5.12(\mathrm{~d}, 1 \mathrm{H}, J=3.0 \mathrm{~Hz}), 3.80(\mathrm{~s}, 3 \mathrm{H}), 3.74(\mathrm{dt}, 1 \mathrm{H}, J=11.0,2.0 \mathrm{~Hz}), 3.59-3.49$ (m, 2H), 3.39 (t, 1H, $J=11.0 \mathrm{~Hz}), 3.19$ (dd, 1H, $J=12.0,4.0 \mathrm{~Hz}), 2.77$ (d, 1H, $J=11.5 \mathrm{~Hz}), 2.57-2.46(\mathrm{~m}, 1 \mathrm{H}), 2.50(\mathrm{~s}, 3 \mathrm{H})$, 2.38-2.30 (m, 1H); ${ }^{13} \mathrm{C}$ NMR (75 MHz, $\left.\mathrm{CDCl}_{3}\right) \delta 158.7,131.5,126.4,113.7,68.6,66.5,66.2,65.5$, 55.2, 55.1, 42.6; MS m/z: $237\left(\mathrm{M}^{+}\right), 100$ (100\%); HRMS (EI) calcd for $\mathrm{C}_{13} \mathrm{H}_{19} \mathrm{O}_{3} \mathrm{~N}\left(\mathrm{M}^{+}\right): 237.1365$, found: 237.1361. Alcohol 25: Mp 82-83 ${ }^{\circ} \mathrm{C}$; IR (neat) $v 3435 \mathrm{~cm}^{-1} ;{ }^{1} \mathrm{H}$ NMR $\left(300 \mathrm{MHz}, \mathrm{CDCl}_{3}\right) \delta$ 7.31-7.26 (m, 2H), 6.91-6.85 (m, 2H), $4.71(\mathrm{dd}, 1 \mathrm{H}, J=8.5,5.0 \mathrm{~Hz}), 3.80(\mathrm{~s}, 3 \mathrm{H}), 3.77-3.73(\mathrm{~m}, 4 \mathrm{H})$, 2.77-2.70 (m, 2H), 2.51-2.42 (m, 4H); ${ }^{13} \mathrm{C}$ NMR $\left(75 \mathrm{MHz}, \mathrm{CDCl}_{3}\right) \delta 159.1,133.9,127.1,113.8,68.2$, 67.0, 66.7, 55.3, 53.5; MS m/z: $237\left(\mathrm{M}^{+}\right), 100$ (100\%); HRMS (EI) calcd for $\mathrm{C}_{13} \mathrm{H}_{19} \mathrm{O}_{3} \mathrm{~N}^{(}\left(\mathrm{M}^{+}\right)$: 237.1365, found: 237.1364 .

$\left(\boldsymbol{R}^{*}\right)-4-\left(\left(\boldsymbol{R}^{*}\right)\right.$-Hydroxy(4-methoxyphenyl)methyl)-3-methyloxazolidin-2-one

(threo-26), $\left(R^{*}\right)$-4-(( $\left.S^{*}\right)$-Hydroxy(4-methoxyphenyl)methyl)-3-methyloxazolidin-2-one

(erythro-26), 3-(2-Hydroxy-2-(4-methoxyphenyl)ethyl)oxazolidin-2-one (27): The title compounds were obtained according to the above-mentioned procedure from 4-methoxybenzaldehyde (2a) (408 mg, $3.0 \mathrm{mmol}$ ), 3-methyl-2-oxazolidinone (14) $(9 \mathrm{~mL}, 105 \mathrm{mmol})$ and $\mathrm{Et}_{3} \mathrm{~B}(2.6 \mathrm{~mL}, 18 \mathrm{mmol})$. After $36 \mathrm{~h}$, the mixture was treated with sat. $\mathrm{NH}_{4} \mathrm{Cl}$ and extracted with $\mathrm{Et}_{2} \mathrm{O}$. The separated aqueous phase was 
thoroughly extracted with $\mathrm{CH}_{2} \mathrm{Cl}_{2}$ and the combined organic extract was dried over $\mathrm{MgSO}_{4}$. After concentration in vacuo, the residue was purified by silica gel column chromatography (EtOAc/Hex 1:4) to give unreacted aldehyde $\mathbf{2 a}$ (35 mg, 9\%), ethyl adduct $\mathbf{6 a}$ (56 mg, 11\%), and 4-methoxybenzyl alcohol (7a) (37 mg, 9\%). Further elution with $\mathrm{MeOH} / \mathrm{CHCl}_{3}(1: 25)$ afforded a mixture of alcohols threo-26, erythro-26, and $27(306 \mathrm{mg}, 43 \%)$ as a colorless solid. The yields and the ratio of the products were determined by ${ }^{1} \mathrm{H}$ NMR analysis to be threo-26/erythro-26 (272 $\mathrm{mg}, 38 \%$, dr 56:44) and 27 (34 mg, 5\%). Careful purification of the mixture of alcohols threo-26, erythro-26, and $\mathbf{2 7}$ by flash silica gel chromatography ( $\left.\mathrm{MeOH} / \mathrm{CHCl}_{3} 1: 30\right)$ and subsequent recrystallization $\left(\mathrm{EtOAc} / \mathrm{CH}_{2} \mathrm{Cl}_{2}\right)$ afforded pure major threo-26 as colorless plates, minor erythro-26 as colorless plates, and regioisomeric alcohol $\mathbf{2 7}$ as a colorless solid. The relative configuration of major product threo-26 was determined by X-ray crystallographic analysis (See CIF file). threo-Alcohol 26: Mp 119-120 ${ }^{\circ} \mathrm{C}$ $\left(\right.$ EtOAc/ $\mathrm{CH}_{2} \mathrm{Cl}_{2}$ ); IR (neat) v 3404, $1730 \mathrm{~cm}^{-1} ;{ }^{1} \mathrm{H}$ NMR $\left(300 \mathrm{MHz}, \mathrm{CDCl}_{3}\right) \delta$ 7.28-7.23 (m, 2H), 6.94-6.88 (m, 2H), 4.71 (d, 1H, J=6.6 Hz), 4.01-3.88 (m, 2H), $3.83(\mathrm{~m}, 1 \mathrm{H}), 3.81(\mathrm{~s}, 3 \mathrm{H}), 3.05(\mathrm{~s}, 3 \mathrm{H})$; ${ }^{13} \mathrm{C}$ NMR $\left(100 \mathrm{MHz}, \mathrm{CDCl}_{3}\right) \delta 159.9,158.8,131.4,127.7,114.4,76.4,63.8,62.4,55.3,31.9 ; \mathrm{MS} m / z$ : $237\left(\mathrm{M}^{+}\right), 137$ (100\%); HRMS (EI) calcd for $\mathrm{C}_{12} \mathrm{H}_{15} \mathrm{O}_{4} \mathrm{~N}\left(\mathrm{M}^{+}\right)$: 237.1001, found: 237.0999 . erythro-Alcohol 26: Mp 138-139 ${ }^{\circ} \mathrm{C}\left(\mathrm{EtOAc} / \mathrm{CH}_{2} \mathrm{Cl}_{2}\right)$; IR (neat) $v 3393,1734 \mathrm{~cm}^{-1}$; ${ }^{1} \mathrm{H}$ NMR (300 $\left.\mathrm{MHz}, \mathrm{CDCl}_{3}\right) \delta$ 7.30-7.26 (m, 2H), 6.94-6.89 (m, 2H), $4.90(\mathrm{~d}, 1 \mathrm{H}, \mathrm{J}=4.0 \mathrm{~Hz}), 4.35$ (dd, 1H, J=8.9, 5.8 $\mathrm{Hz}), 4.10(\mathrm{t}, 1 \mathrm{H}, J=8.9 \mathrm{~Hz}), 3.84(\mathrm{~m}, 1 \mathrm{H}), 3.81(\mathrm{~s}, 3 \mathrm{H}), 2.80(\mathrm{~s}, 3 \mathrm{H}) ;{ }^{13} \mathrm{C} \mathrm{NMR}\left(75 \mathrm{MHz}, \mathrm{CDCl}_{3}\right) \delta$ 159.6, 159.2, 131.1, 127.2, 114.3, 70.9, 63.1, 62.9, 55.3, 30.0; MS m/z: $237\left(\mathrm{M}^{+}\right), 137$ (100\%); HRMS (EI) calcd for $\mathrm{C}_{12} \mathrm{H}_{15} \mathrm{O}_{4} \mathrm{~N}\left(\mathrm{M}^{+}\right)$: 237.1001, found: 237.1003. Alcohol 27: Mp 113-114 ${ }^{\circ} \mathrm{C}$; IR (neat) $v$ 3404, $1728 \mathrm{~cm}^{-1}$; ${ }^{1} \mathrm{H}$ NMR $\left(400 \mathrm{MHz}, \mathrm{CDCl}_{3}\right) \delta$ 7.34-7.30 (m, 2H), 6.92-6.88 (m, 2H), $4.93(\mathrm{dd}, 1 \mathrm{H}$, $J=7.7,4.2 \mathrm{~Hz}), 4.30$ (t, 2H, $J=8.2 \mathrm{~Hz}), 3.81$ (s, 3H), 3.63 (dt, 1H, $J=8.5,7.7 \mathrm{~Hz}), 3.55-3.41(\mathrm{~m}, 3 \mathrm{H})$; ${ }^{13} \mathrm{C} \mathrm{NMR}\left(100 \mathrm{MHz}, \mathrm{CDCl}_{3}\right) \delta 159.4,159.2,133.5,127.0,114.0,72.6,62.2,55.3,52.1,46.4 ; \mathrm{MS} m / z$ : $237\left(\mathrm{M}^{+}\right), 137(100 \%)$; HRMS (EI) calcd for $\mathrm{C}_{12} \mathrm{H}_{15} \mathrm{O}_{4} \mathrm{~N}\left(\mathrm{M}^{+}\right)$: 237.1001, found: 237.0997.

$N$-(2-Hydroxy-2-(4-methoxyphenyl)ethyl)- $N$-methylacetamide (28): The title compound was obtained according to the above-mentioned procedure from 4-methoxybenzaldehyde (2a) (408 mg, 3.0 mmol), $N, N$-dimethylacetamide (15) $(20 \mathrm{~mL}, 210 \mathrm{mmol})$ and $\mathrm{Et}_{3} \mathrm{~B}(2.6 \mathrm{~mL}, 18 \mathrm{mmol})$. After $41 \mathrm{~h}$, sat. 
$\mathrm{NH}_{4} \mathrm{Cl}$ was added and the mixture was extracted with $\mathrm{CH}_{2} \mathrm{Cl}_{2}$. The organic extract was dried over $\mathrm{MgSO}_{4}$ and concentrated in vacuo. The purification by silica gel column chromatography (EtOAc/Hex 1:4) gave unreacted aldehyde $\mathbf{2 a}(76 \mathrm{mg}, 19 \%)$ as a colorless oil, ethyl adduct $\mathbf{6 a}(68 \mathrm{mg}, 14 \%)$ as a colorless oil, 4-methoxybenzyl alcohol (7a) (30 mg, 7\%) as a colorless oil. Further elution with $\mathrm{MeOH} / \mathrm{EtOAc}(1: 40)$ gave alcohol 28 (265 mg, 40\%) as a colorless solid. Alcohol 28: Mp 93-94 ${ }^{\circ} \mathrm{C}$; IR (neat) v 3383, $1616 \mathrm{~cm}^{-1} ;{ }^{1} \mathrm{H}$ NMR $\left(400 \mathrm{MHz}, \mathrm{CDCl}_{3}, *\right.$ minor rotational isomer) $\delta$ 7.30-7.24 (m, 2H), 6.90-6.86 (m, 2H), 4.91 (dt, 0.75H, J=8.0, 3.5 Hz), 4.83* (dt, 0.25H, J=8.0, 3.5 Hz), 4.33 (d, 1H, $J=3.4 \mathrm{~Hz}), 3.79(\mathrm{~s}, 3 \mathrm{H}), 3.65(\mathrm{dd}, 0.75 \mathrm{H}, J=14.0,8.0 \mathrm{~Hz}), 3.58^{*}(\mathrm{dd}, 0.25 \mathrm{H}, J=14.0,8.0 \mathrm{~Hz}), 3.50(\mathrm{dd}$, 0.75H, $J=14.0,3.5 \mathrm{~Hz}), 3.31 *(\mathrm{dd}, 0.25 \mathrm{H}, J=14.0,3.5 \mathrm{~Hz}), 2.95^{*}(\mathrm{~s}, 0.75 \mathrm{H}), 2.88$ (s, 2.25H), 2.09 (s, 2.25H), 1.99* (s, 0.75H); ${ }^{13} \mathrm{C}$ NMR $\left(100 \mathrm{MHz}, \mathrm{CDCl}_{3}\right) \delta 173.1,171.4,159.4,159.0,134.4,133.7$, $126.9,126.8,114.0,113.7,73.2,71.6,58.4,57.4,55.30,55.27,38.4,34.3,21.8,21.5 ;$ MS m/z: 223 $\left(\mathrm{M}^{+}\right), 137$ (100\%); HRMS (EI) calcd for $\mathrm{C}_{12} \mathrm{H}_{17} \mathrm{O}_{3} \mathrm{~N}\left(\mathrm{M}^{+}\right)$: 223.1208, found: 223.1211 .

\section{References}

1. Soai, K.; Ookawa, A.; Kaba, T.; Ogawa, K. J. Am. Chem. Soc. 1987, 109, 7111-7115.

2. (a) Yang, X.-W.; Sheng, J.-H.; Da, C.-S.; Wang, H.-S.; Su, W.; Wang, R.; Chan, A. S. C. J. Org. Chem. 2000, 65, 295-296. (b) Alesso, E.; Torviso, R.; Lantano, B.; Erlich, M.; Finkielsztein, L. M.; Moltrasio, G.; Aguirre, J. M.; Brunet, E. ARKIVOC 2003, 283-297. 


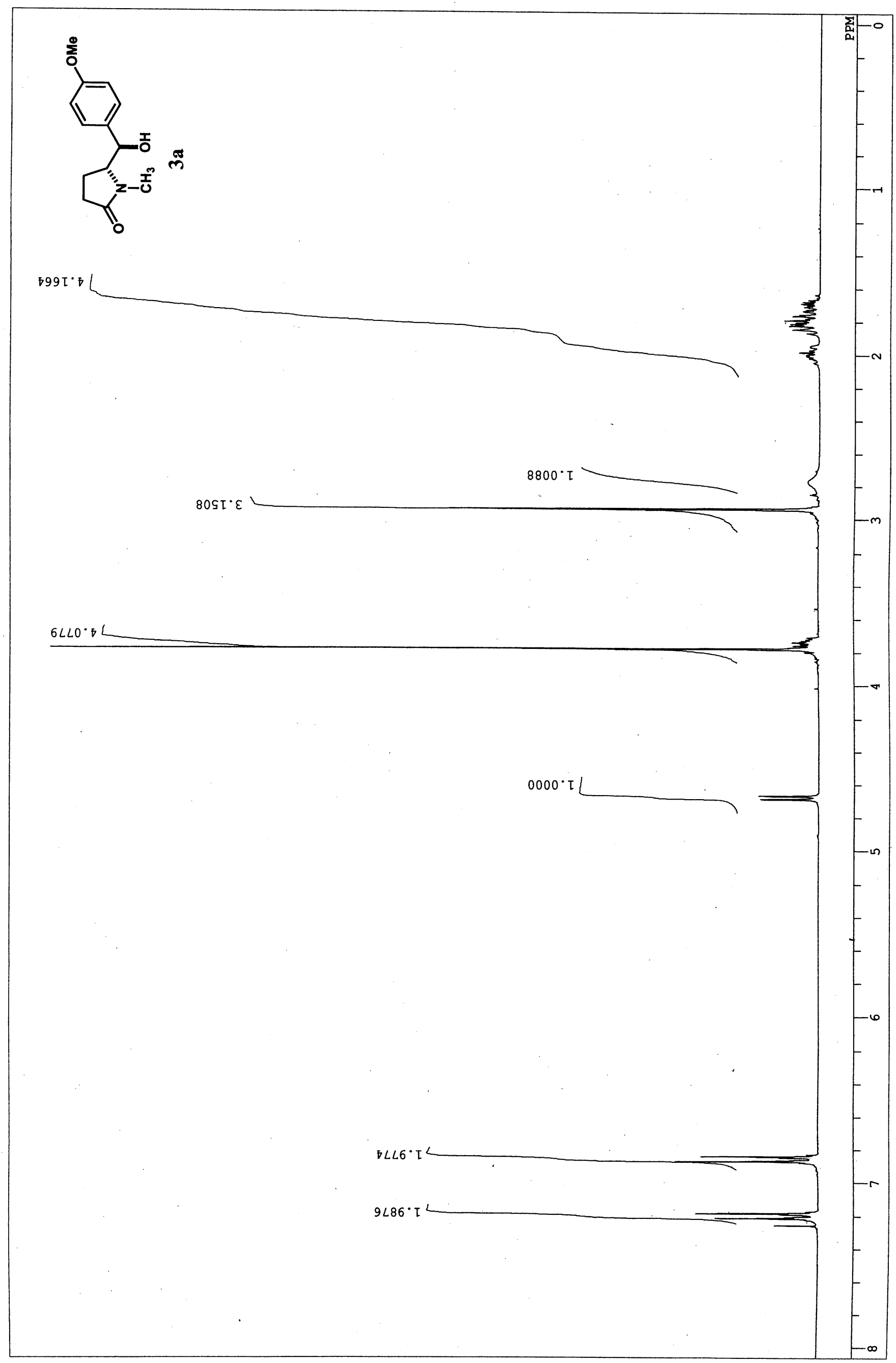




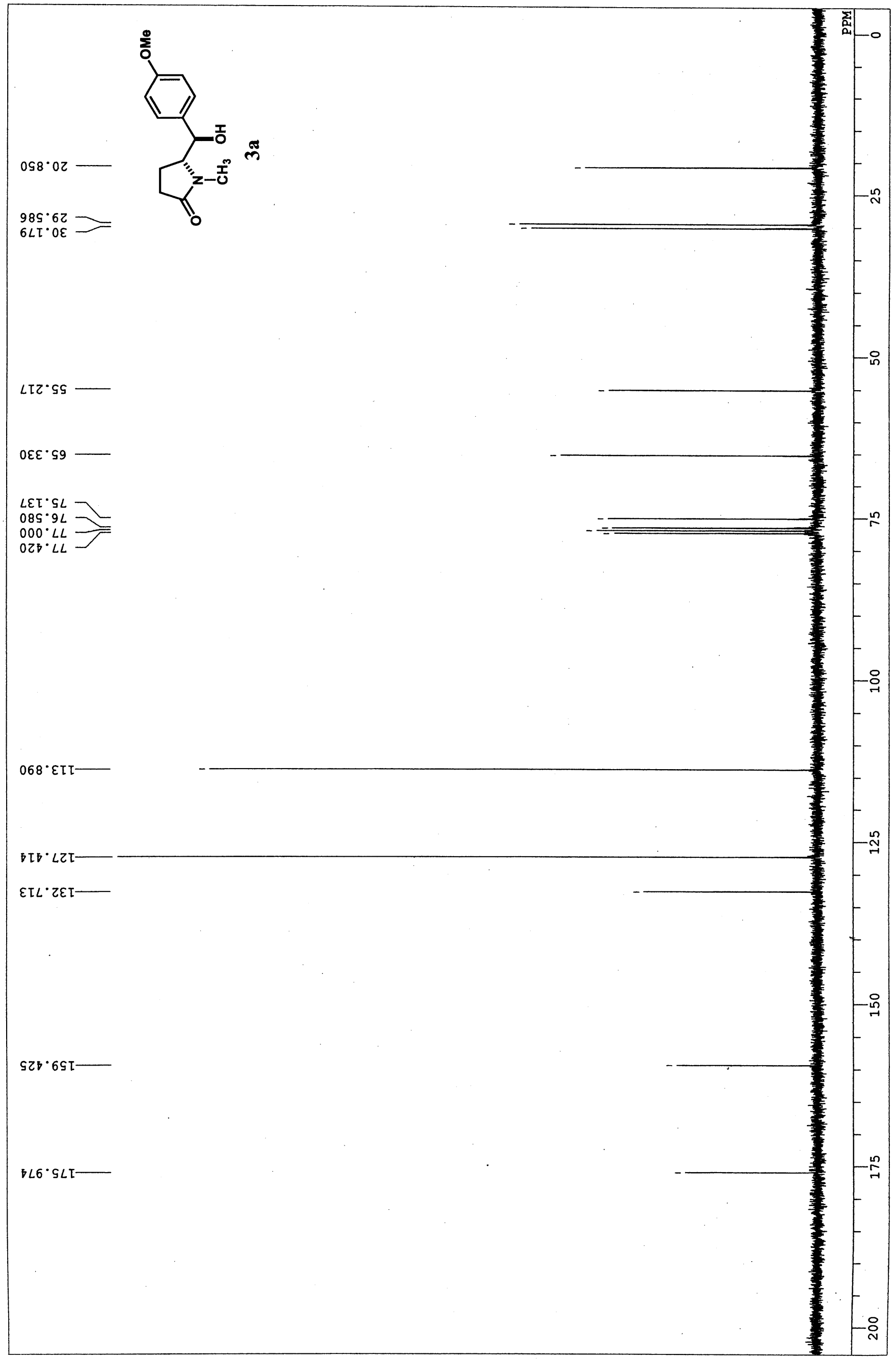




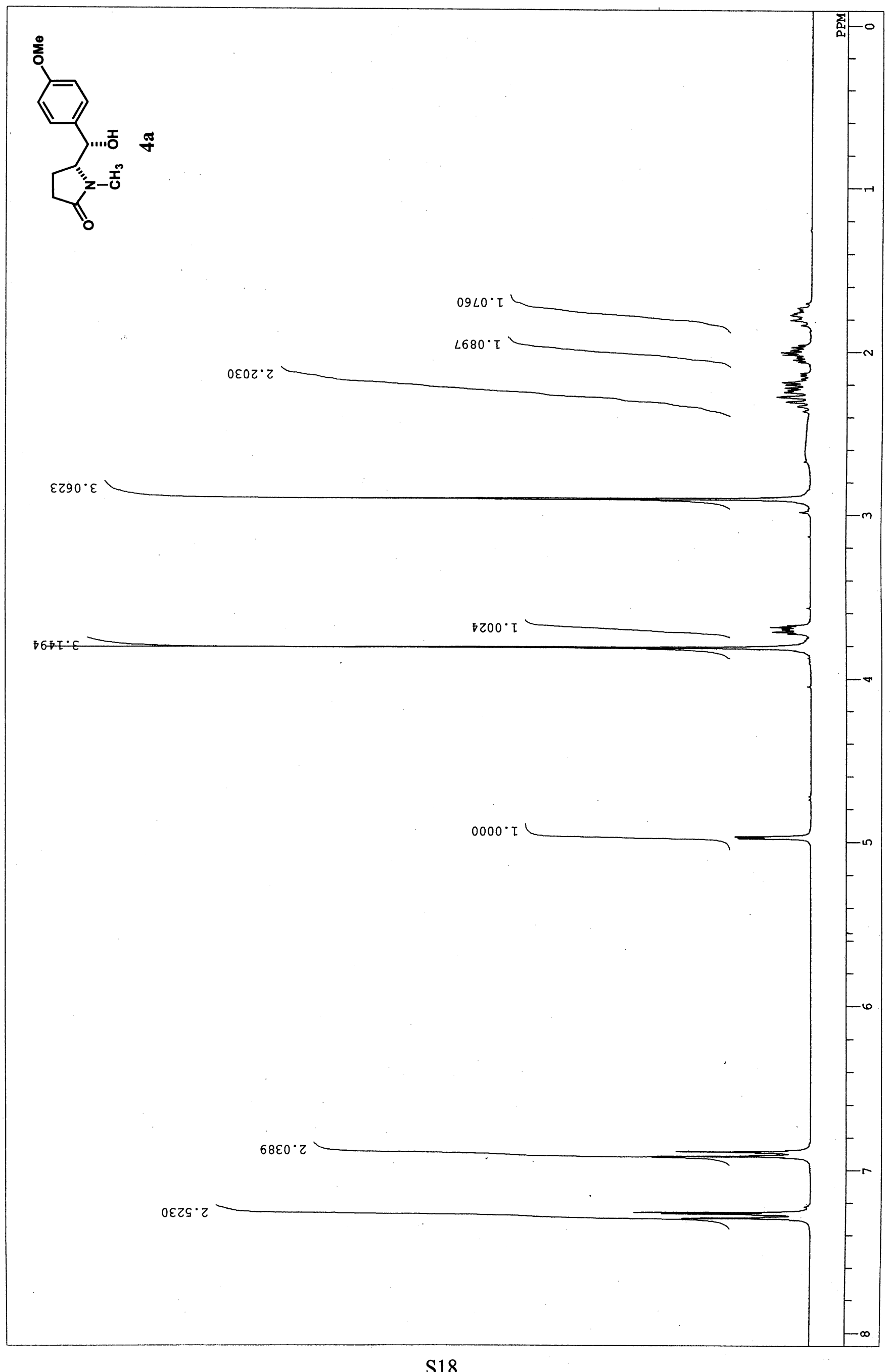




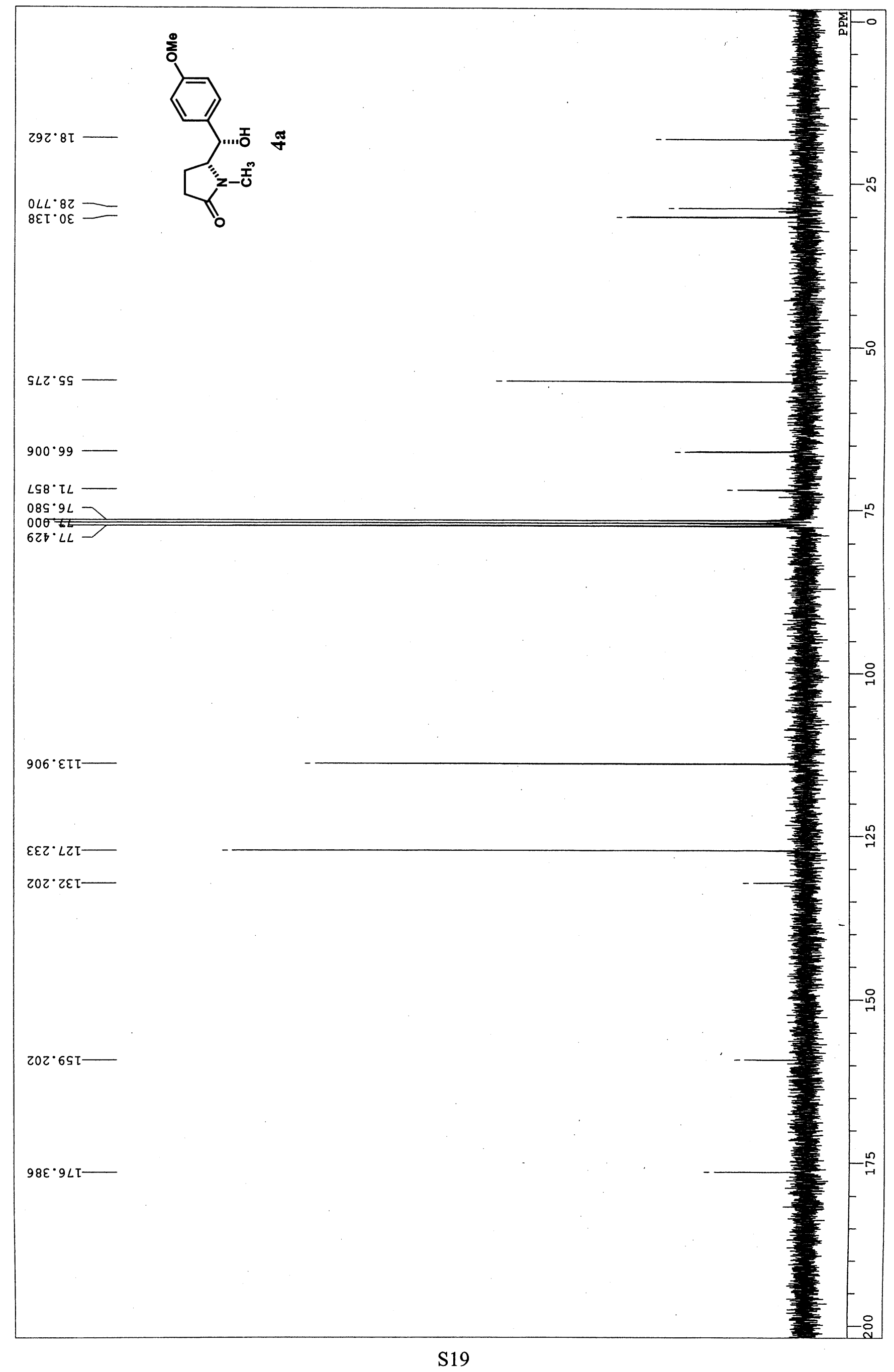




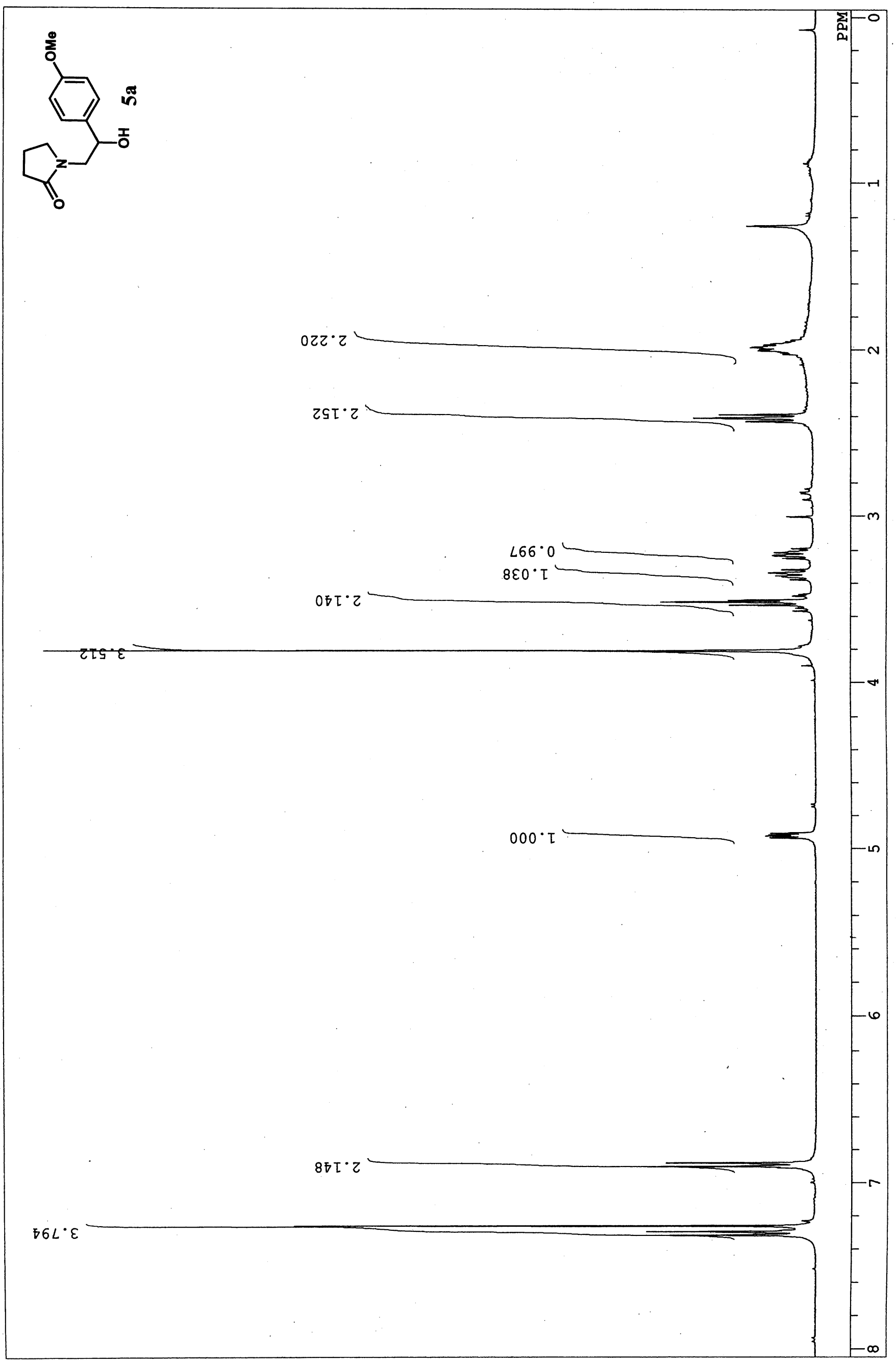




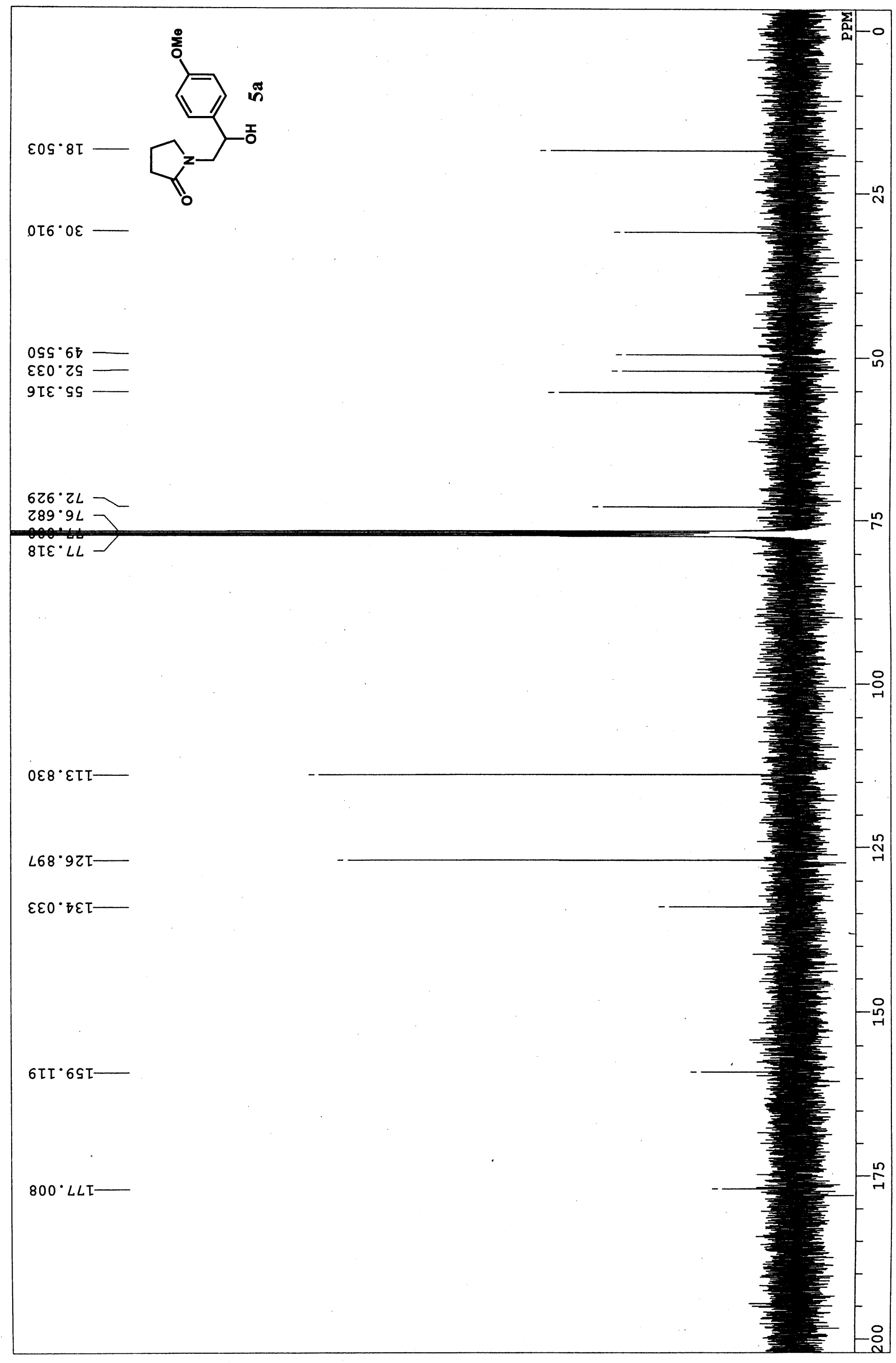




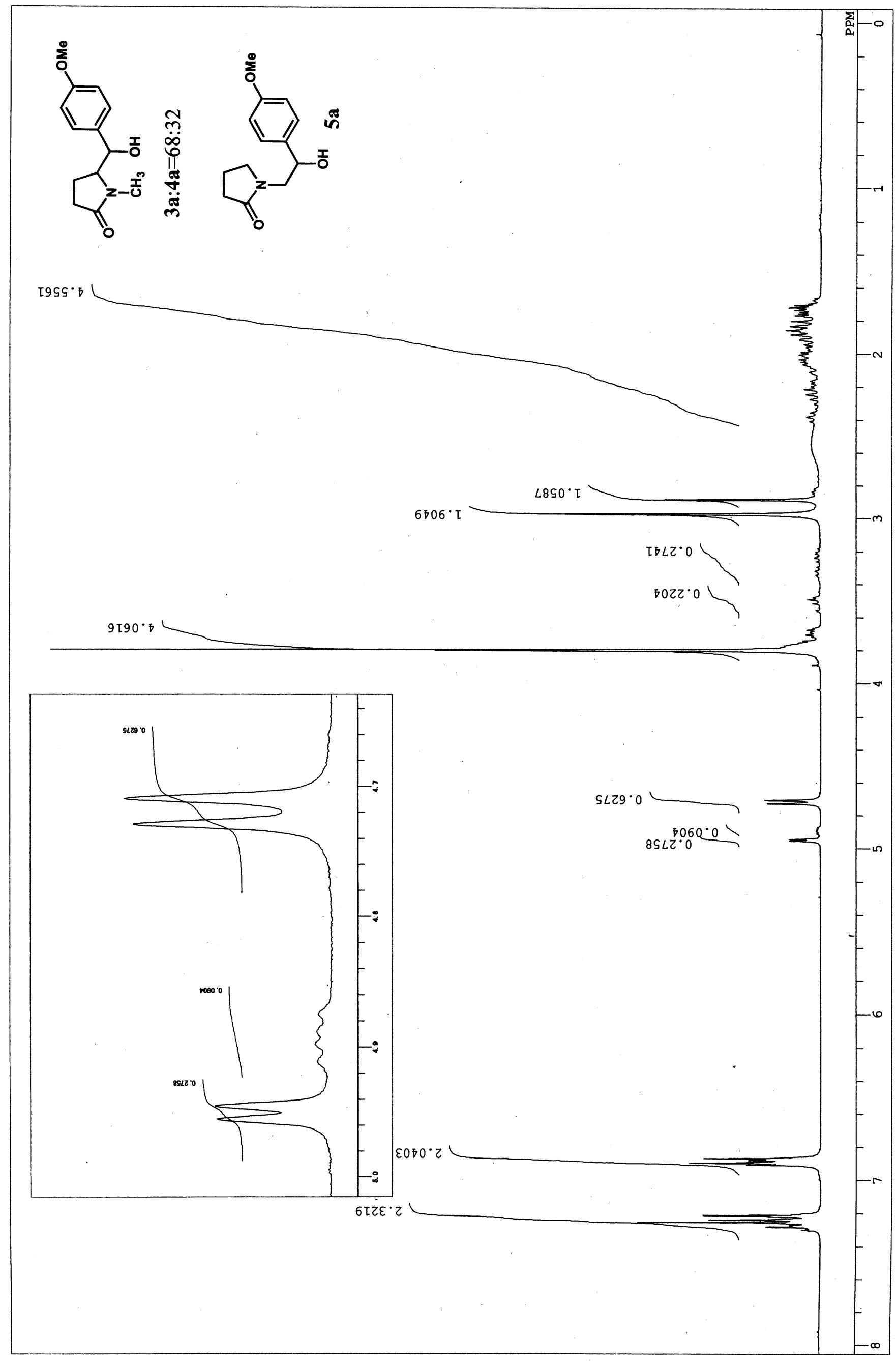




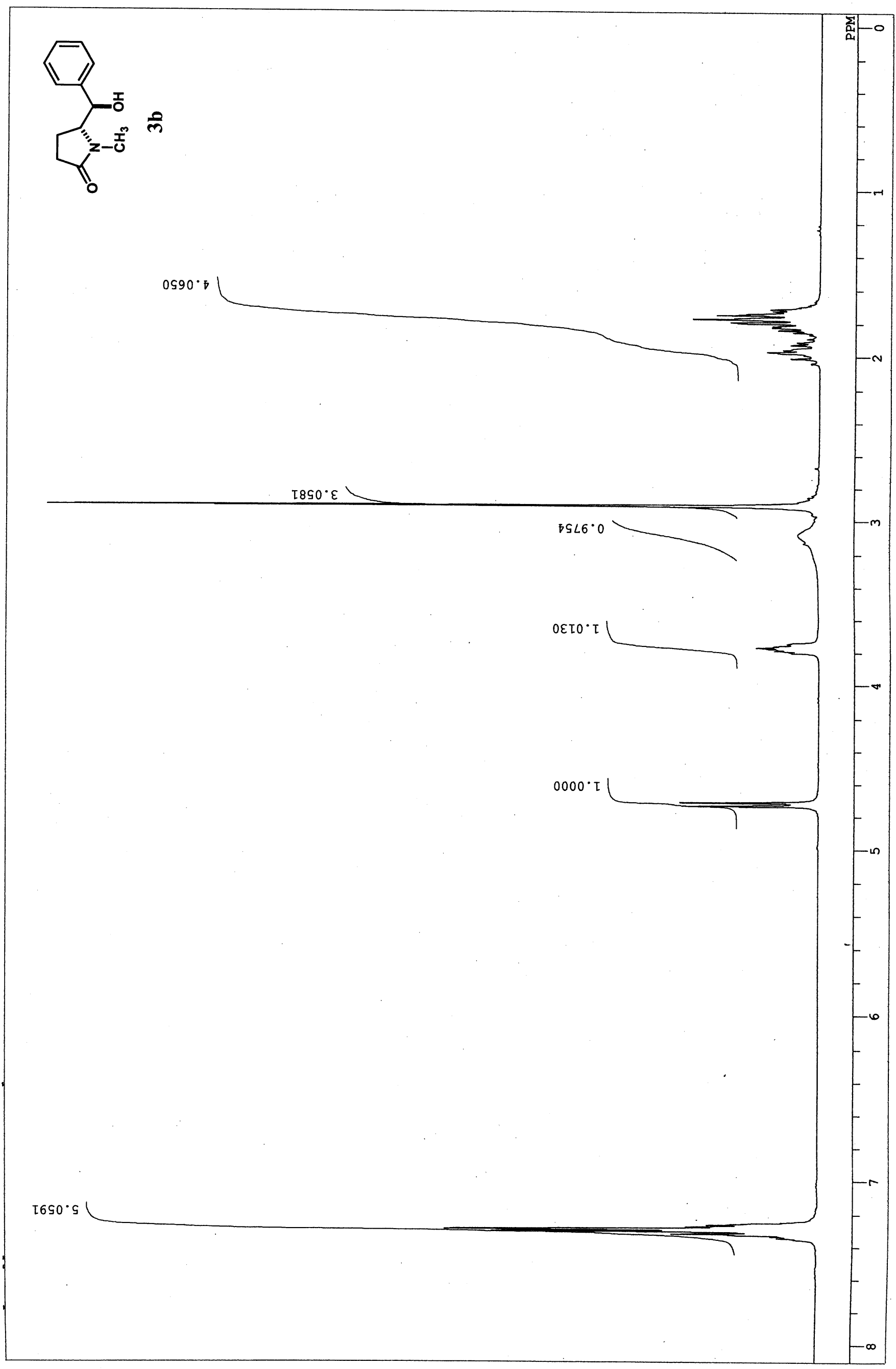




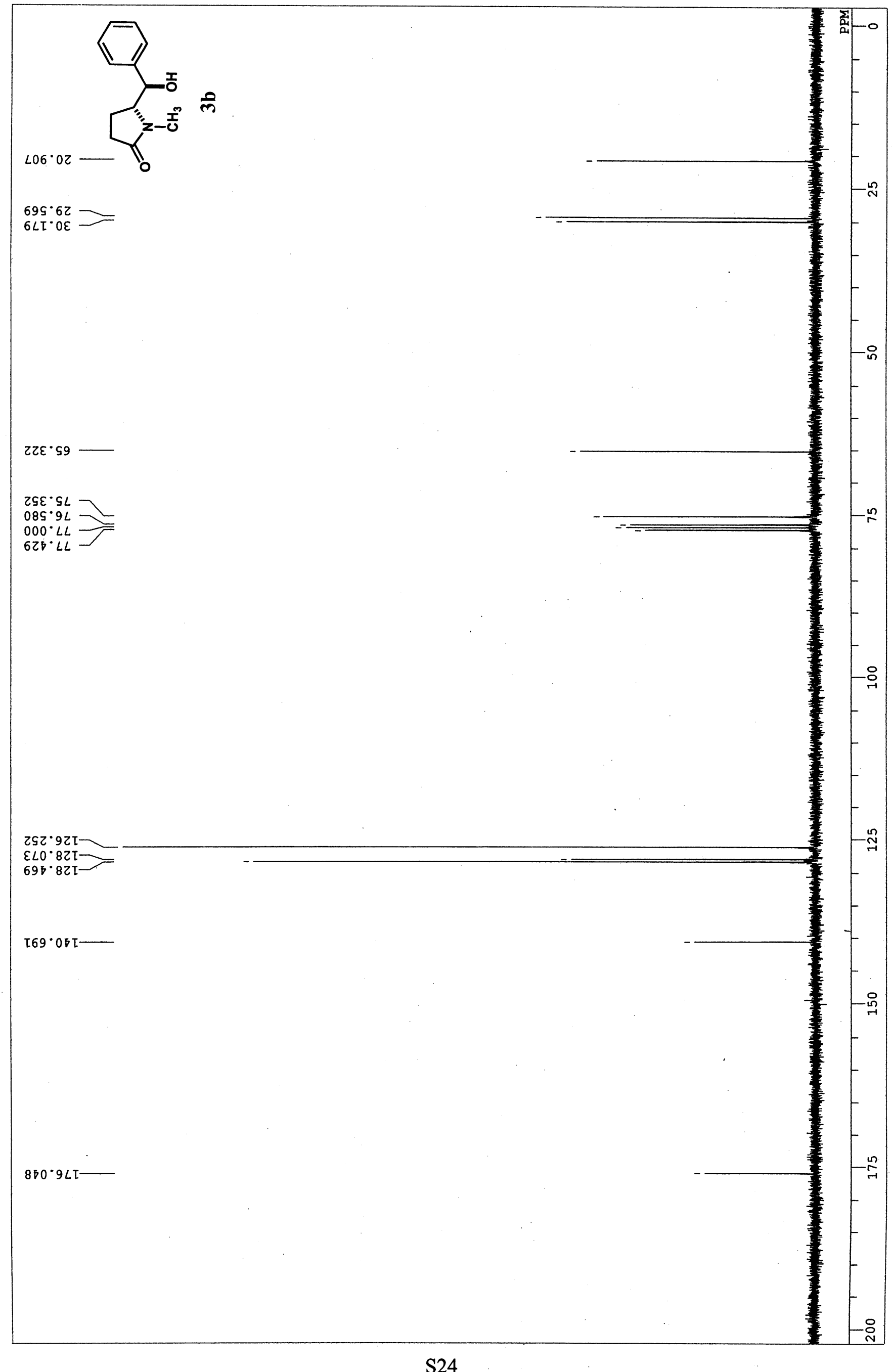




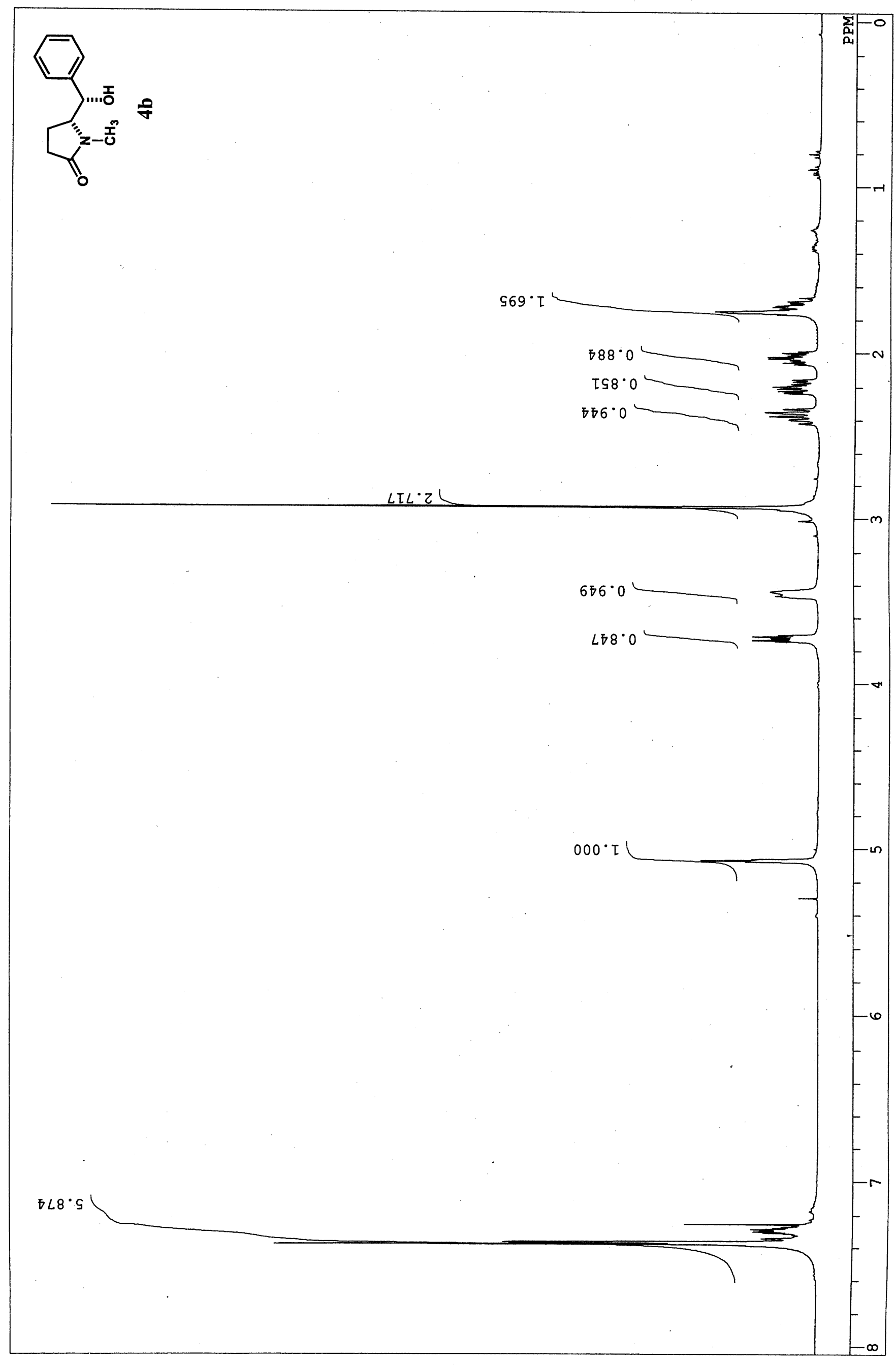




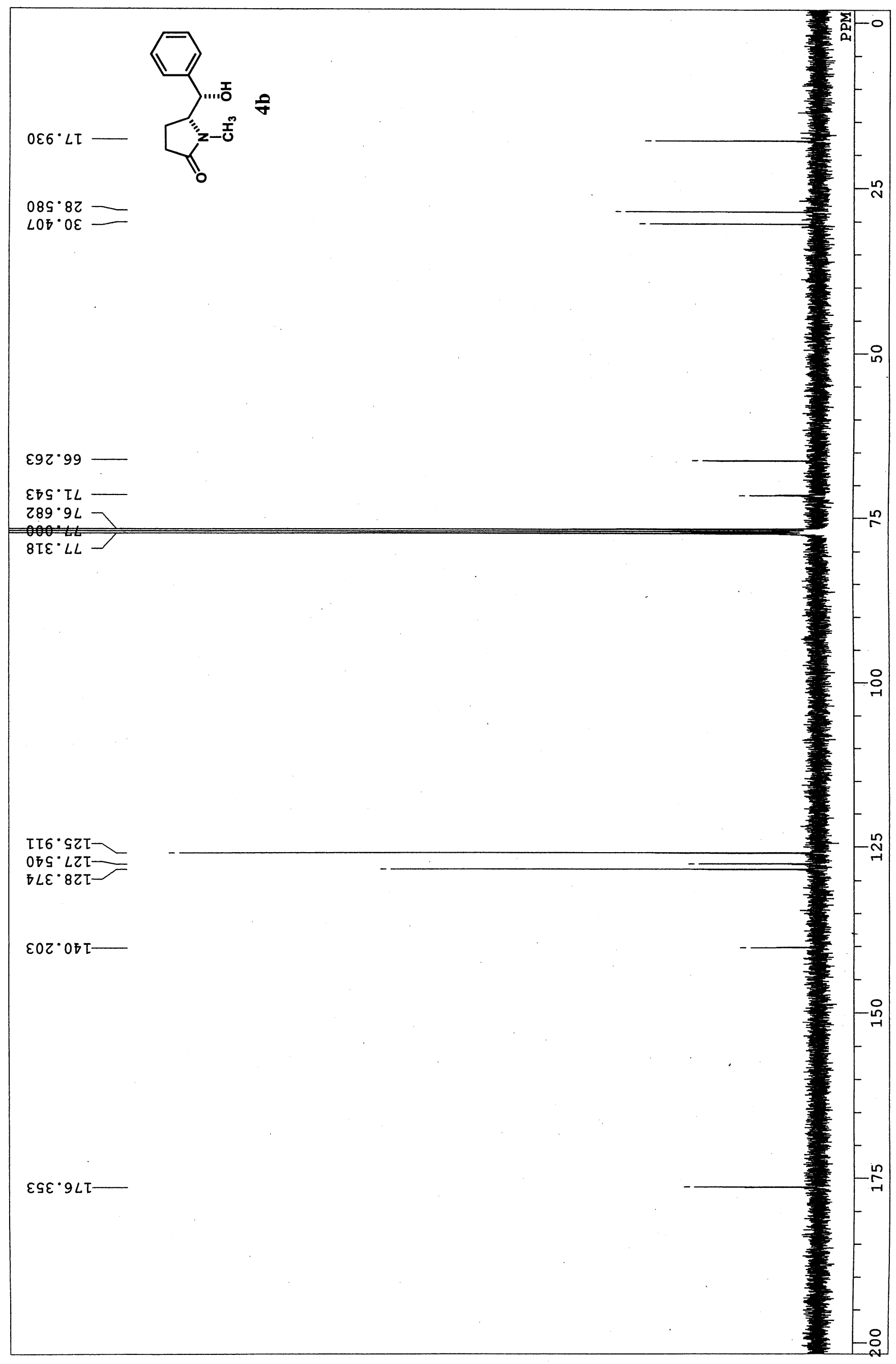




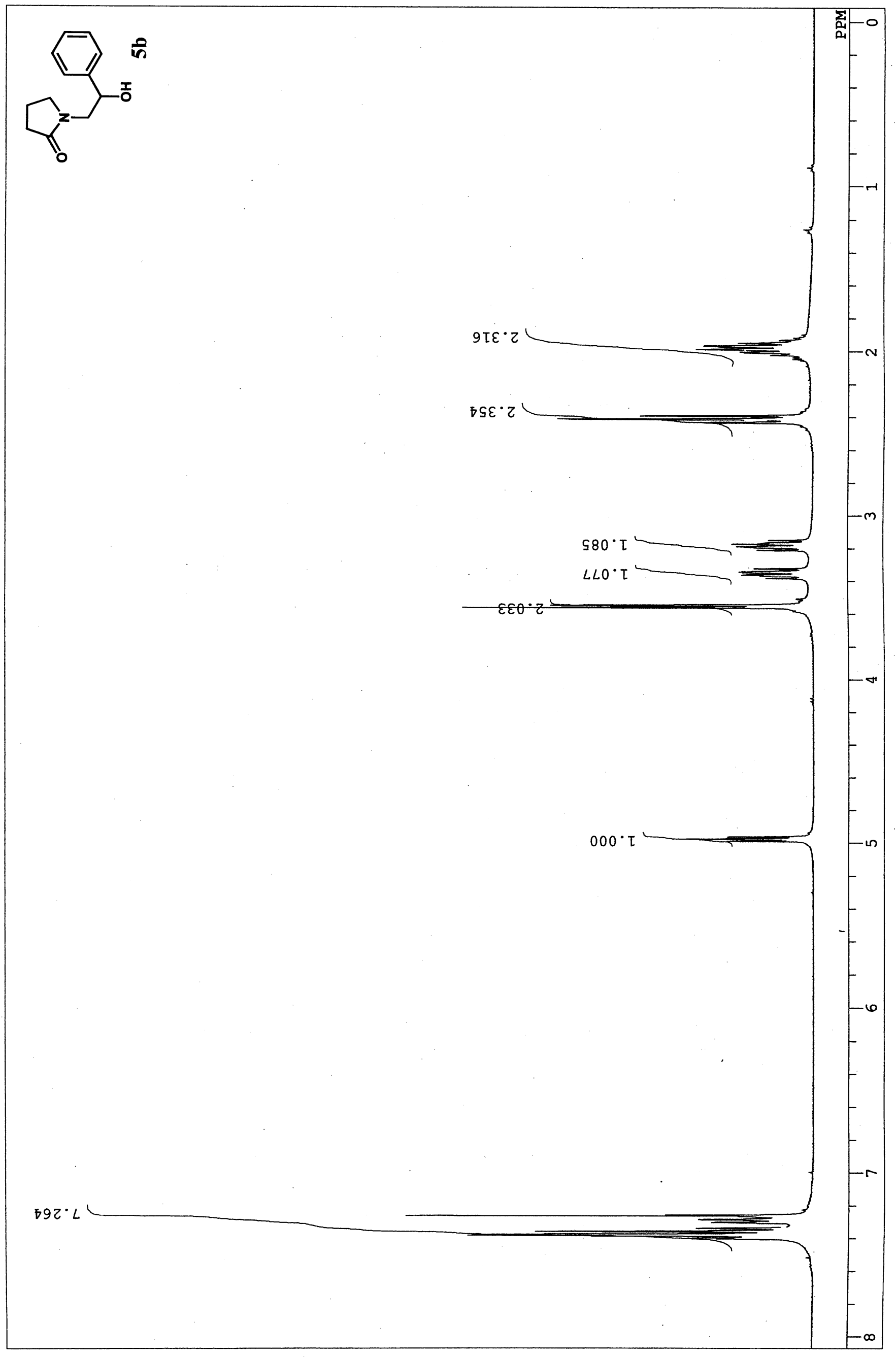




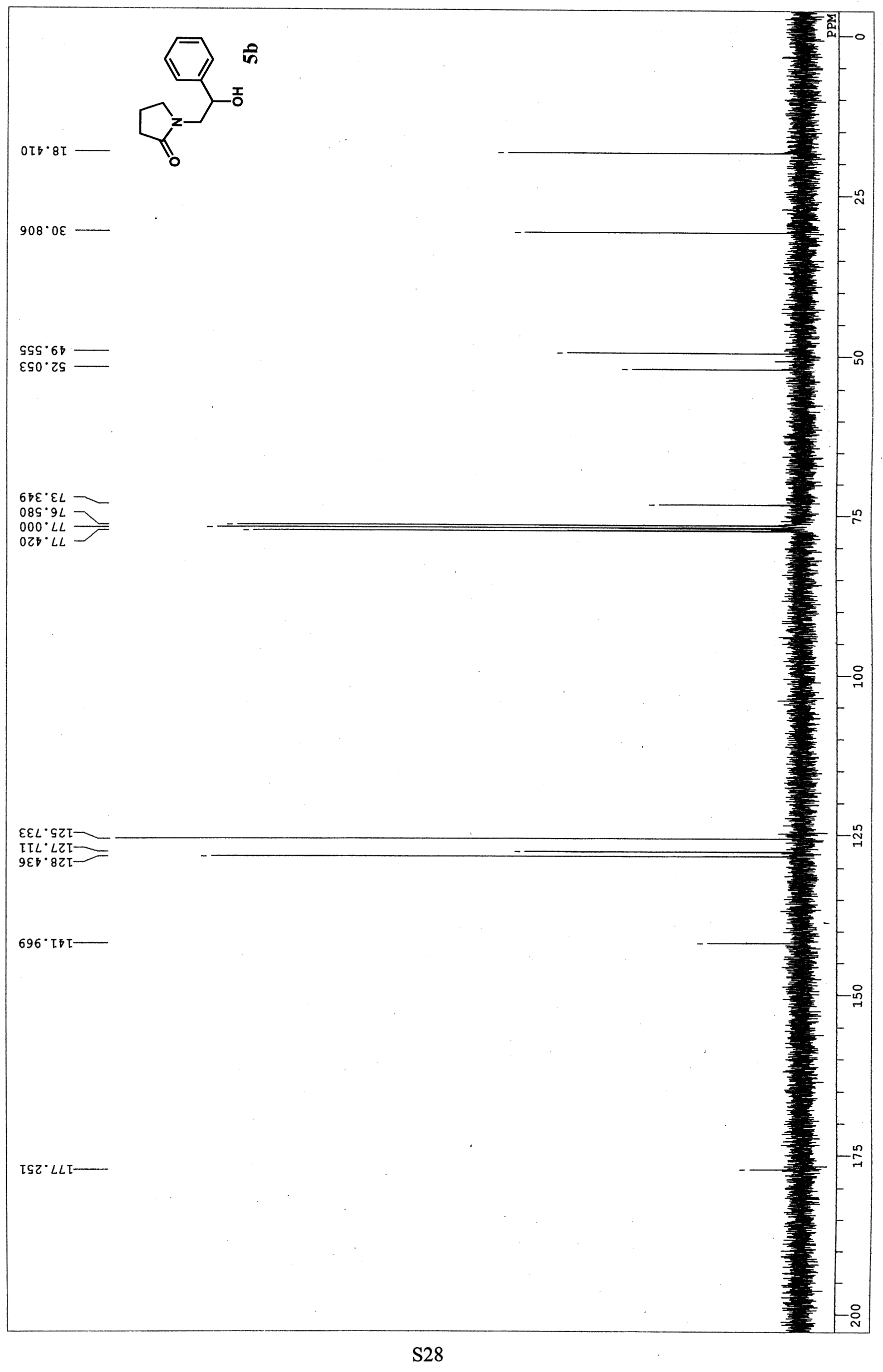




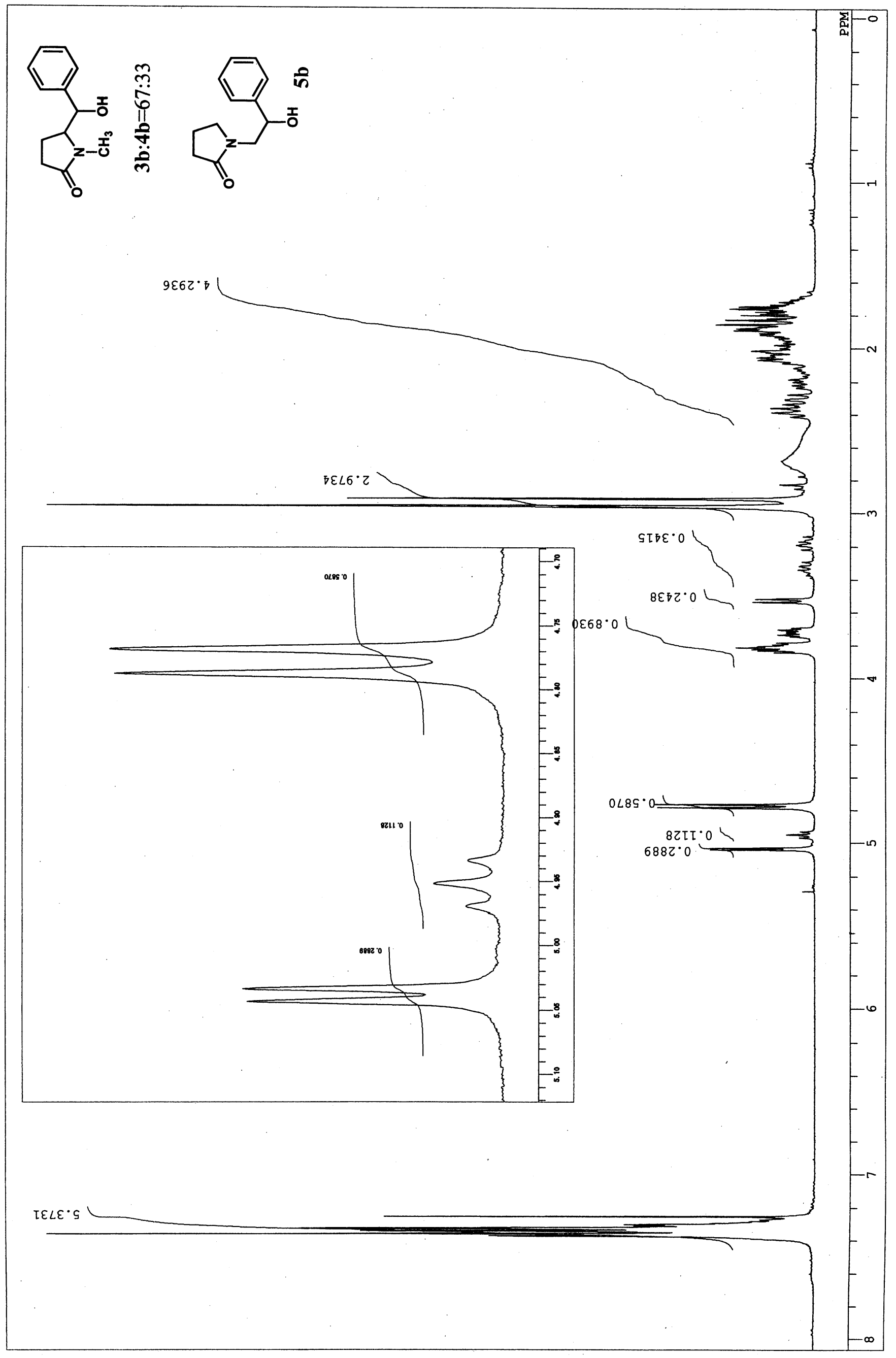




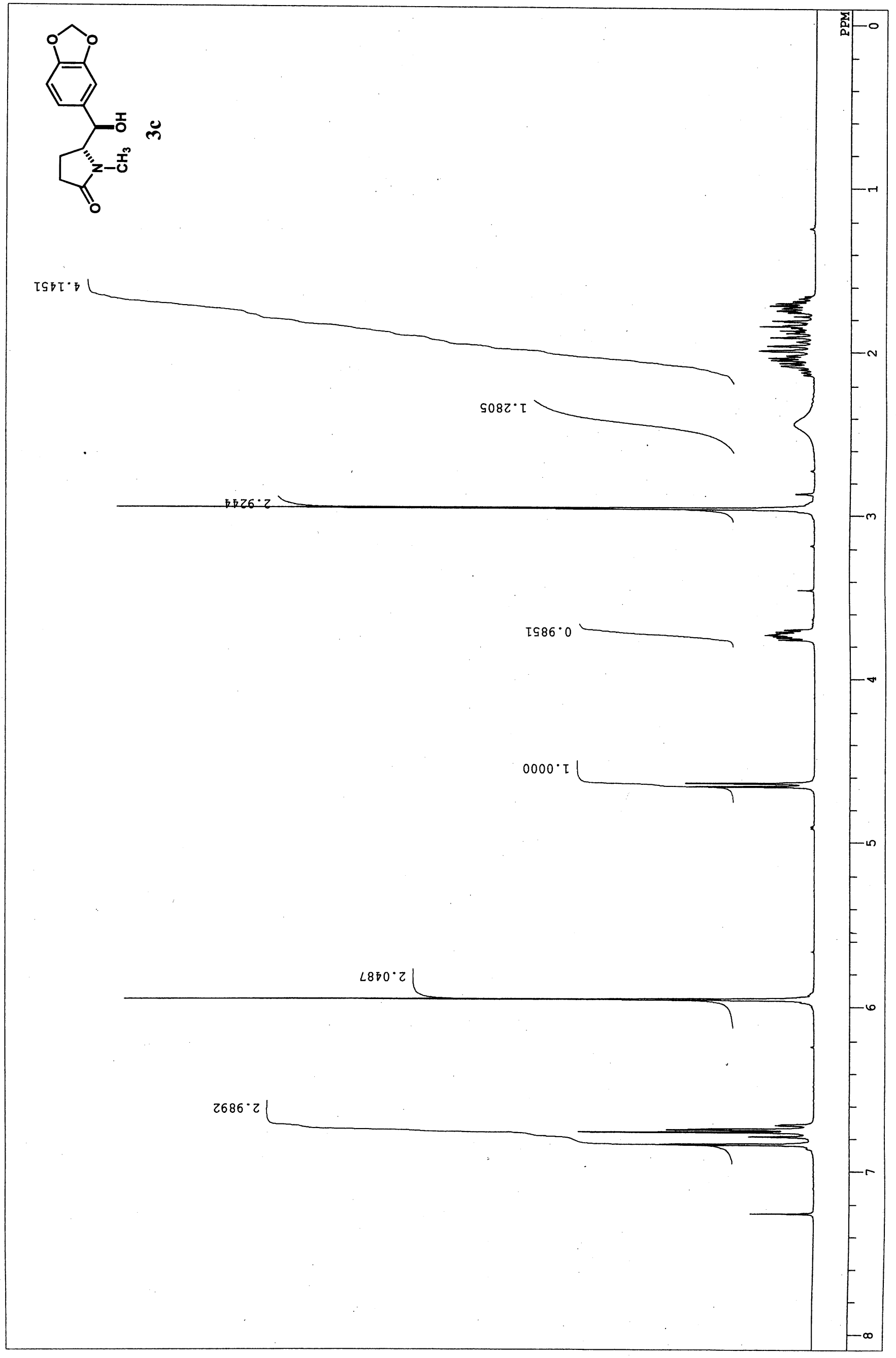




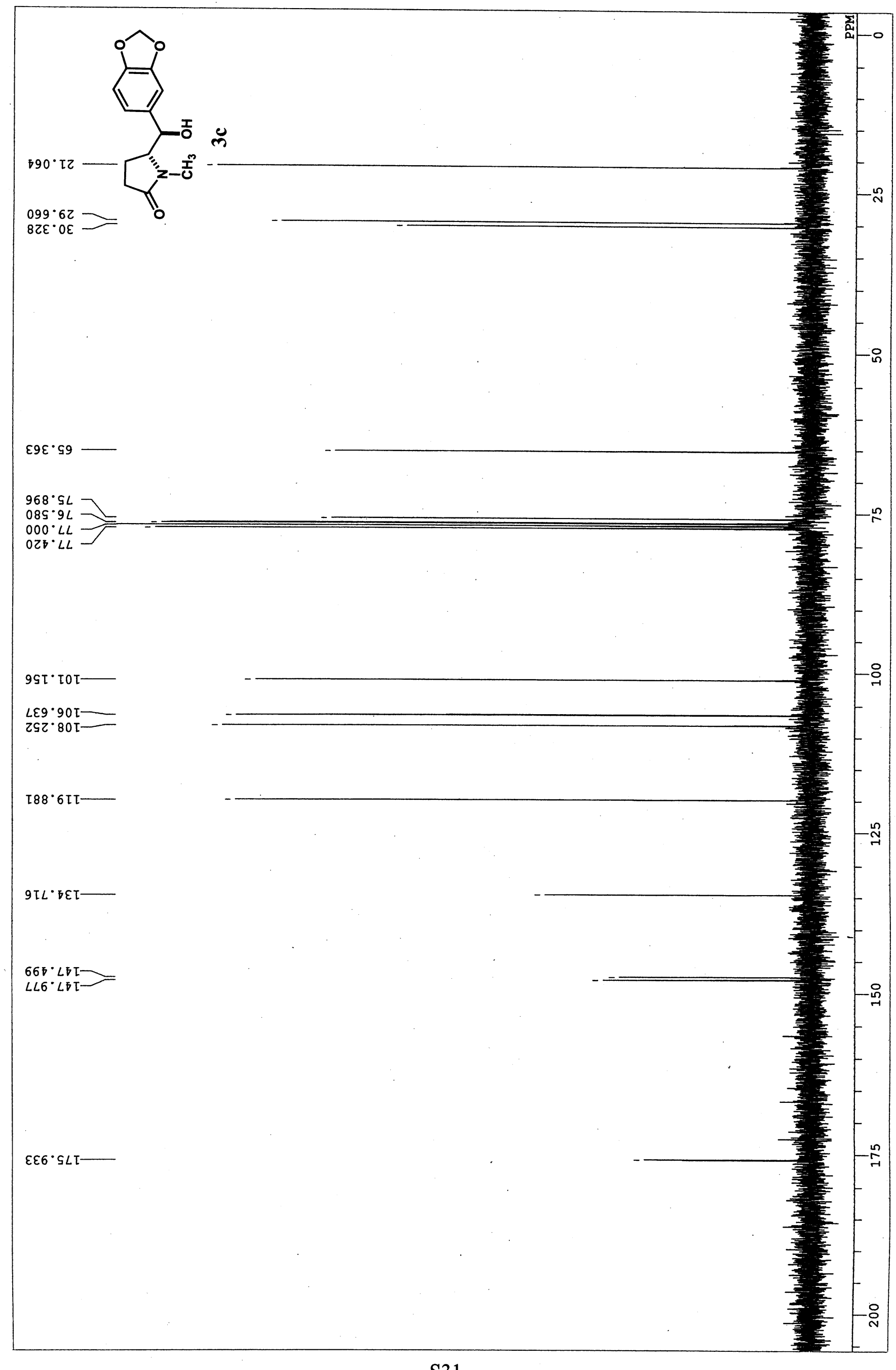




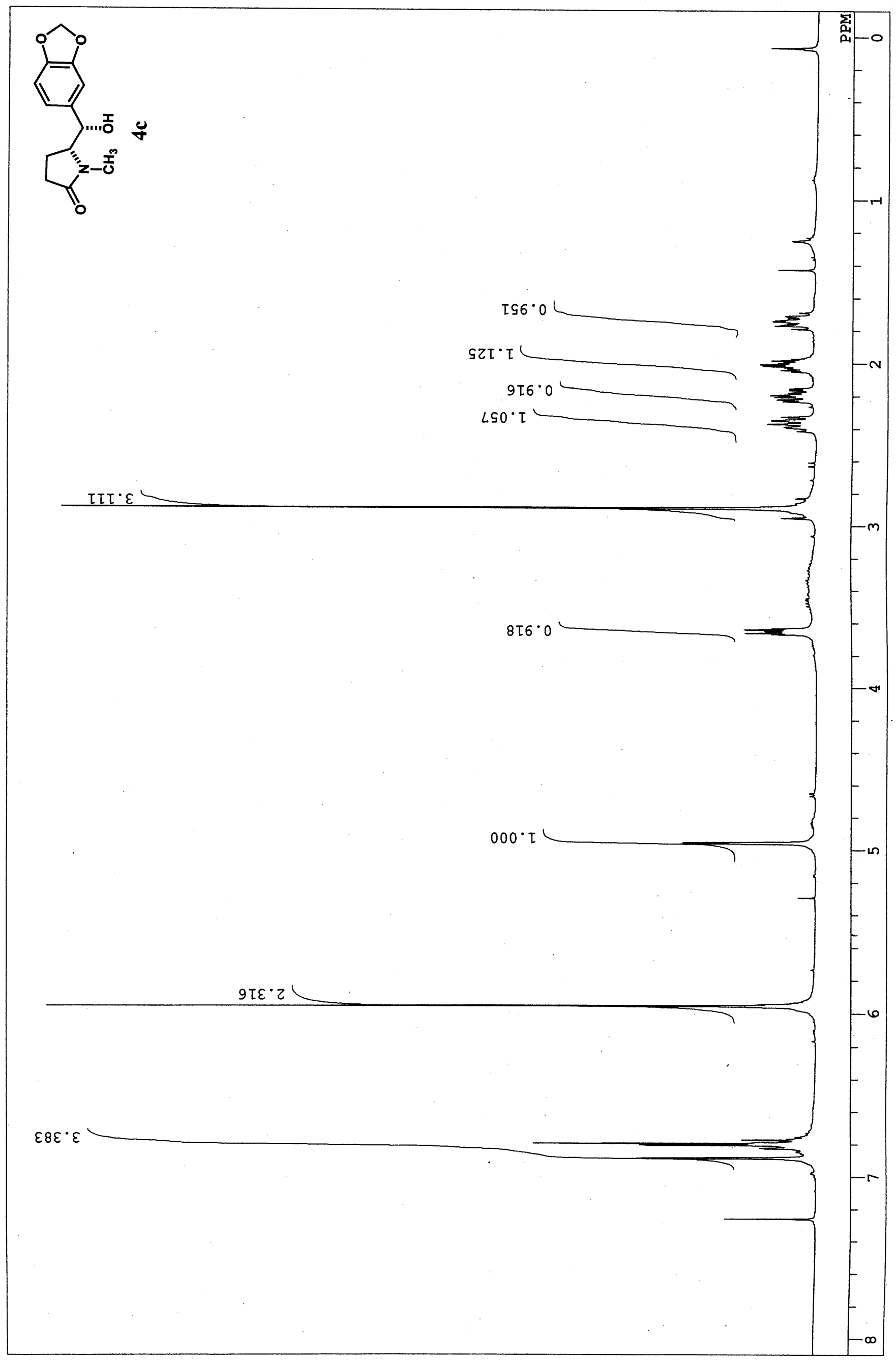




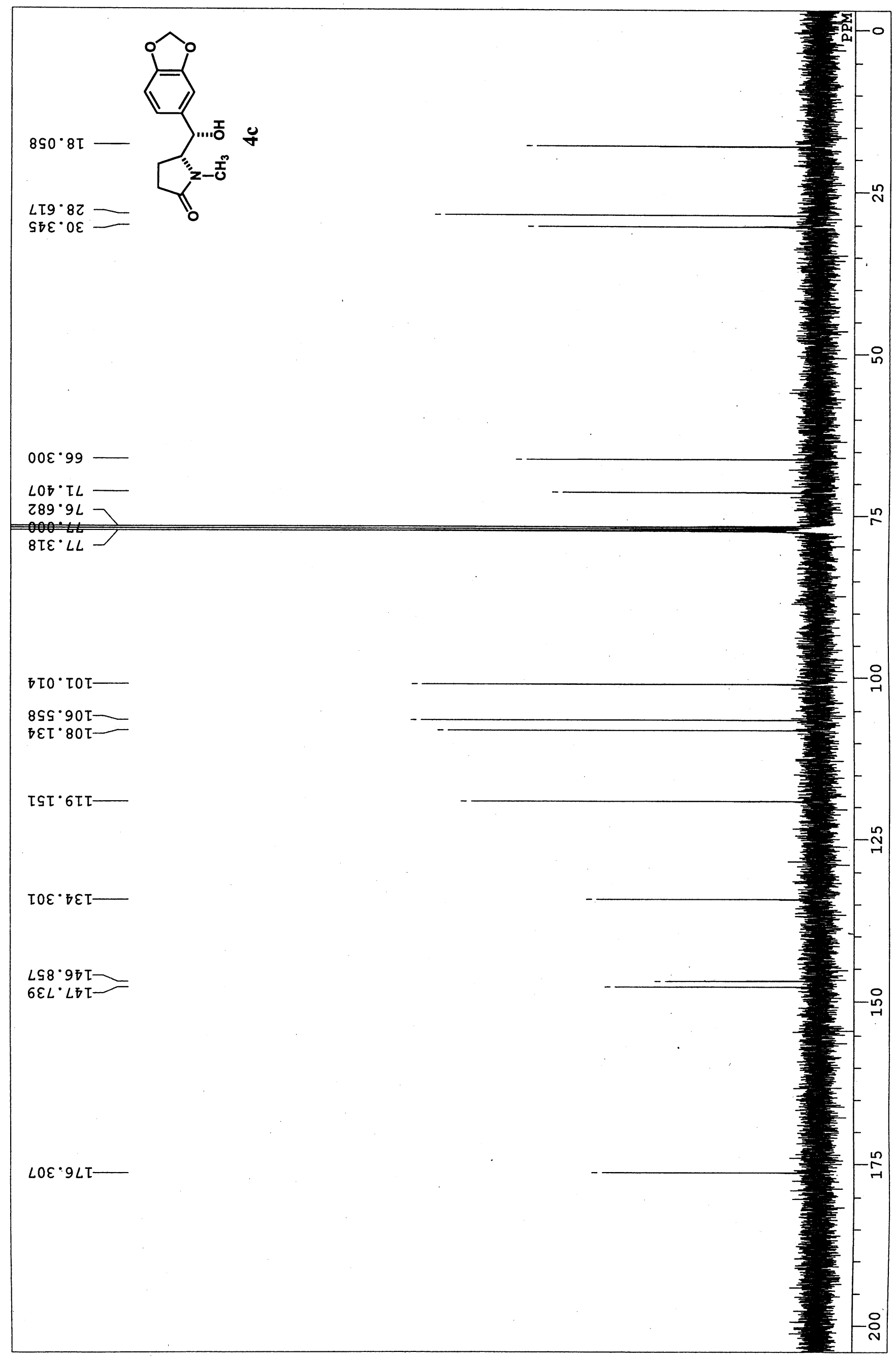




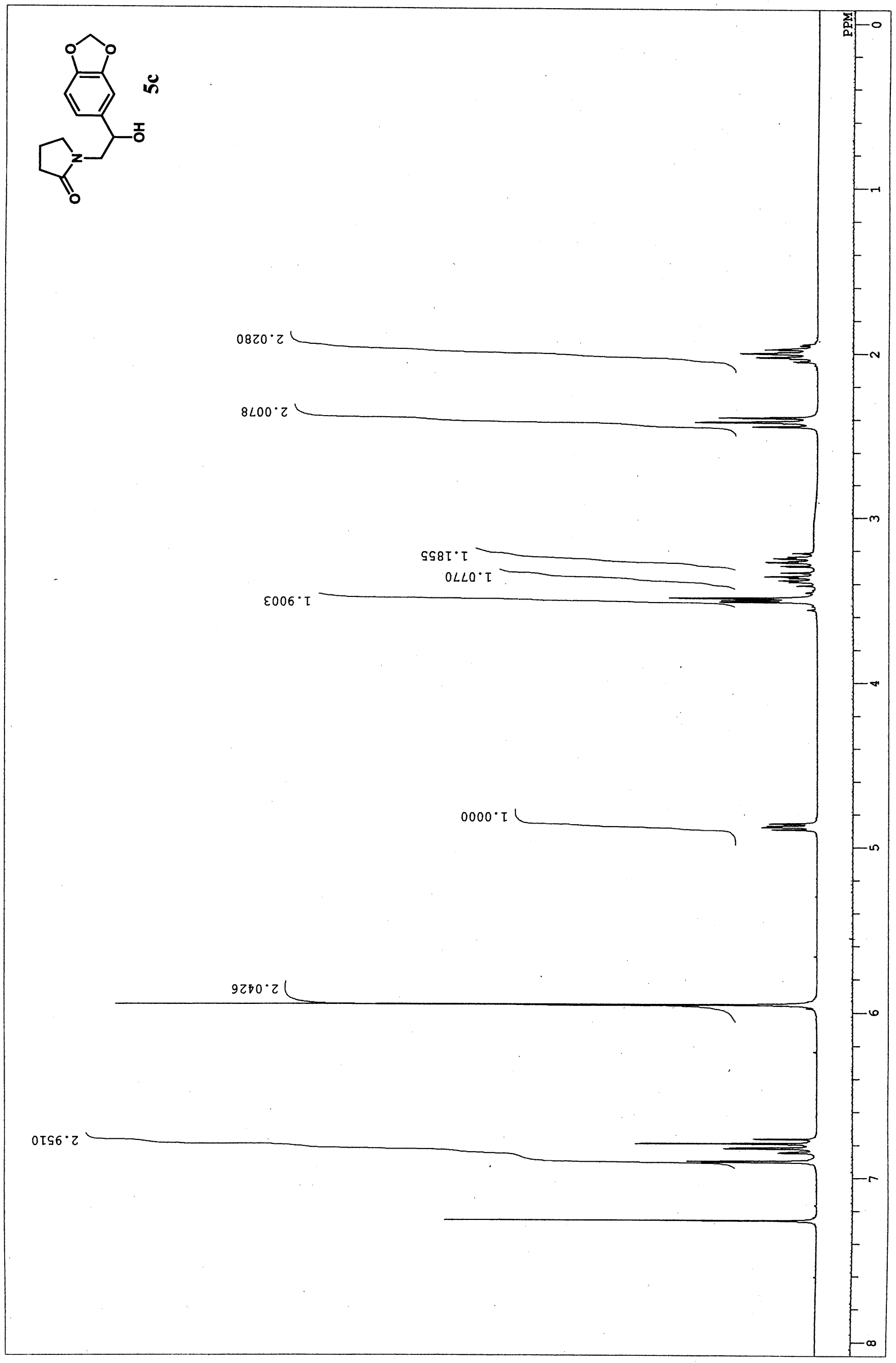




$$
1
$$




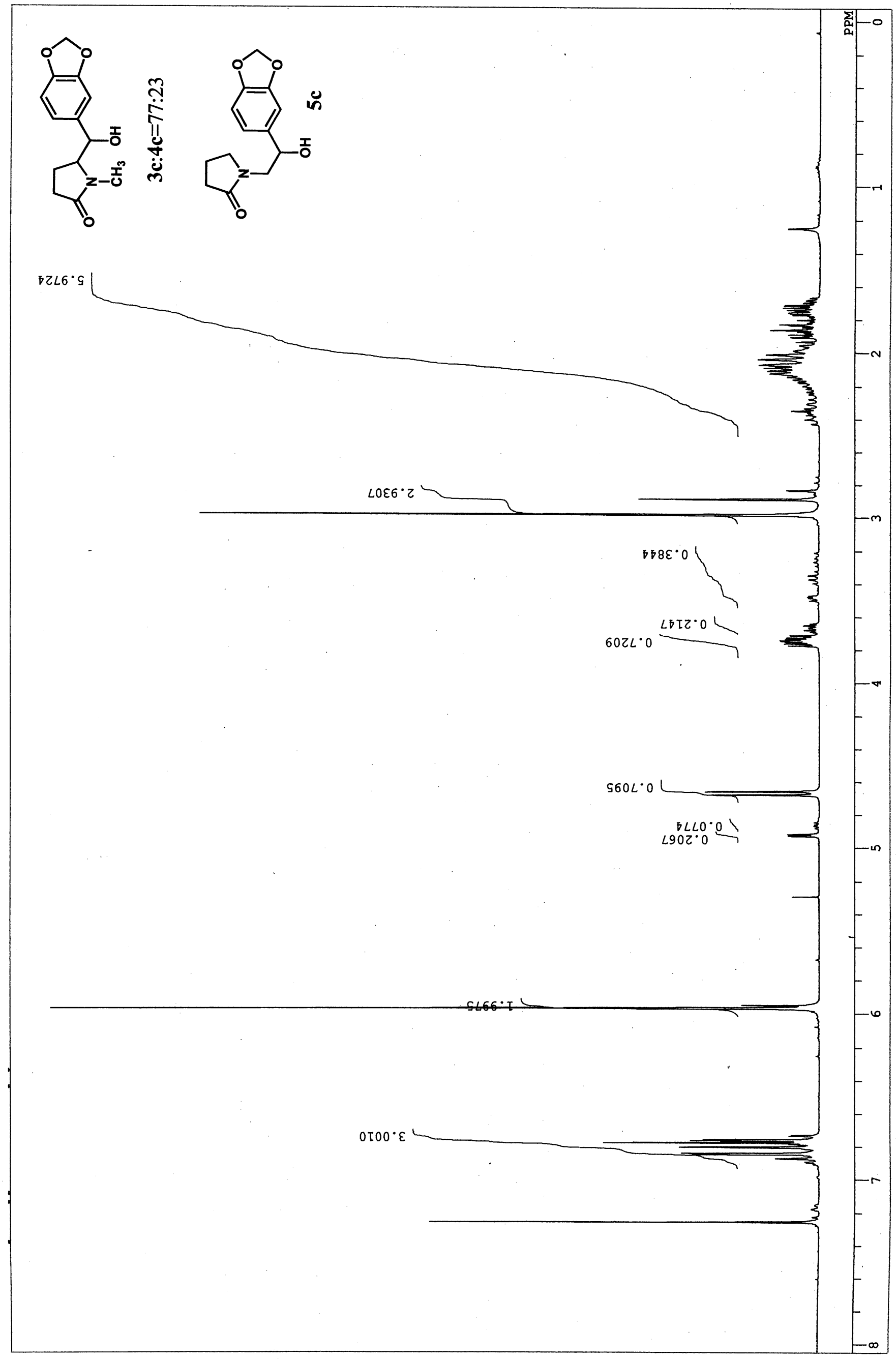




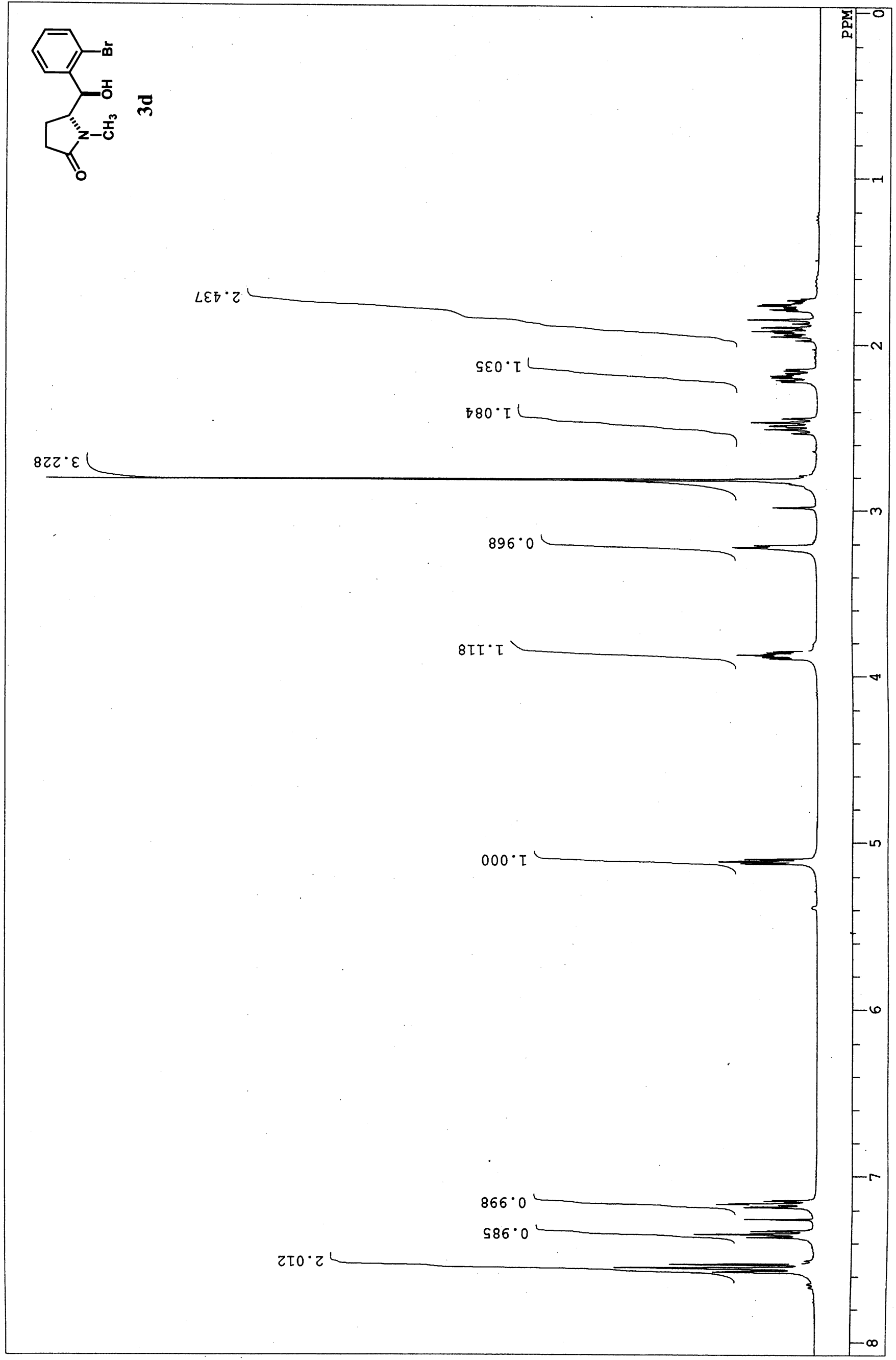




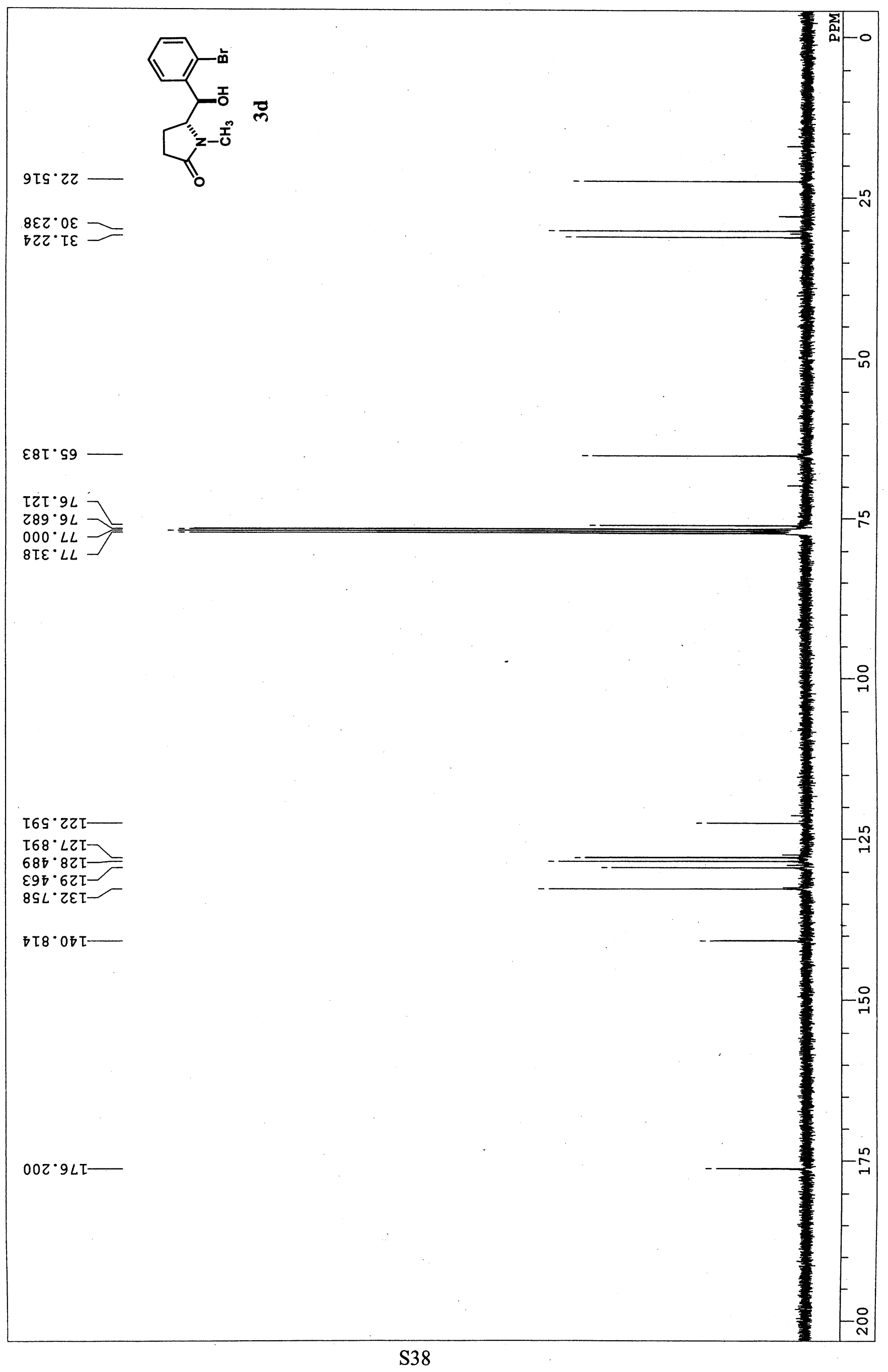




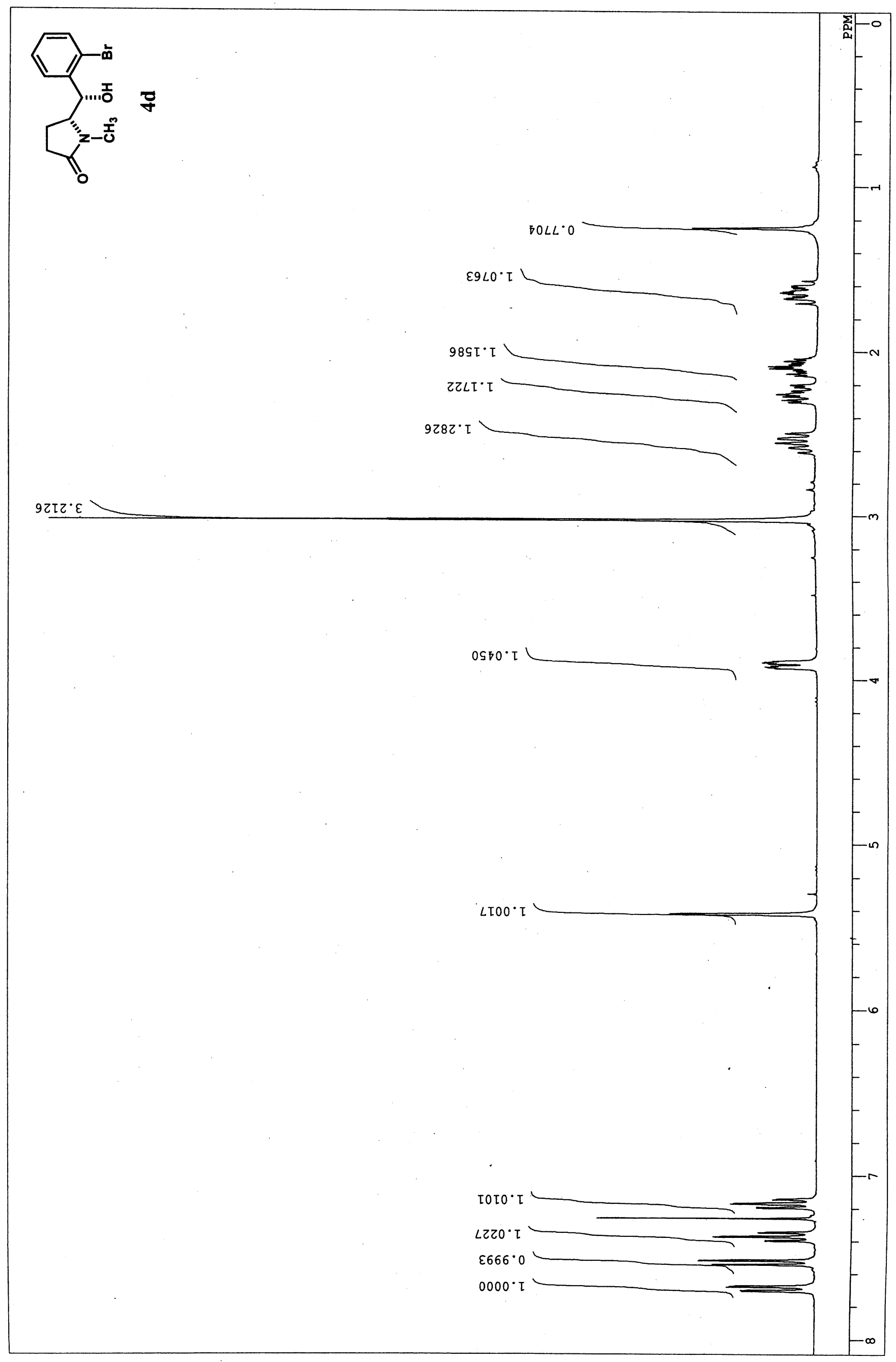




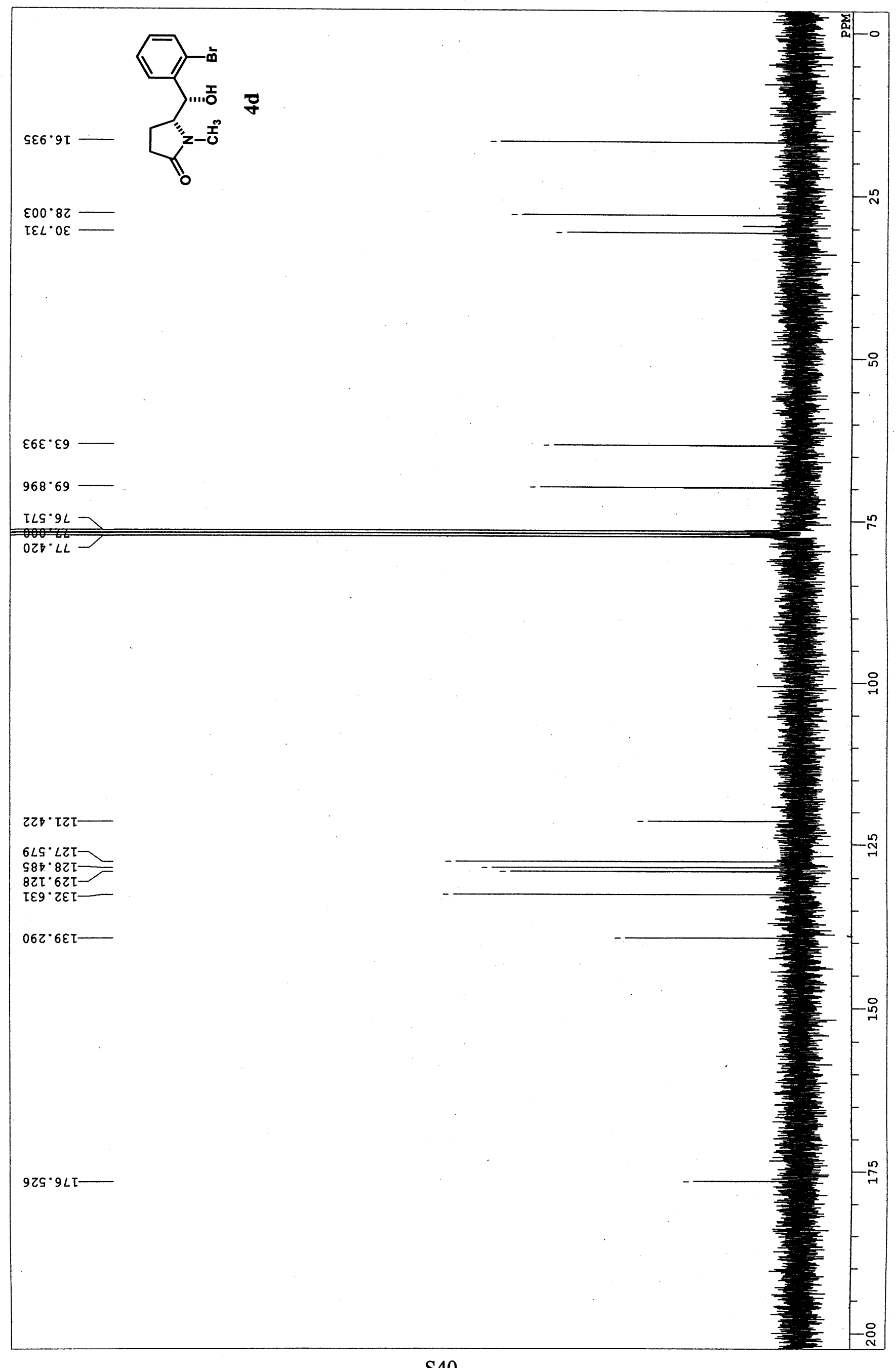




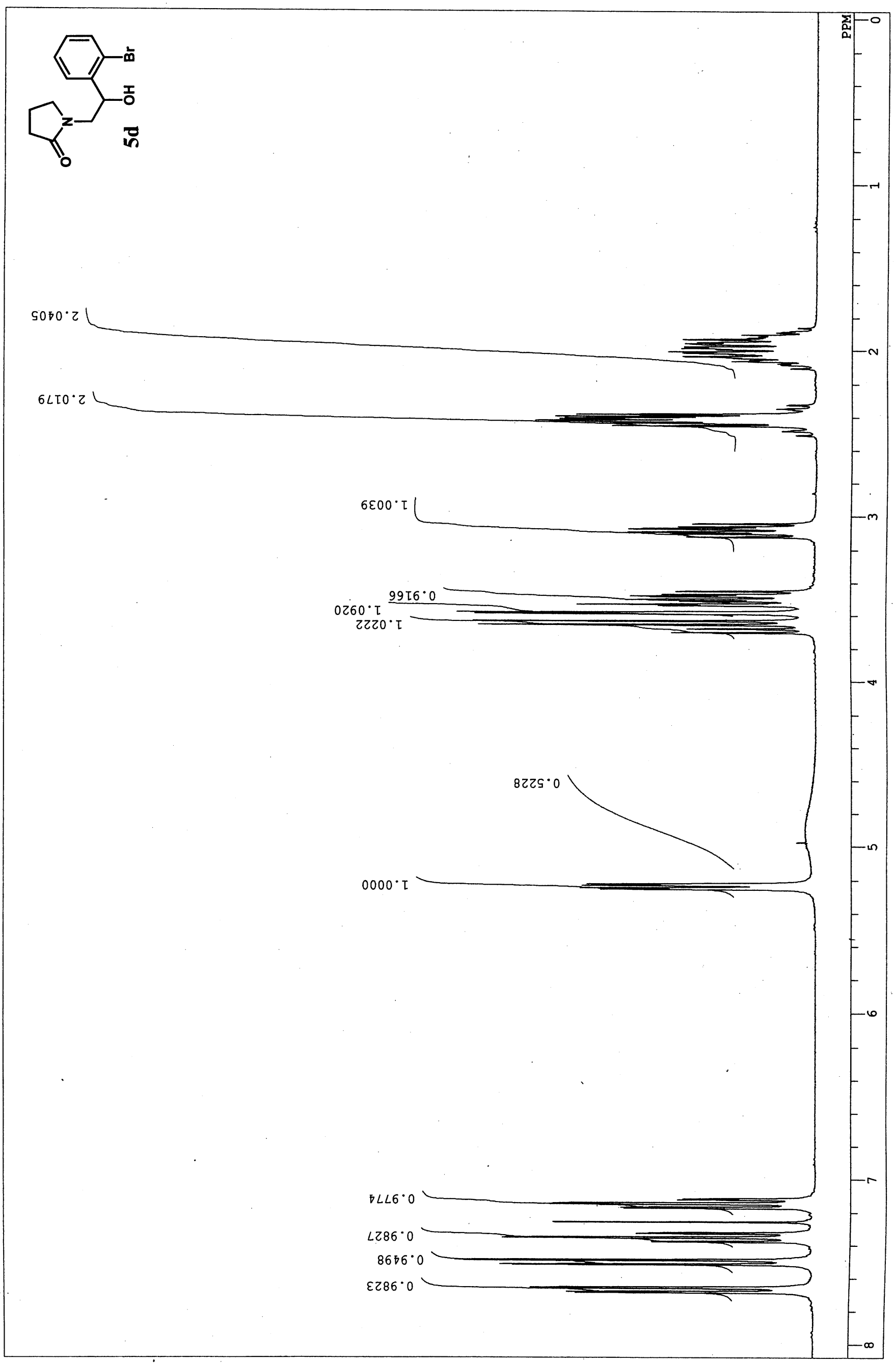




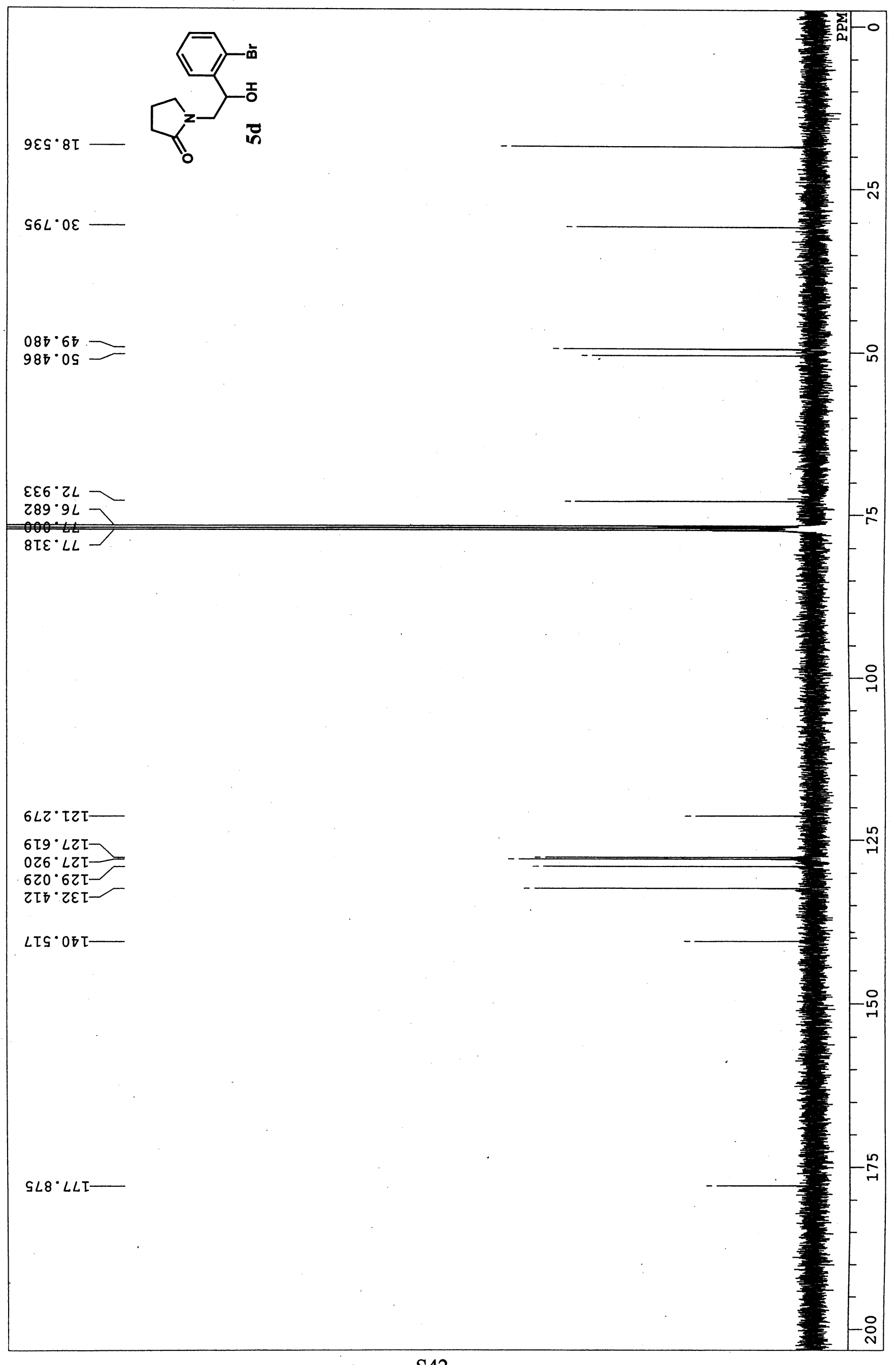




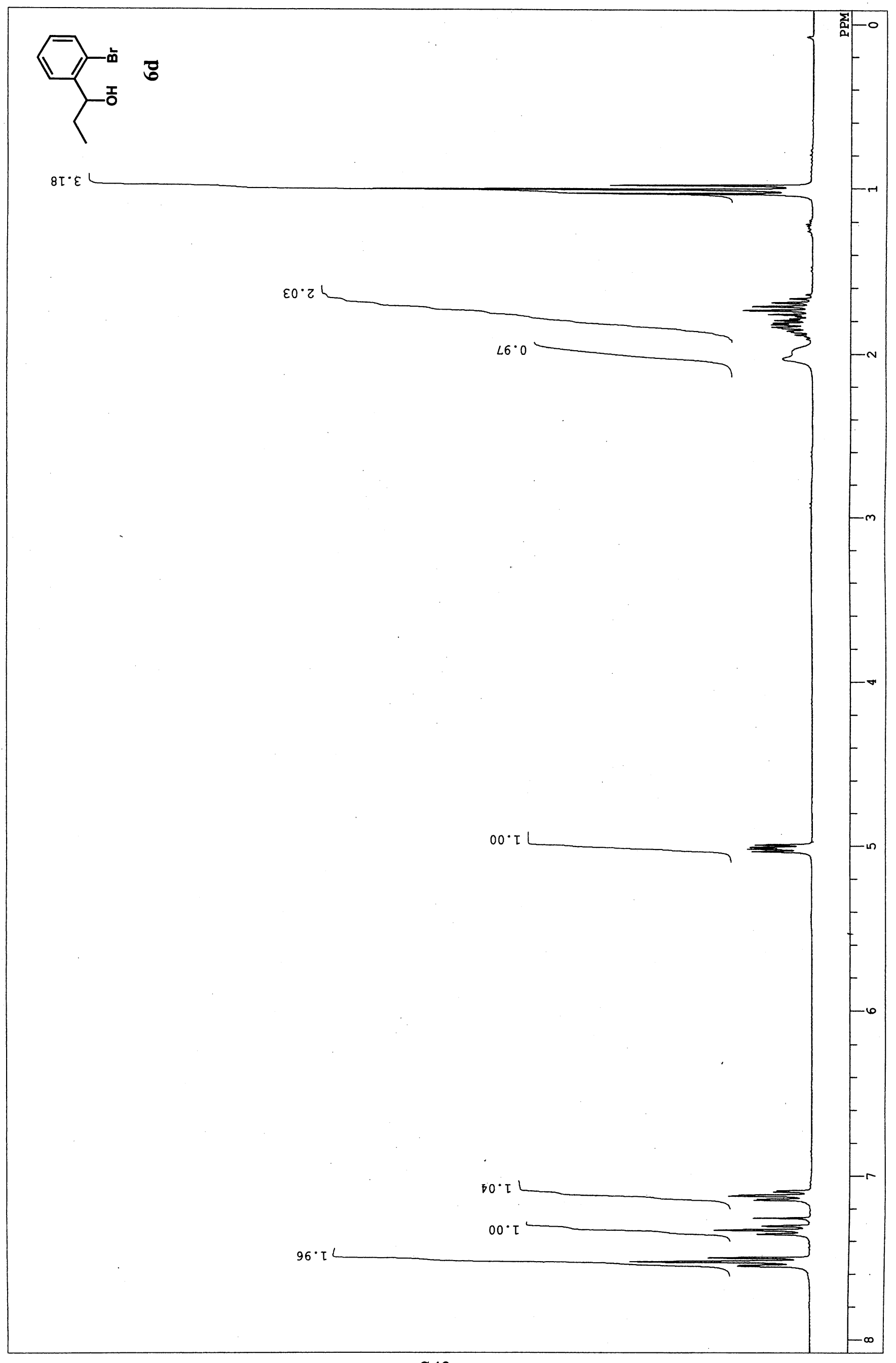




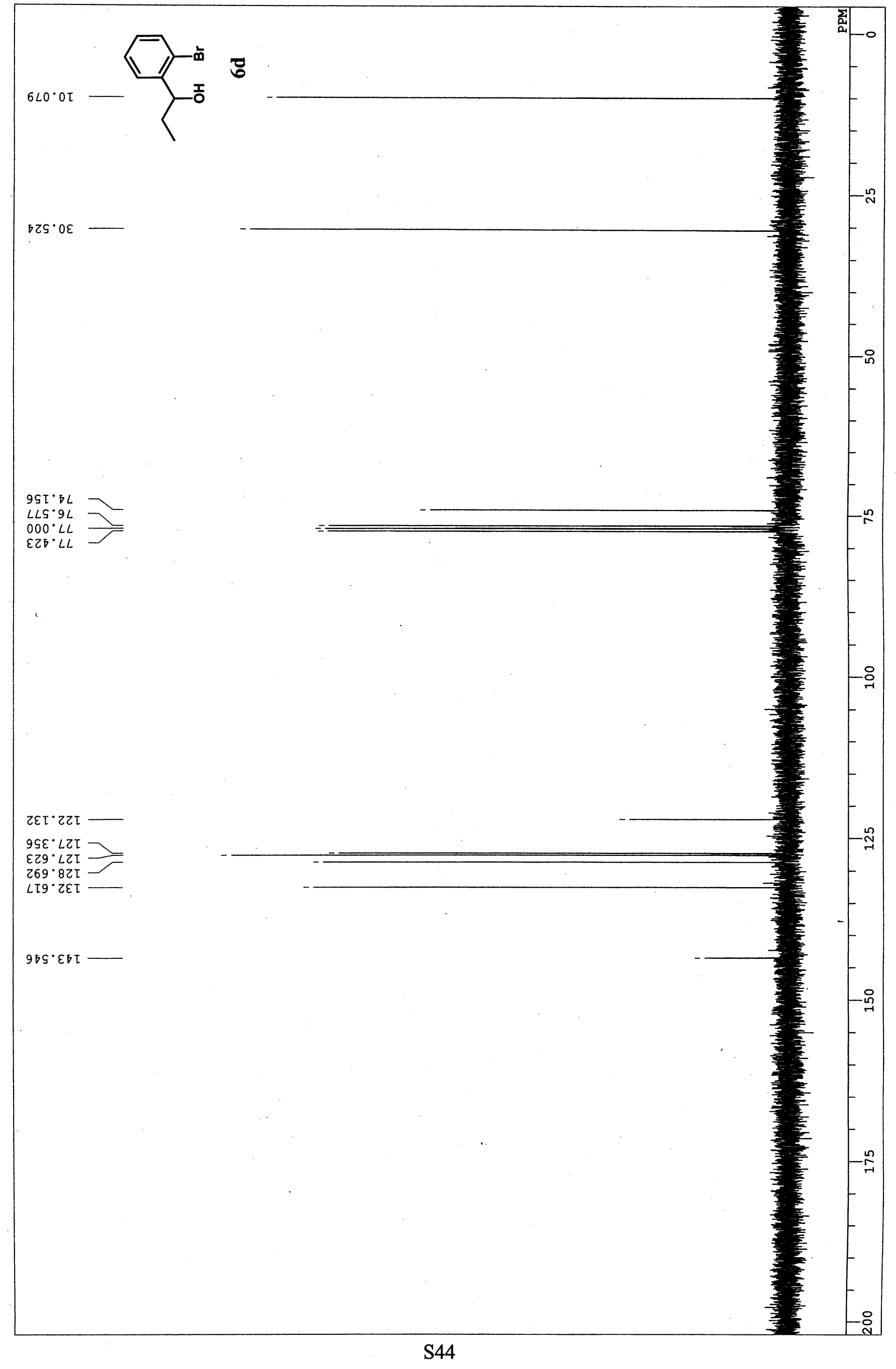




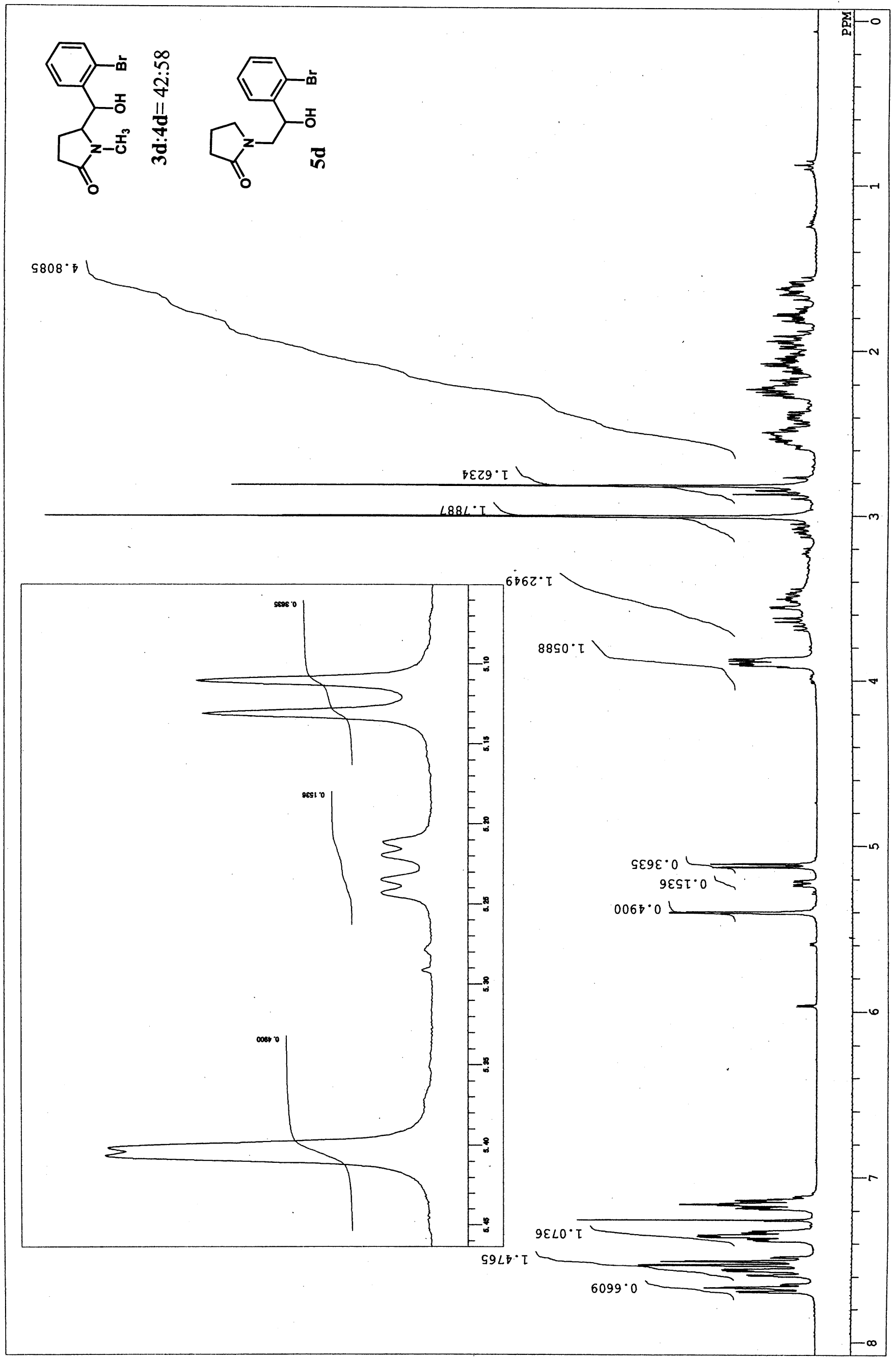




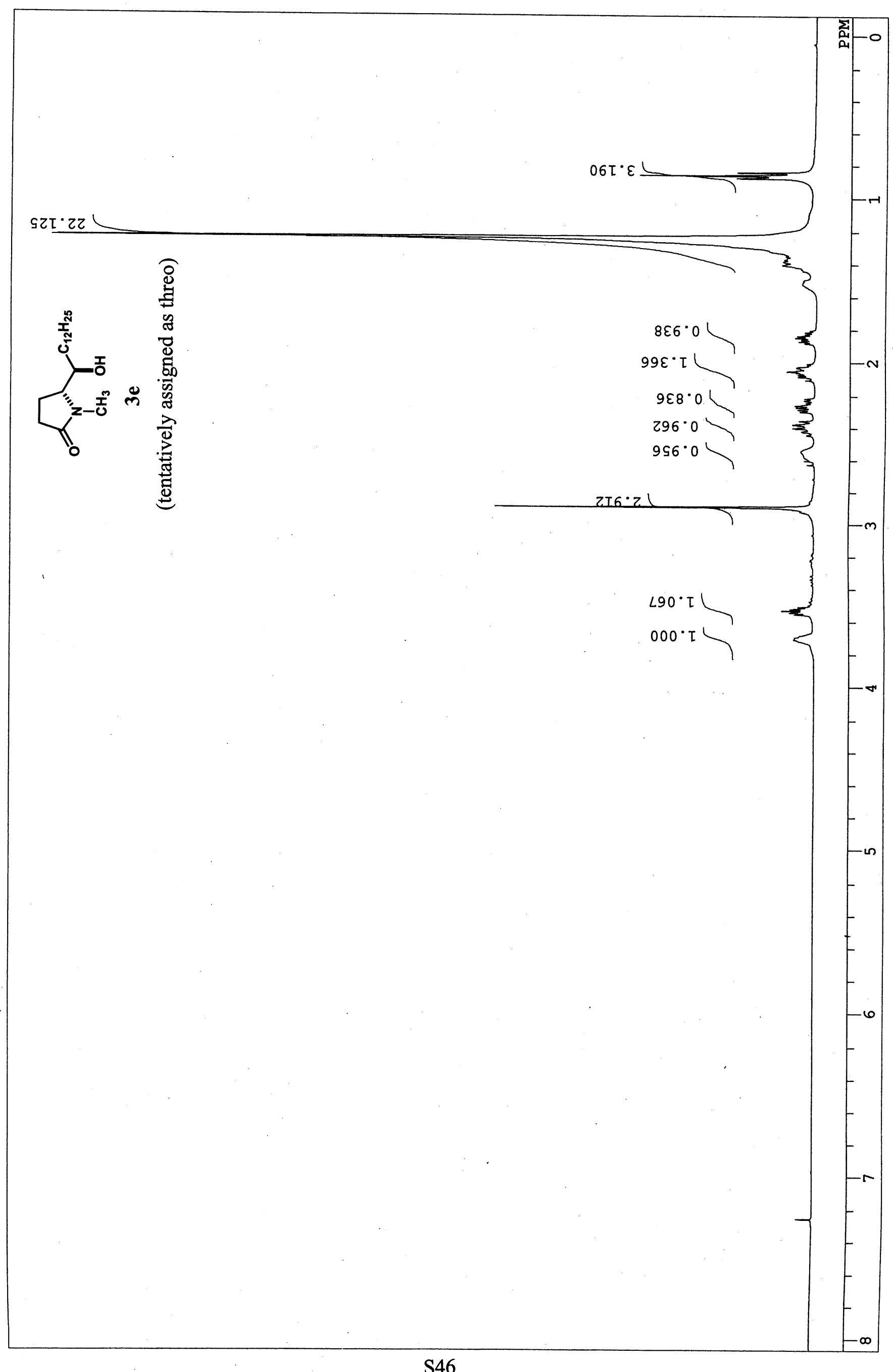




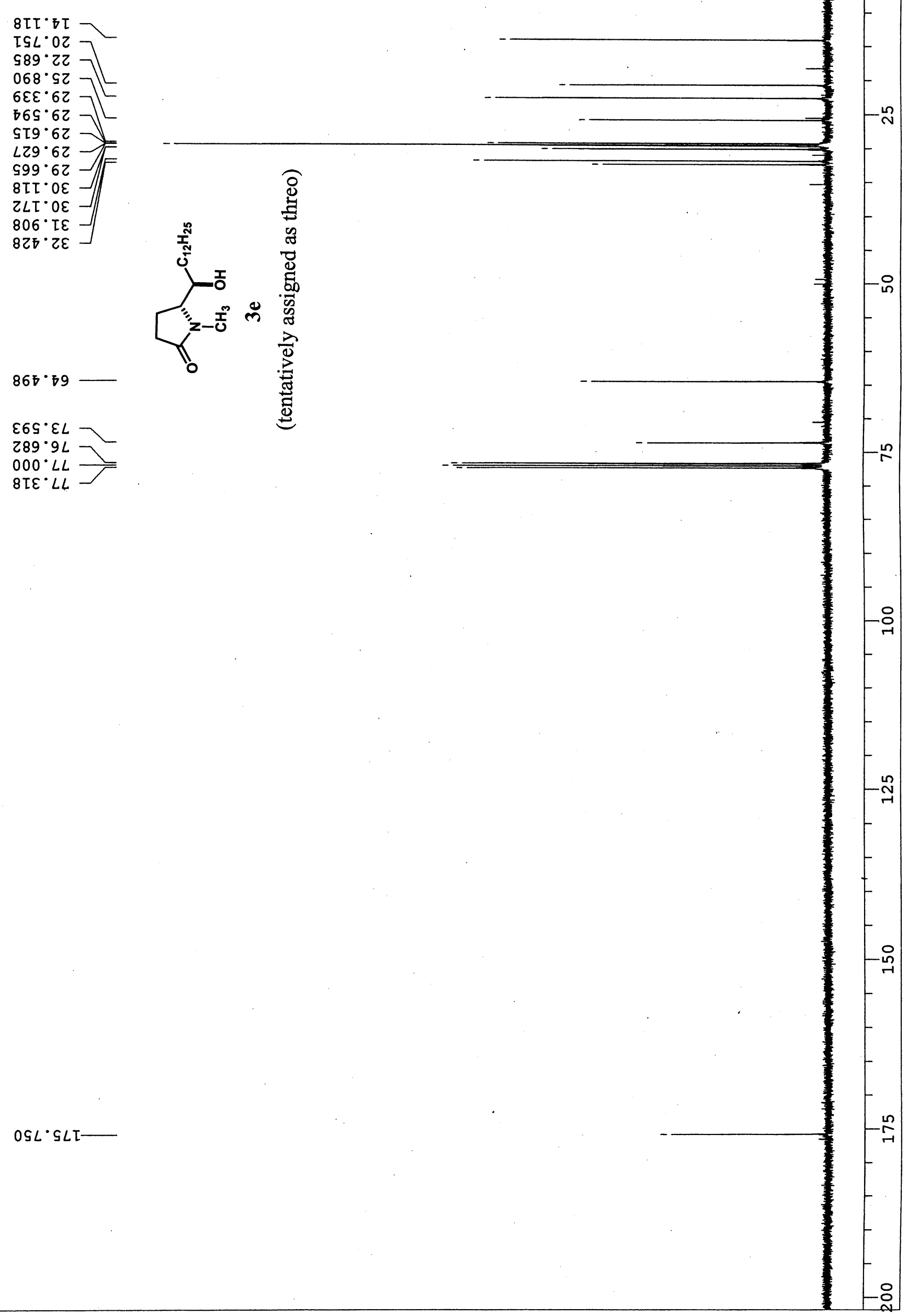




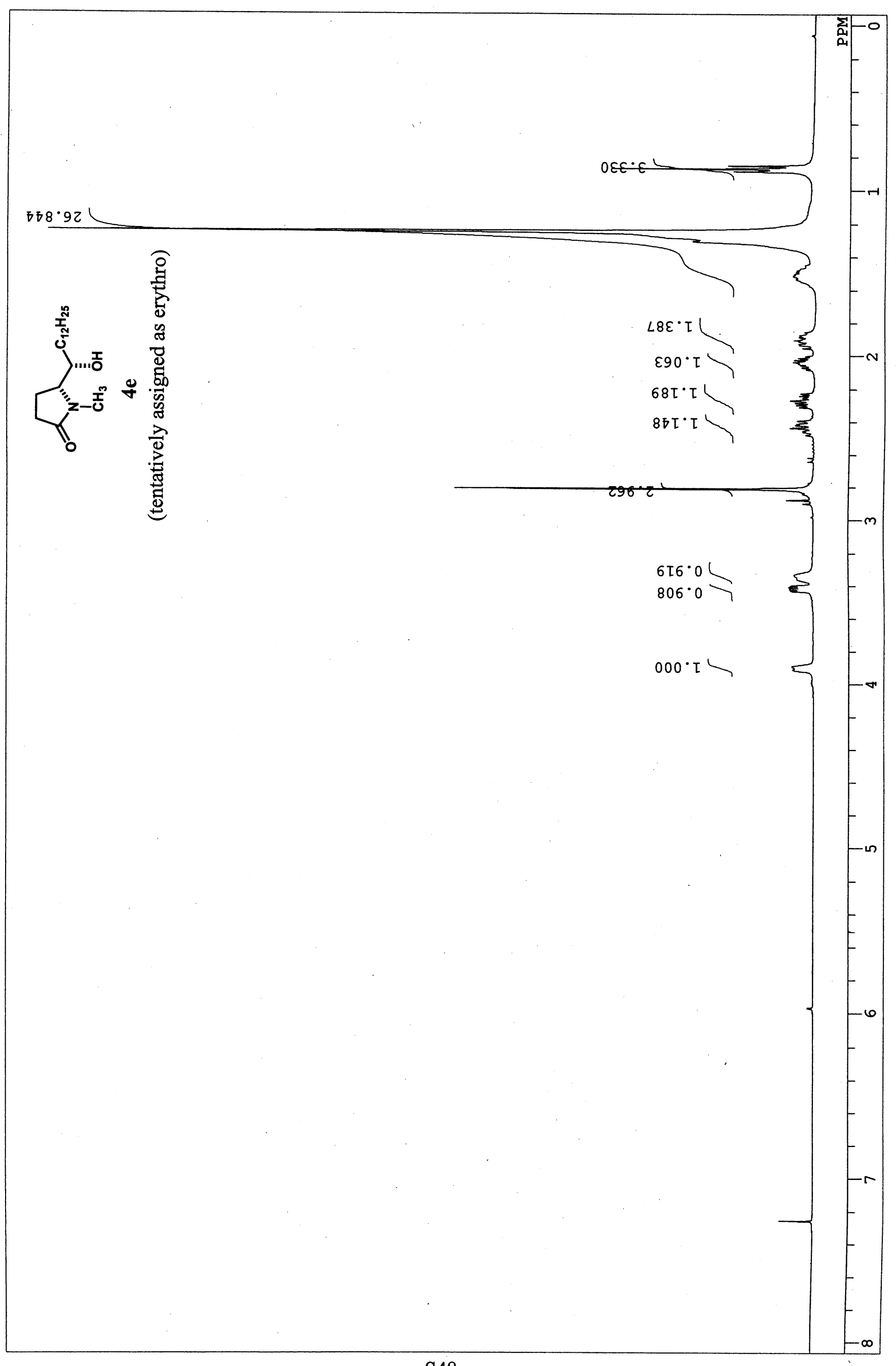




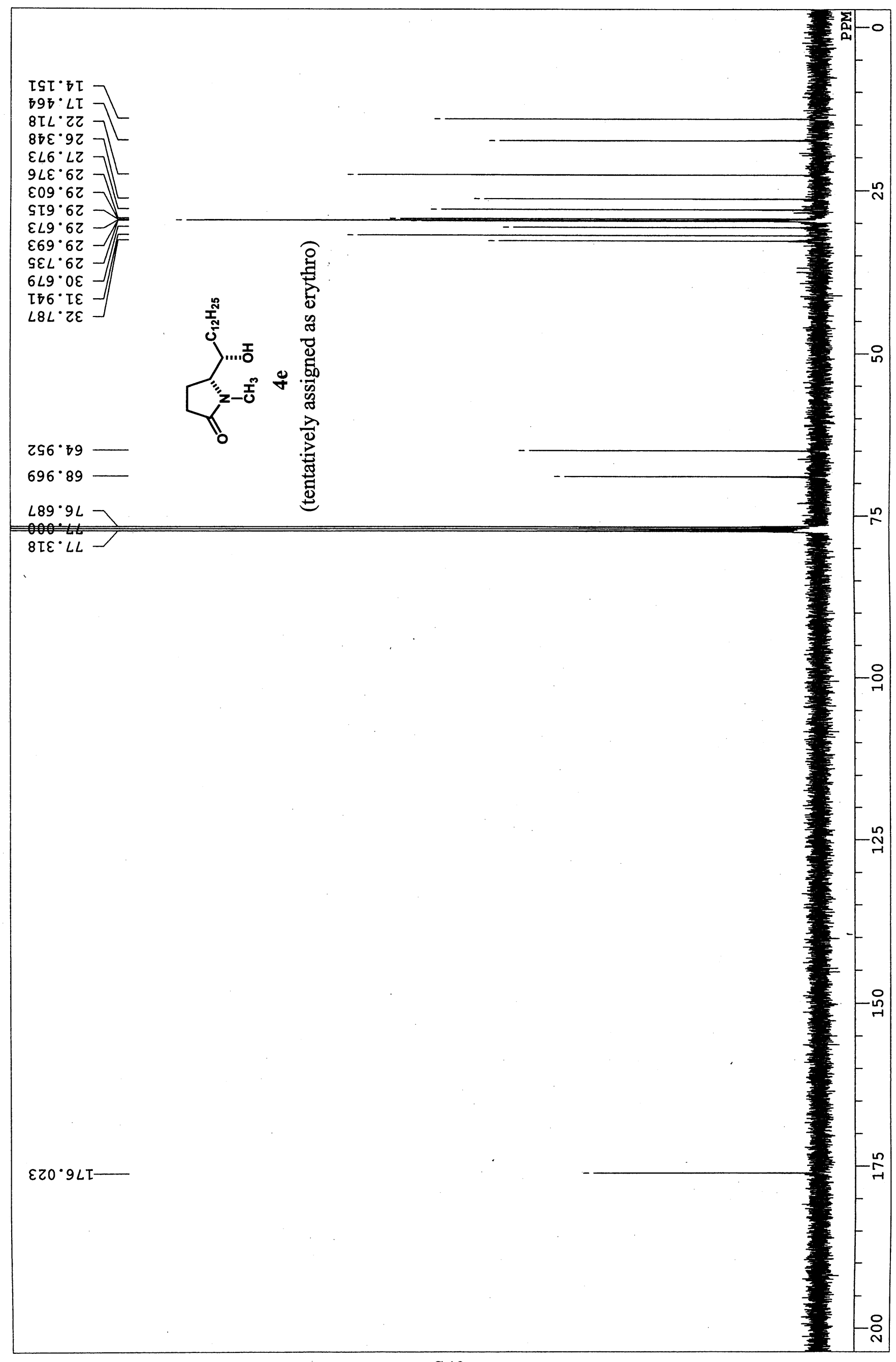




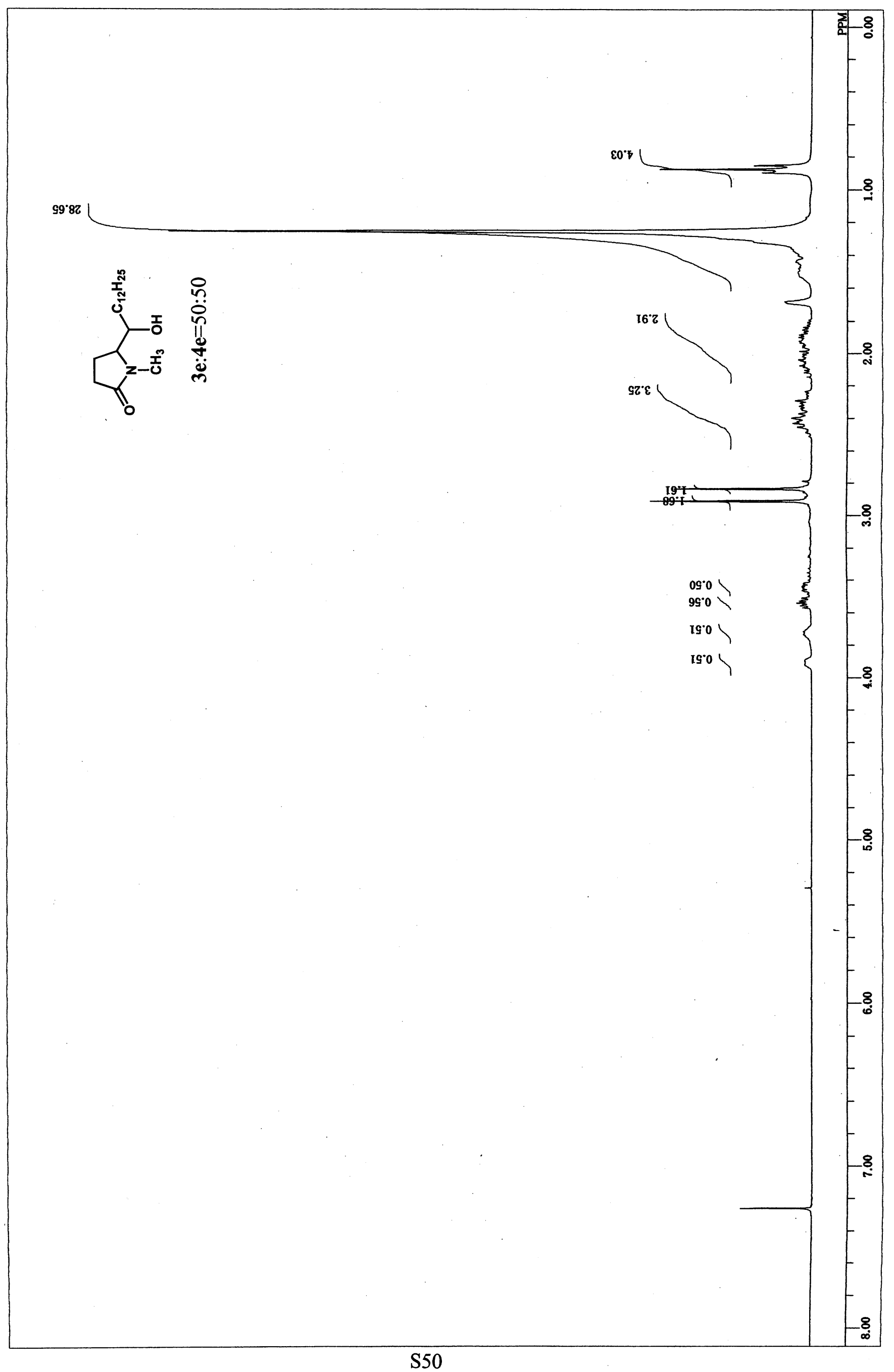




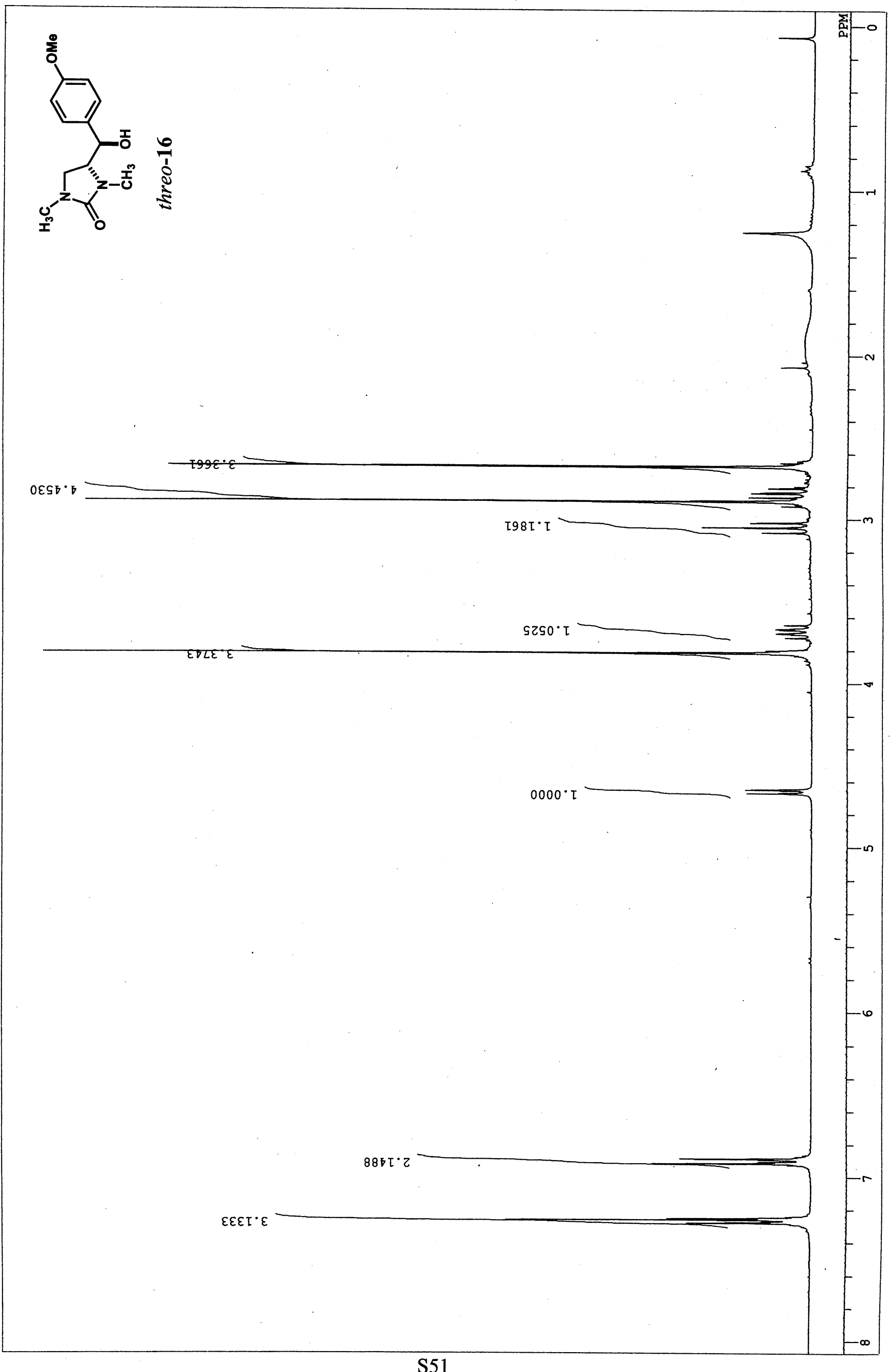




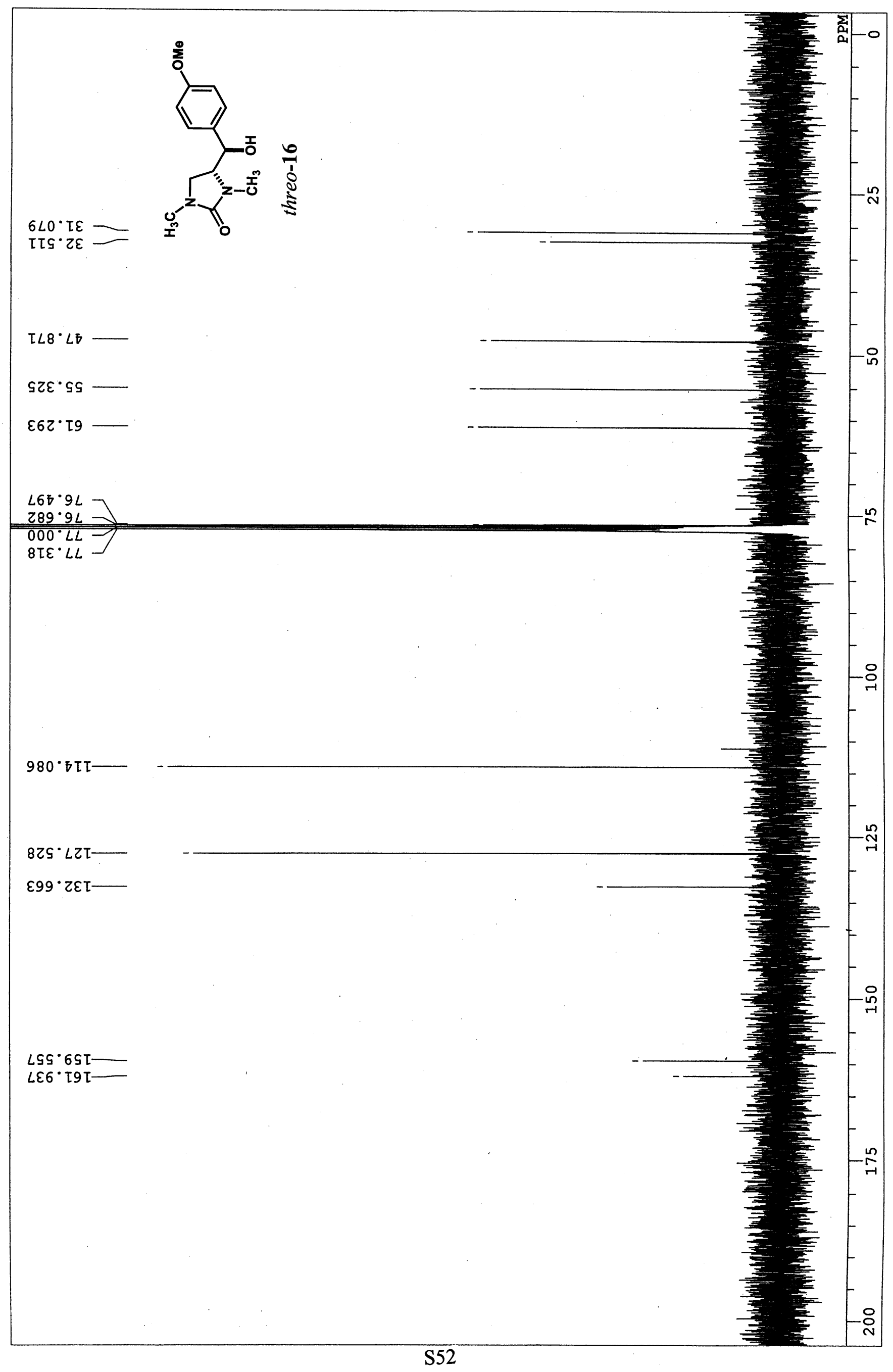




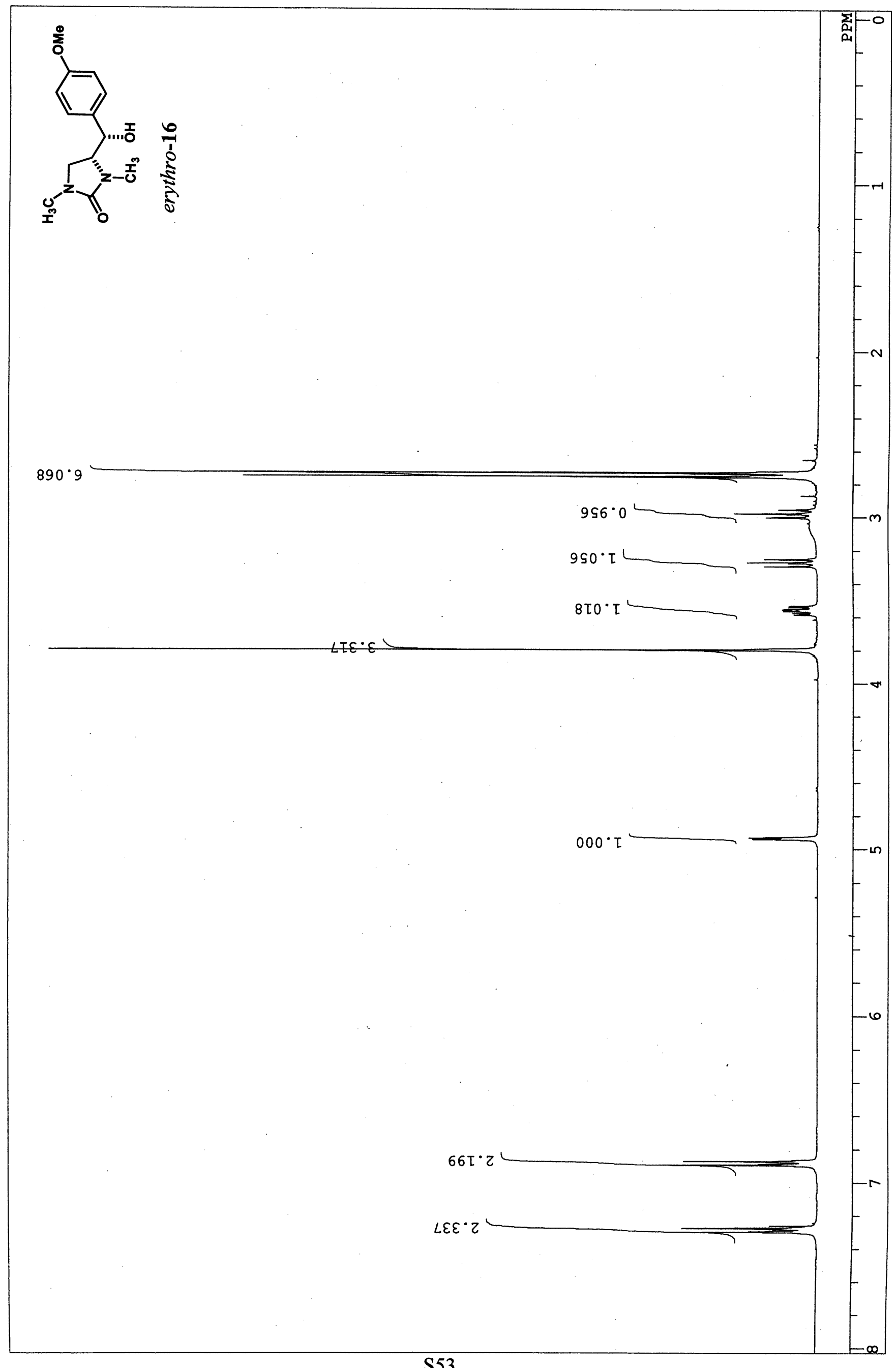




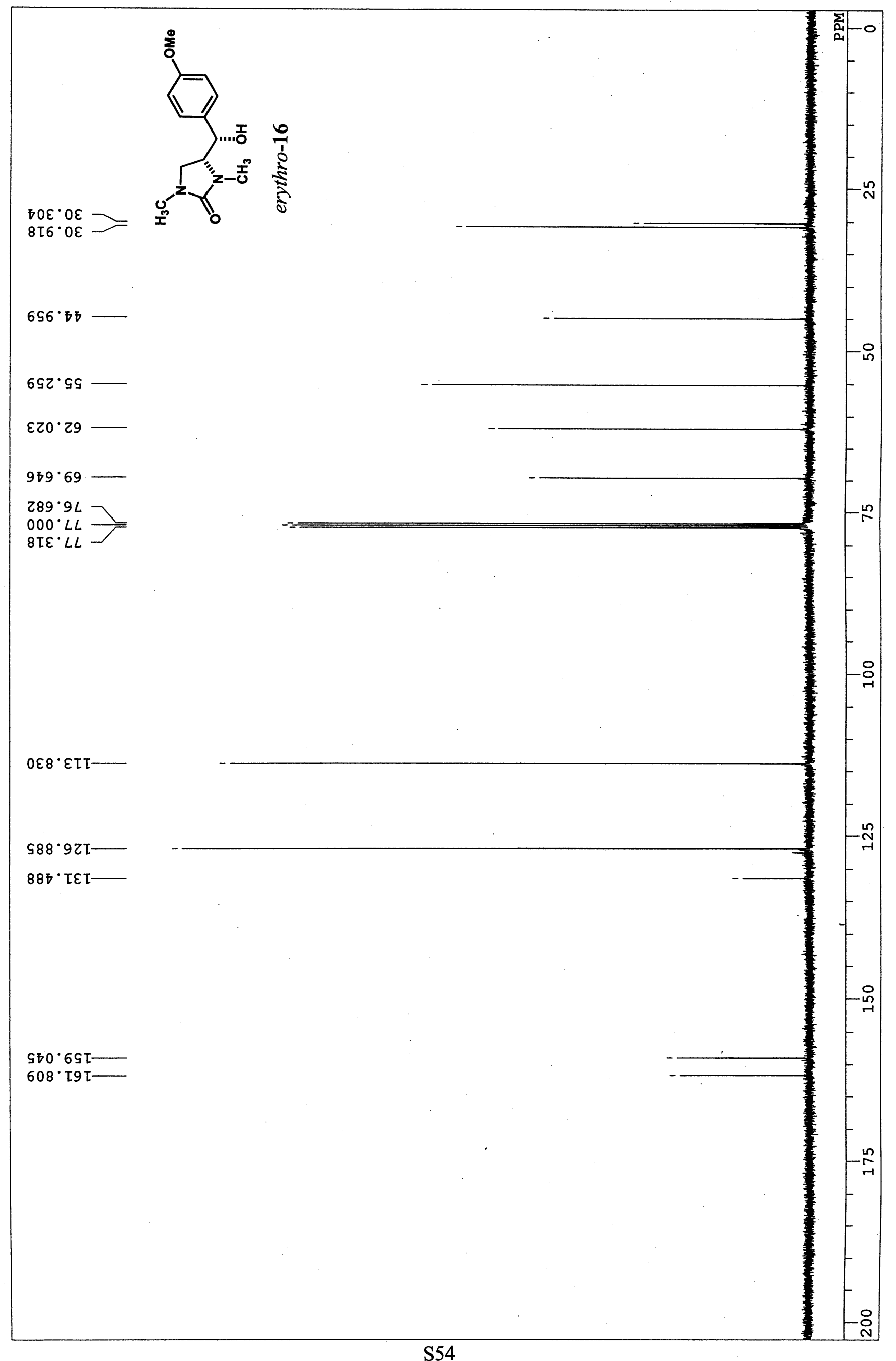




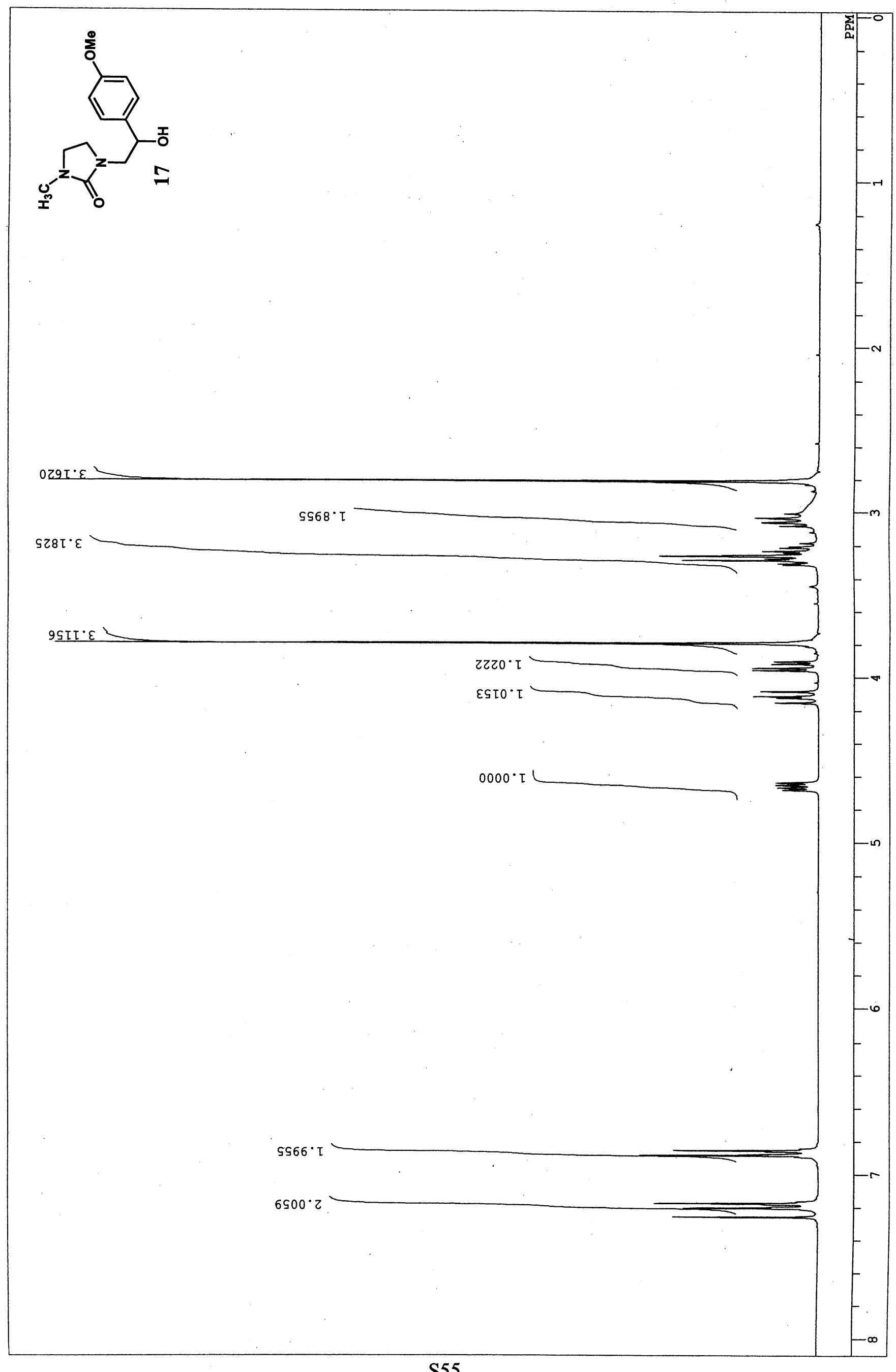




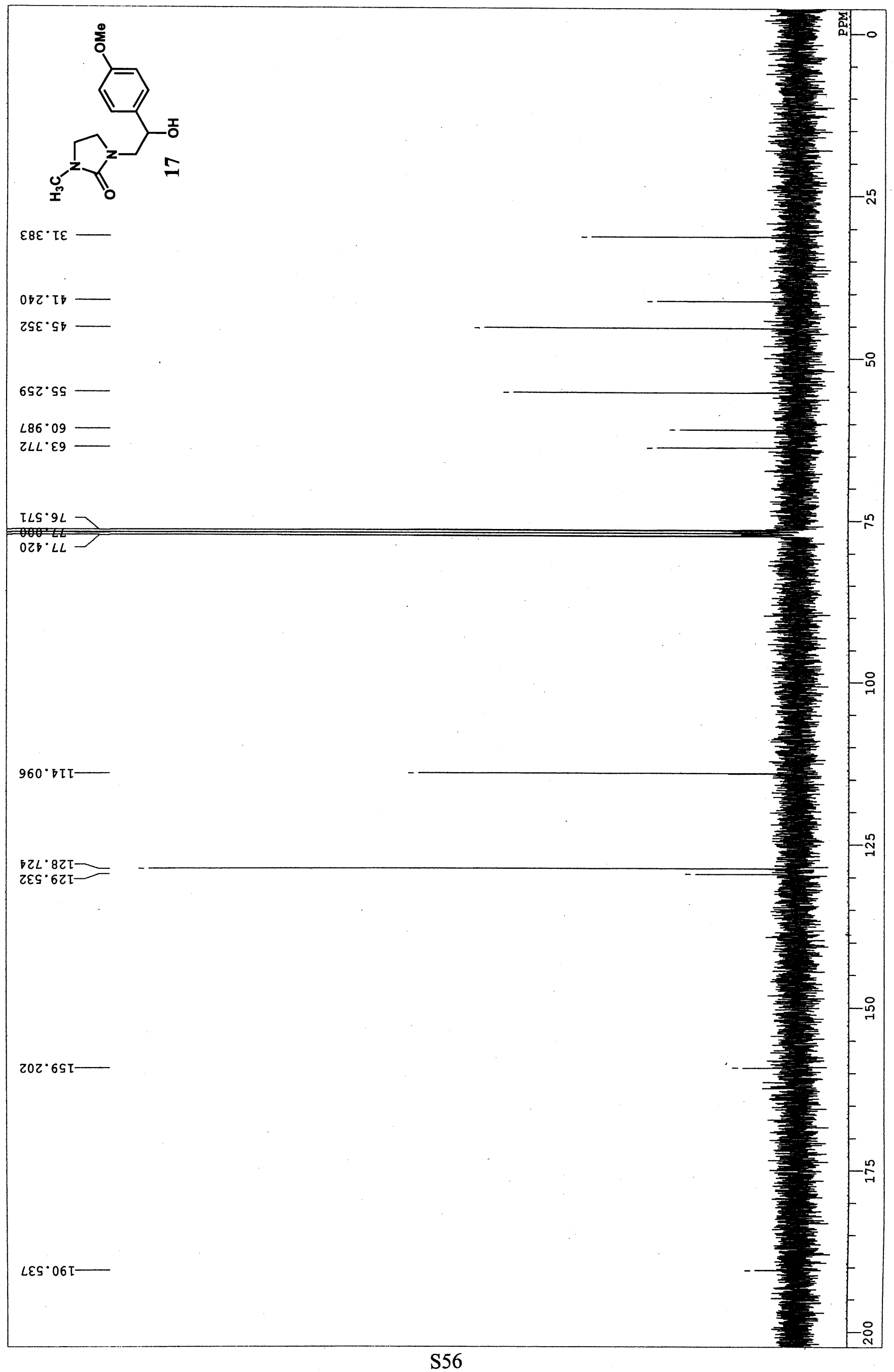




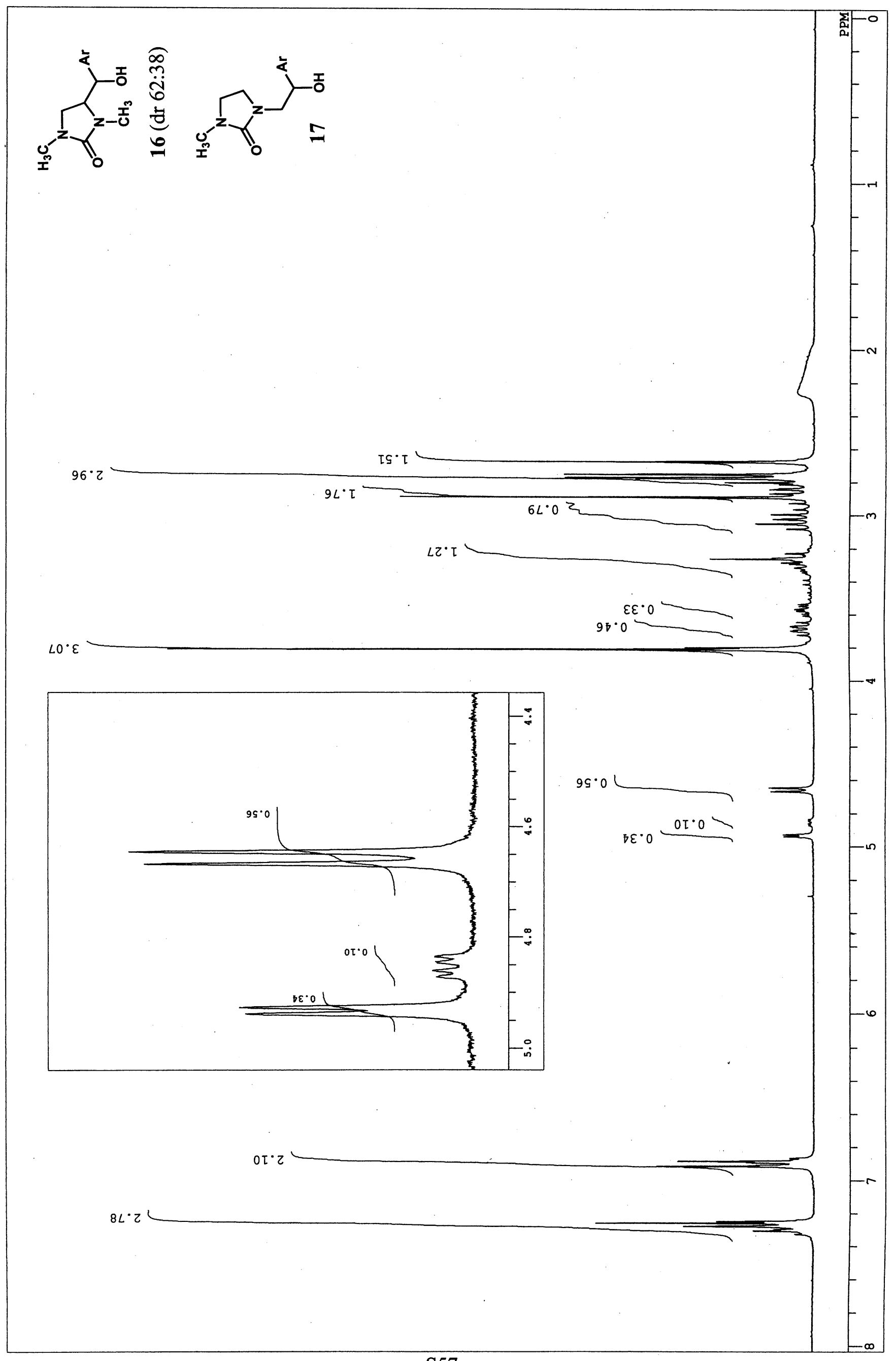




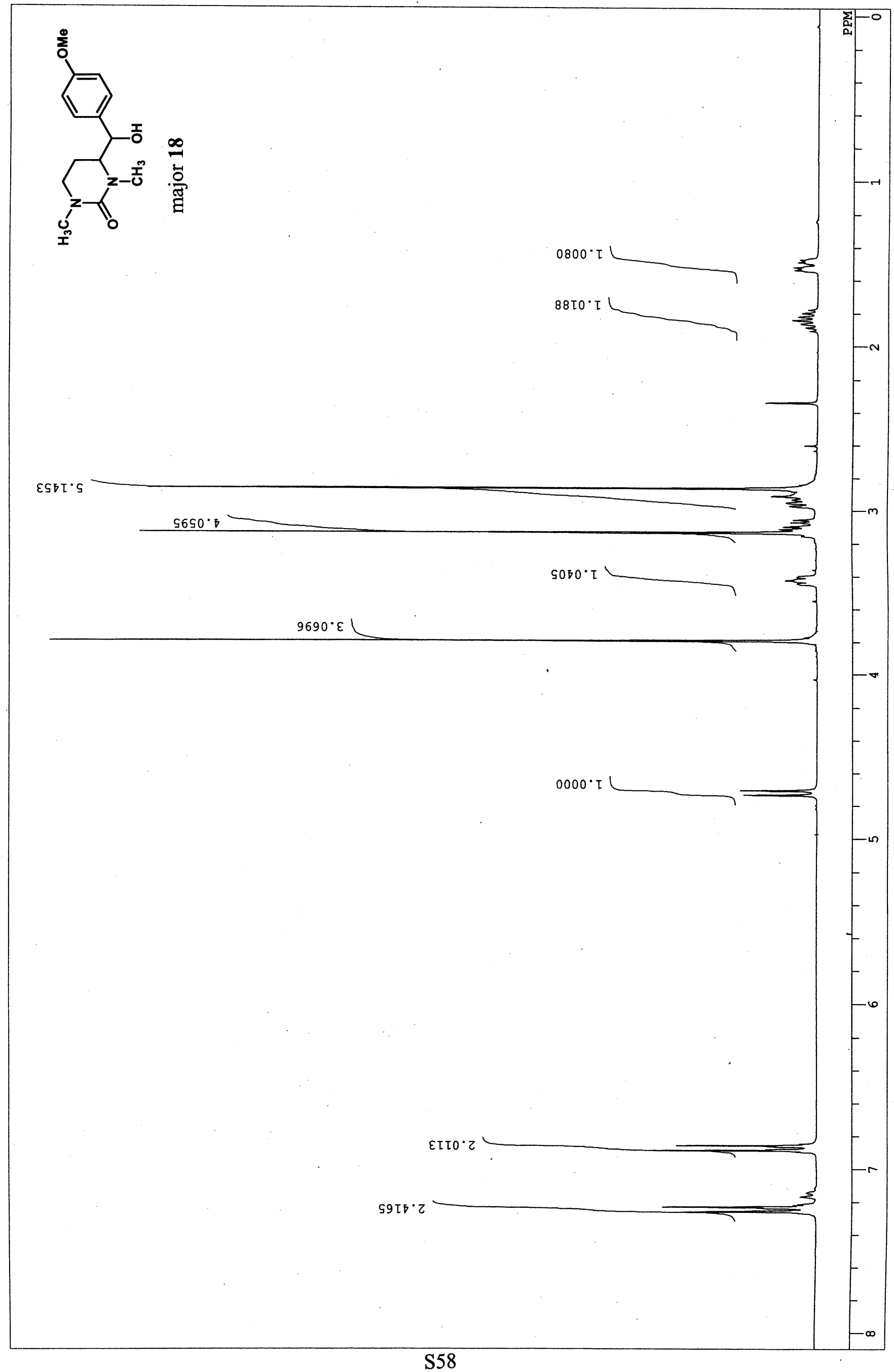




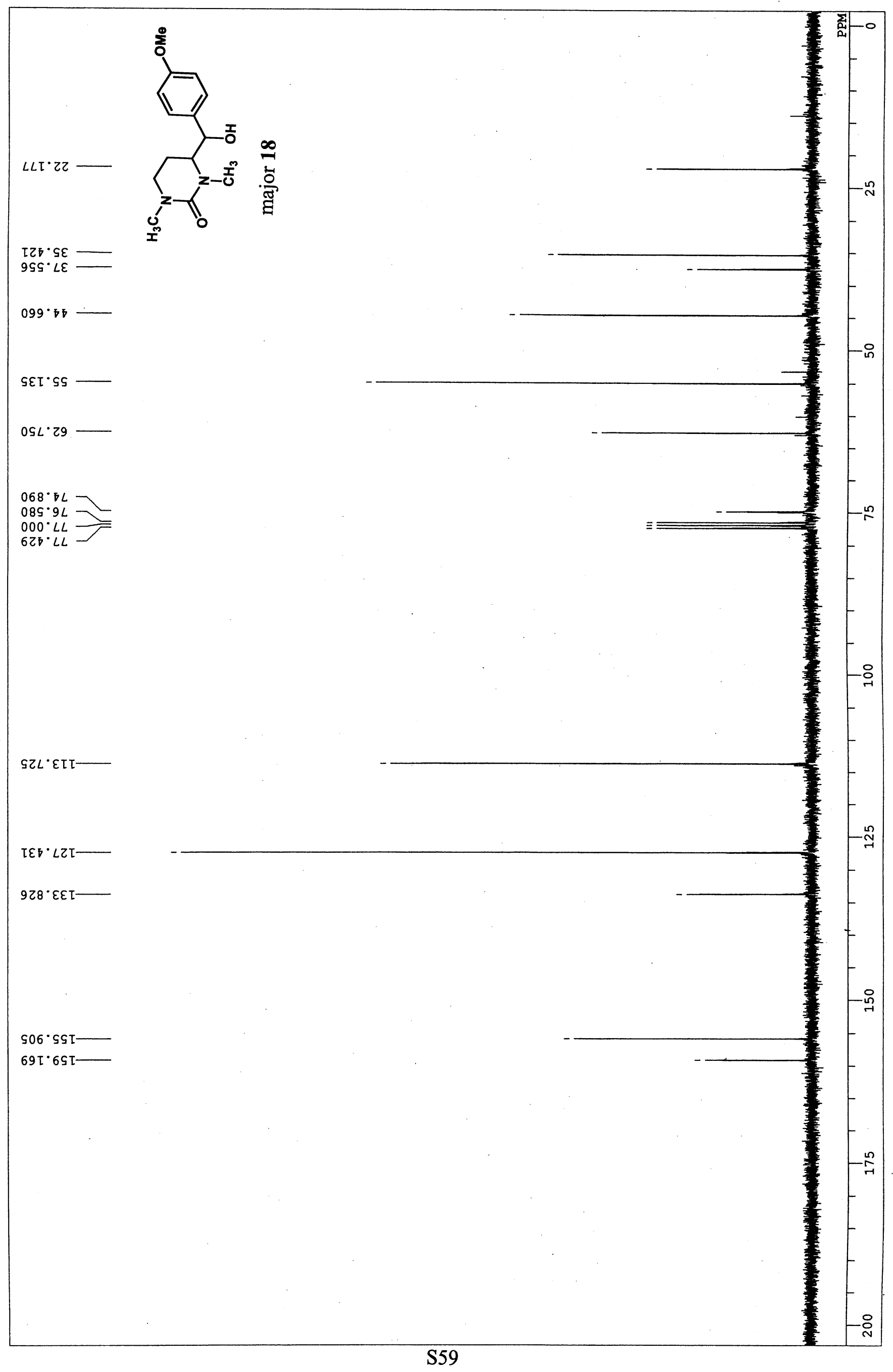




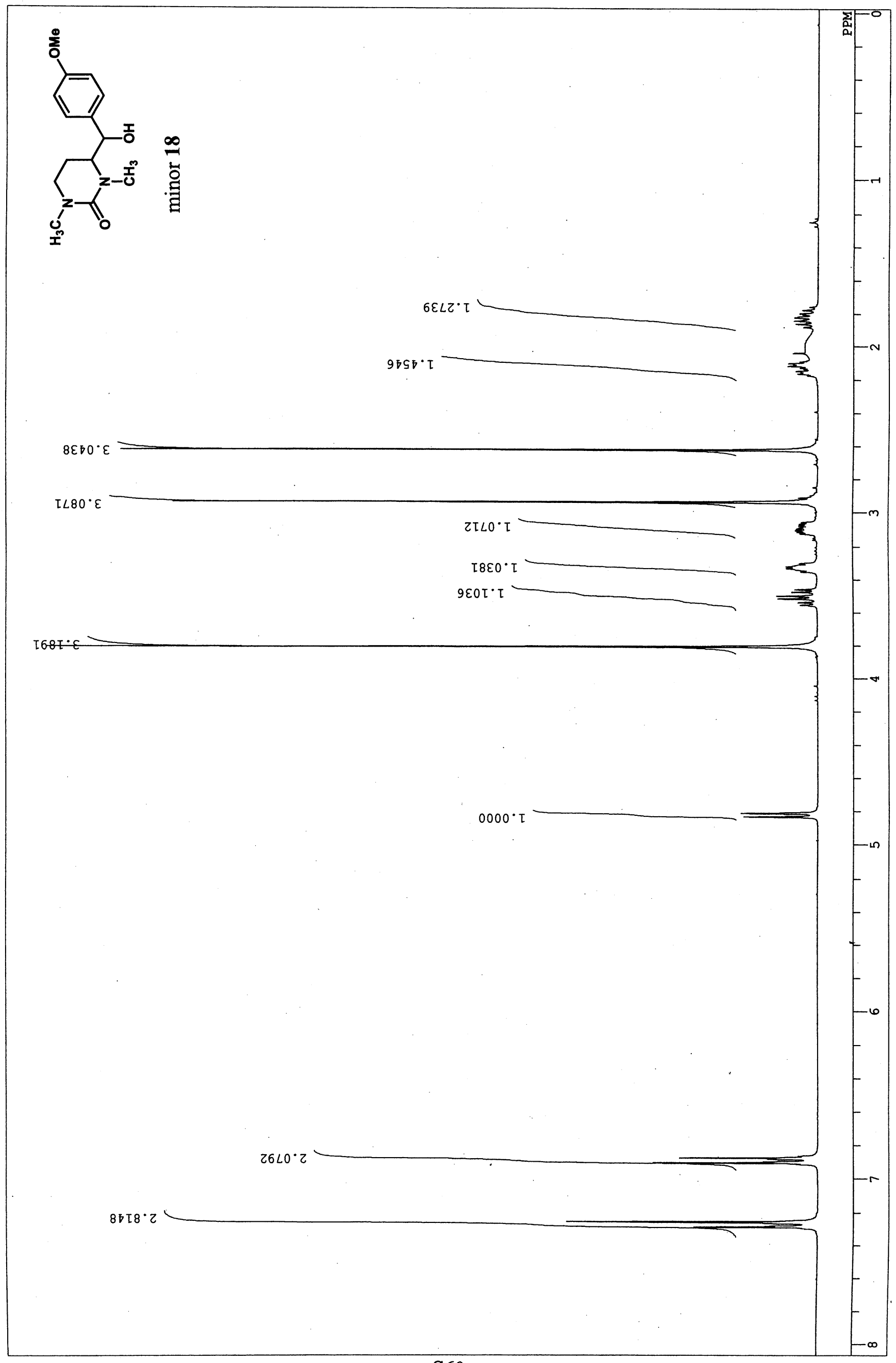




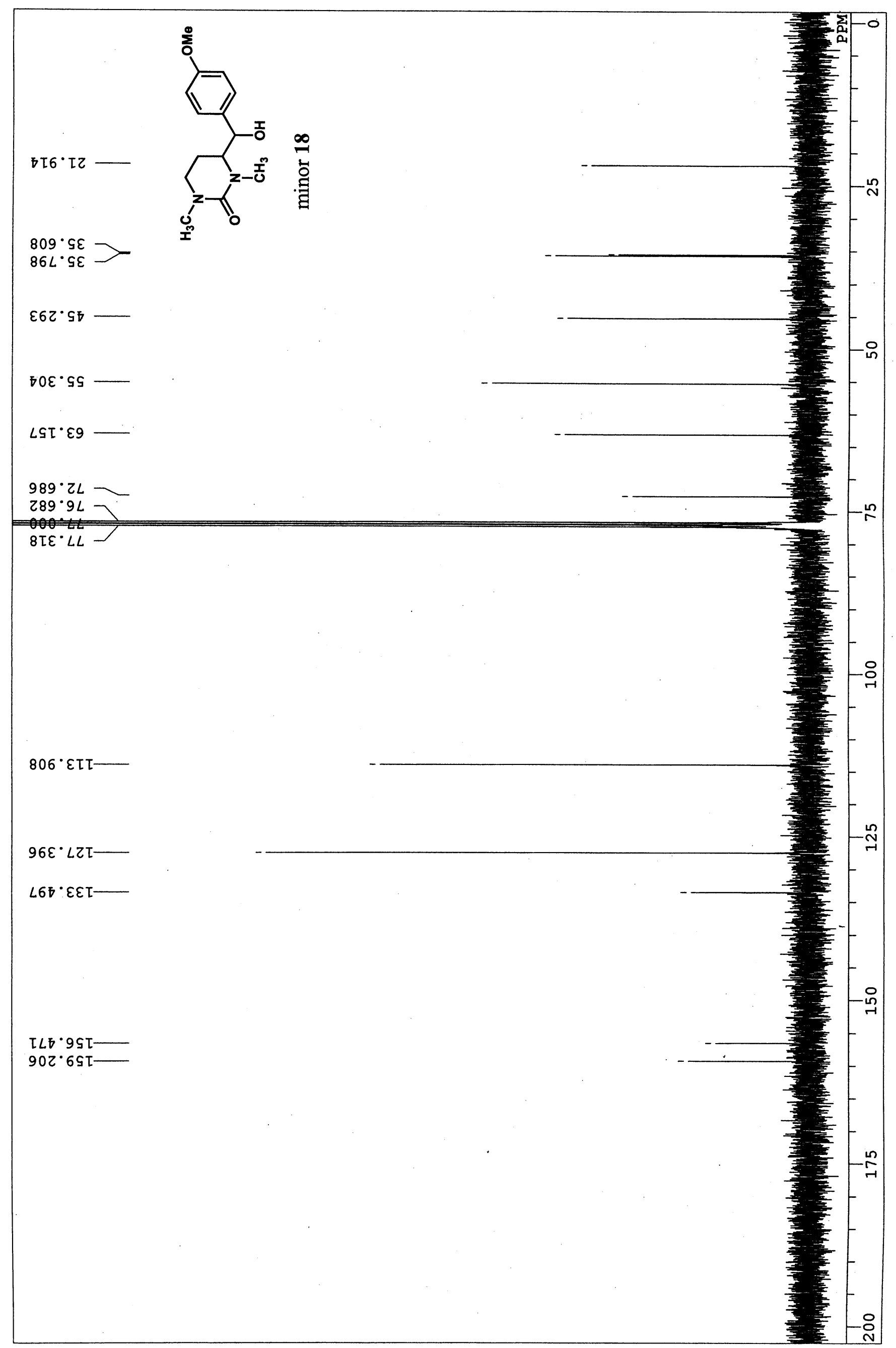




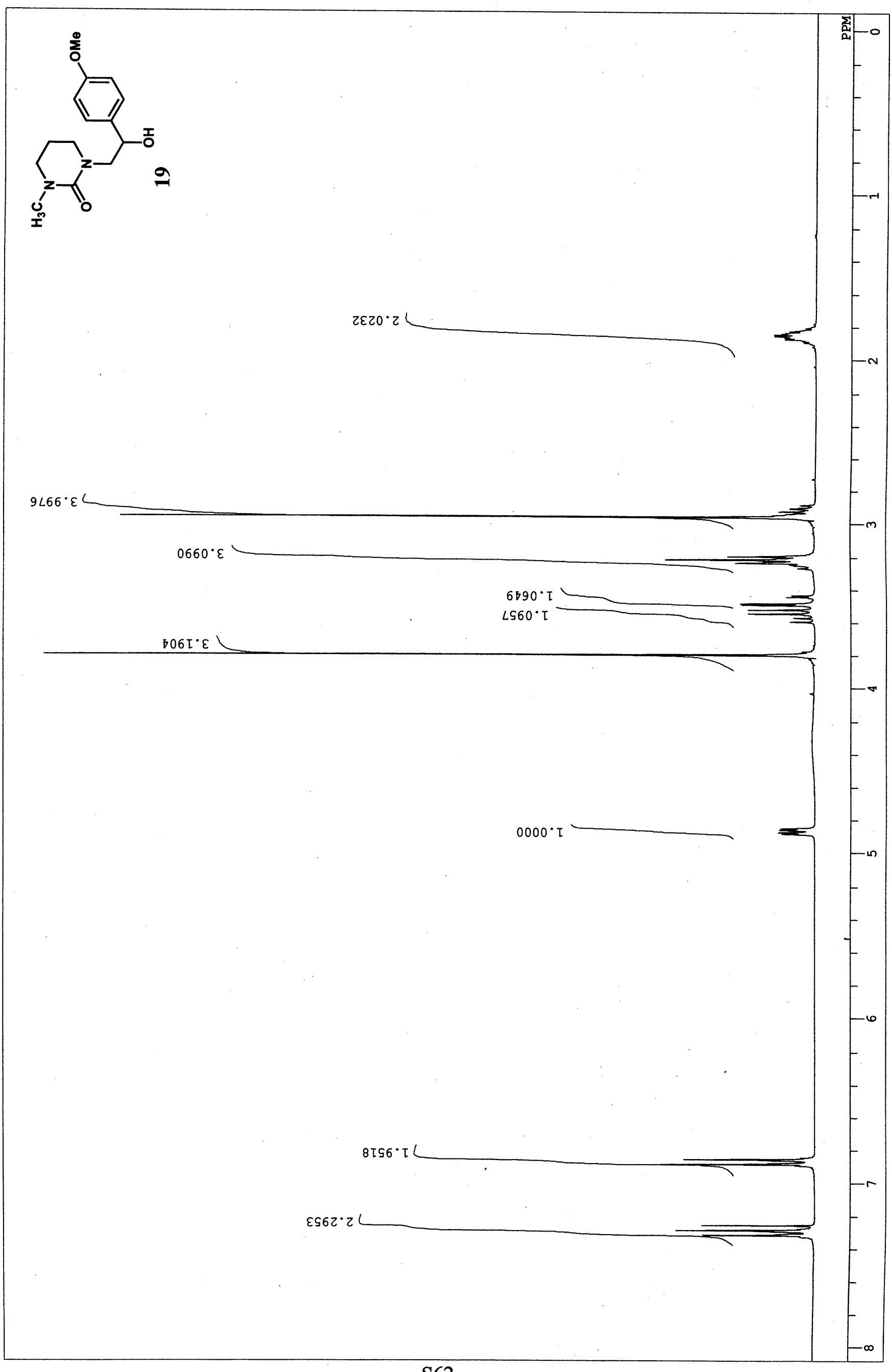




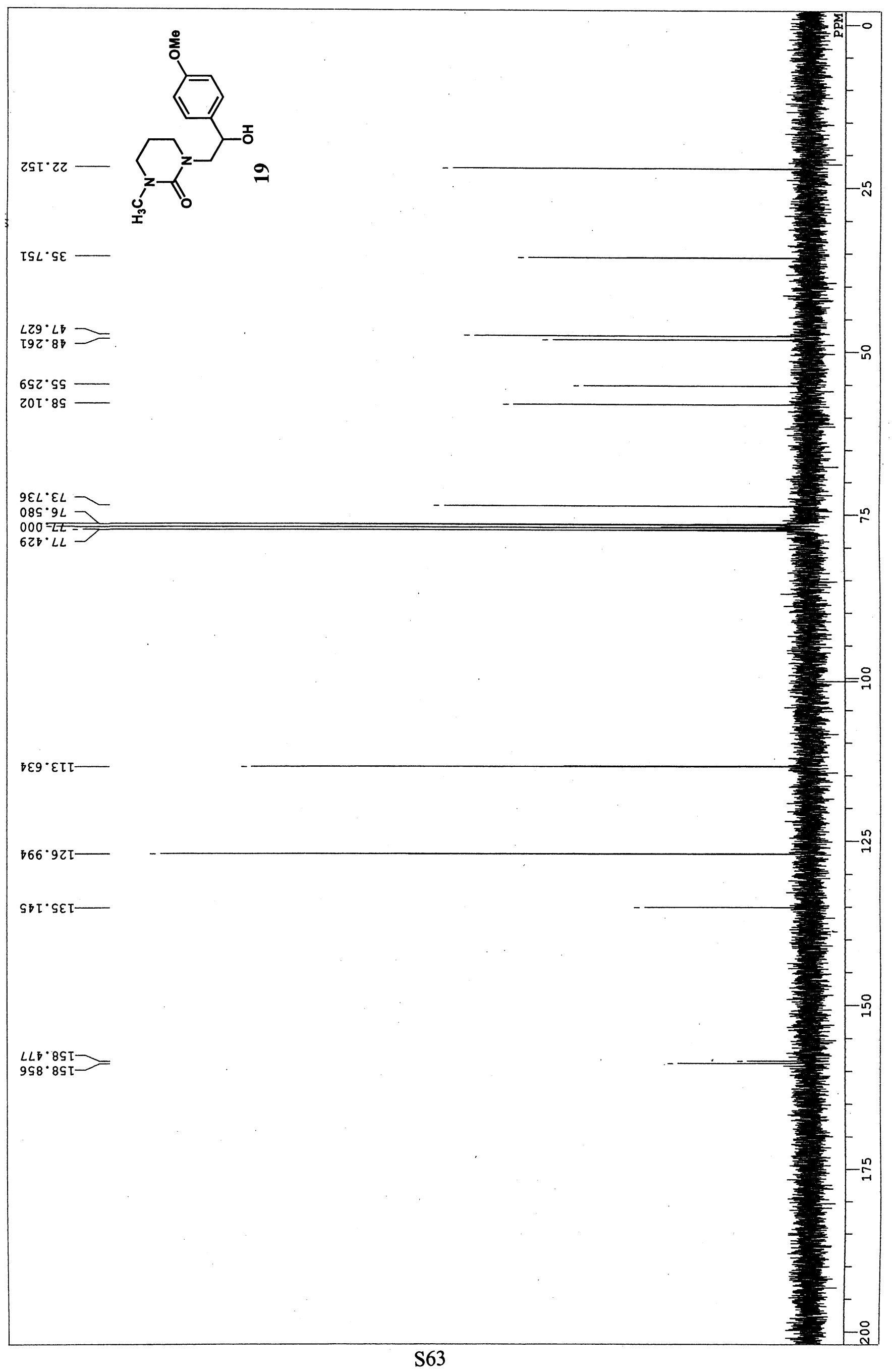




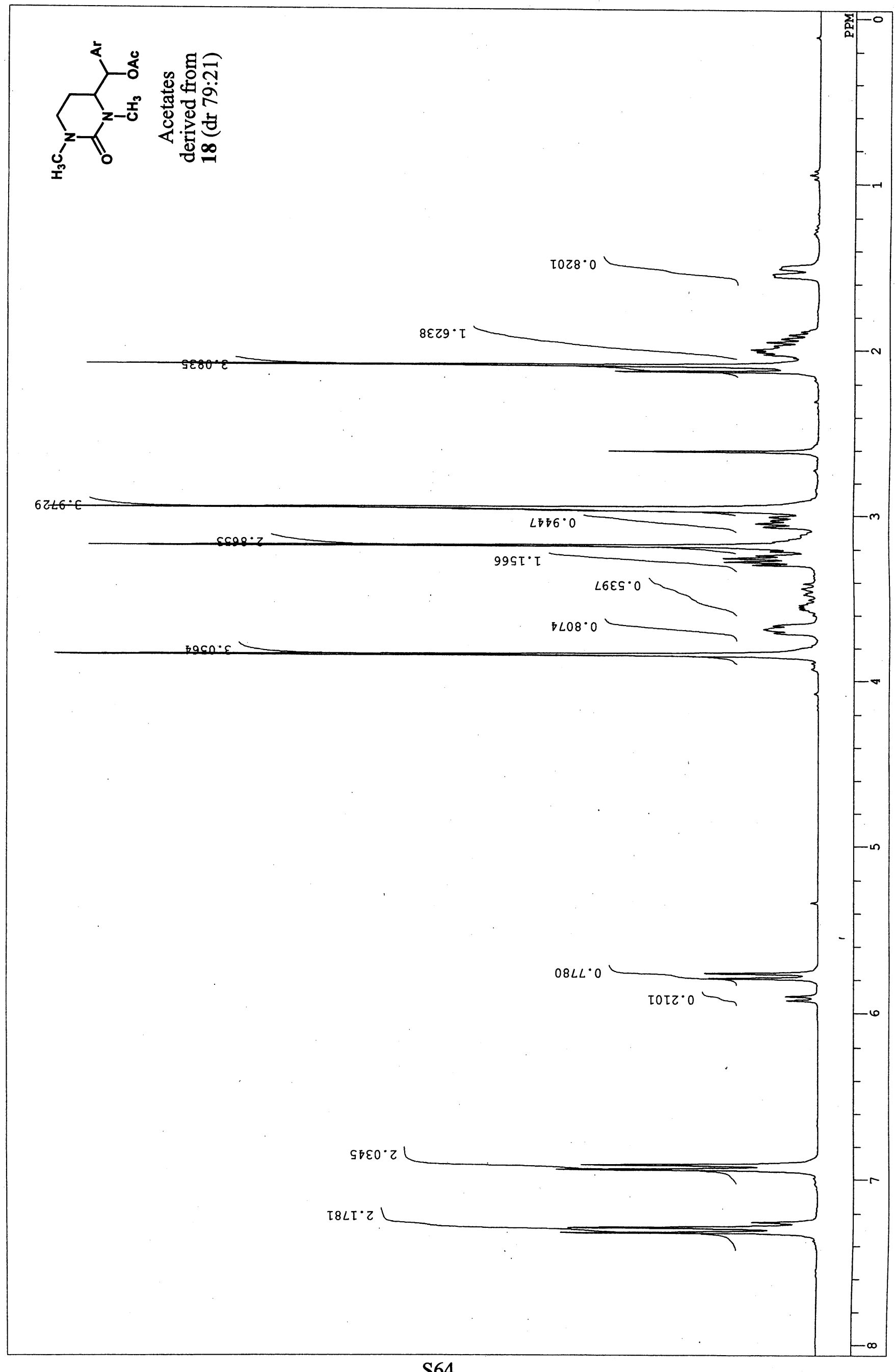




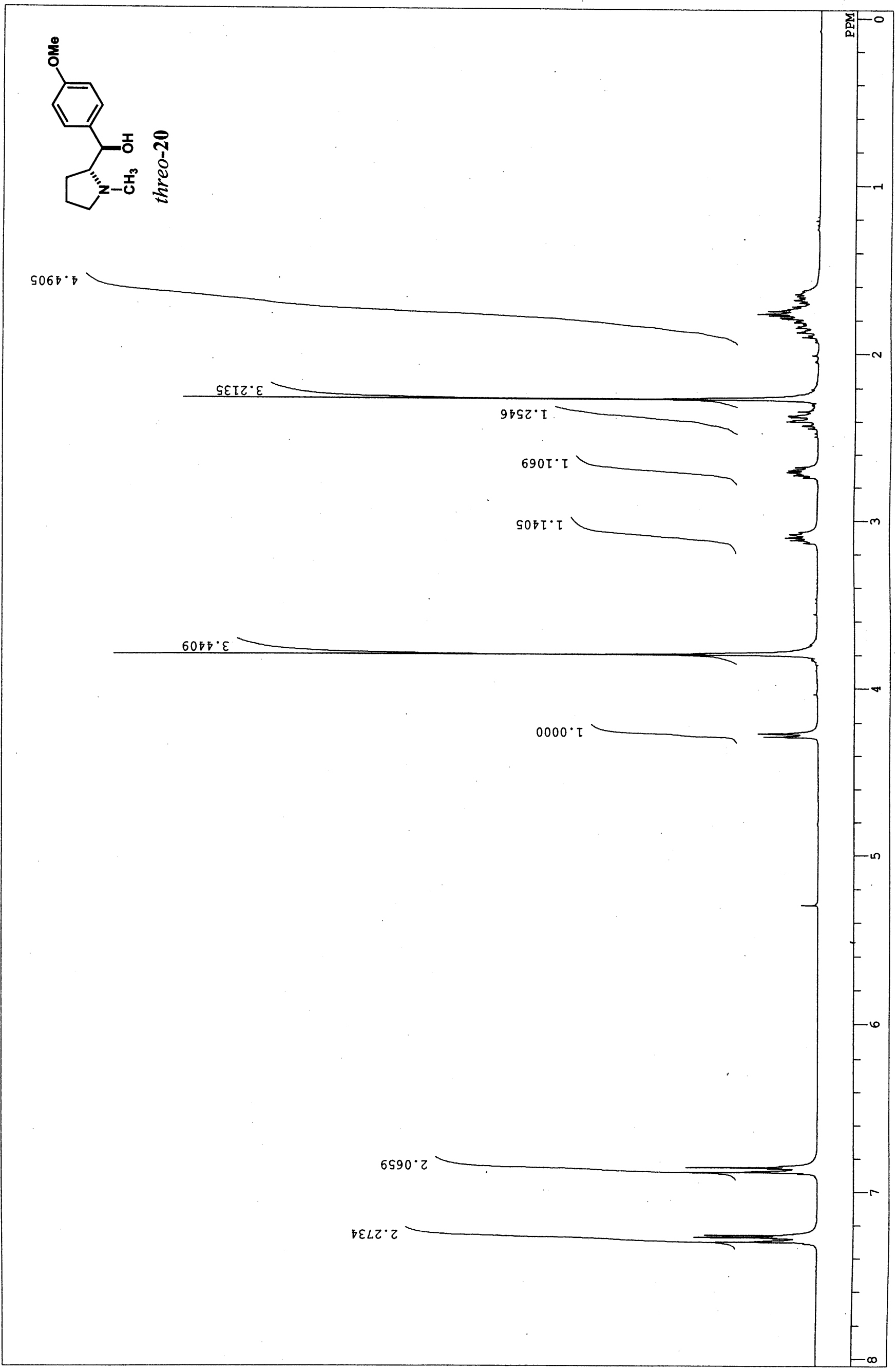




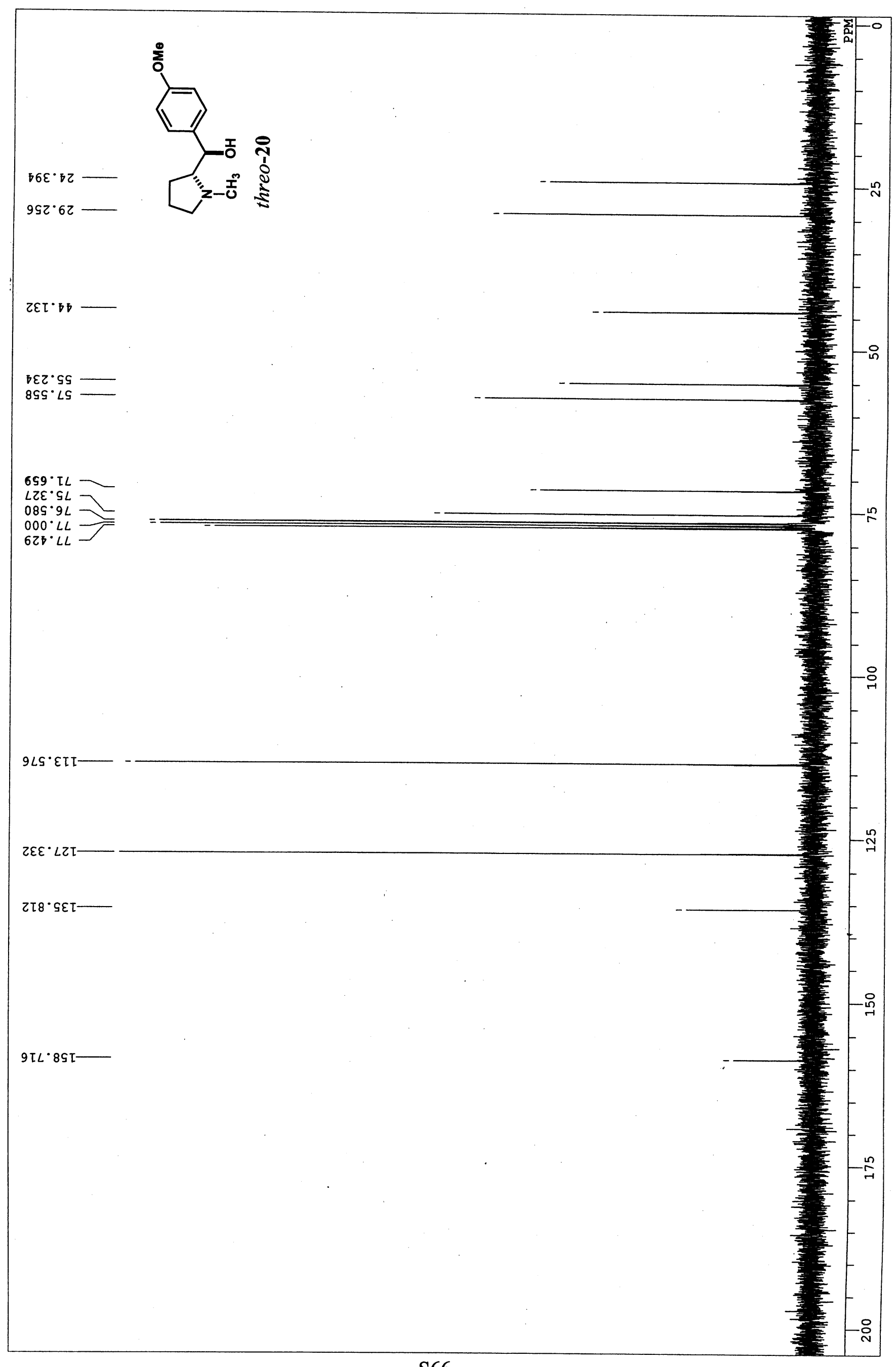




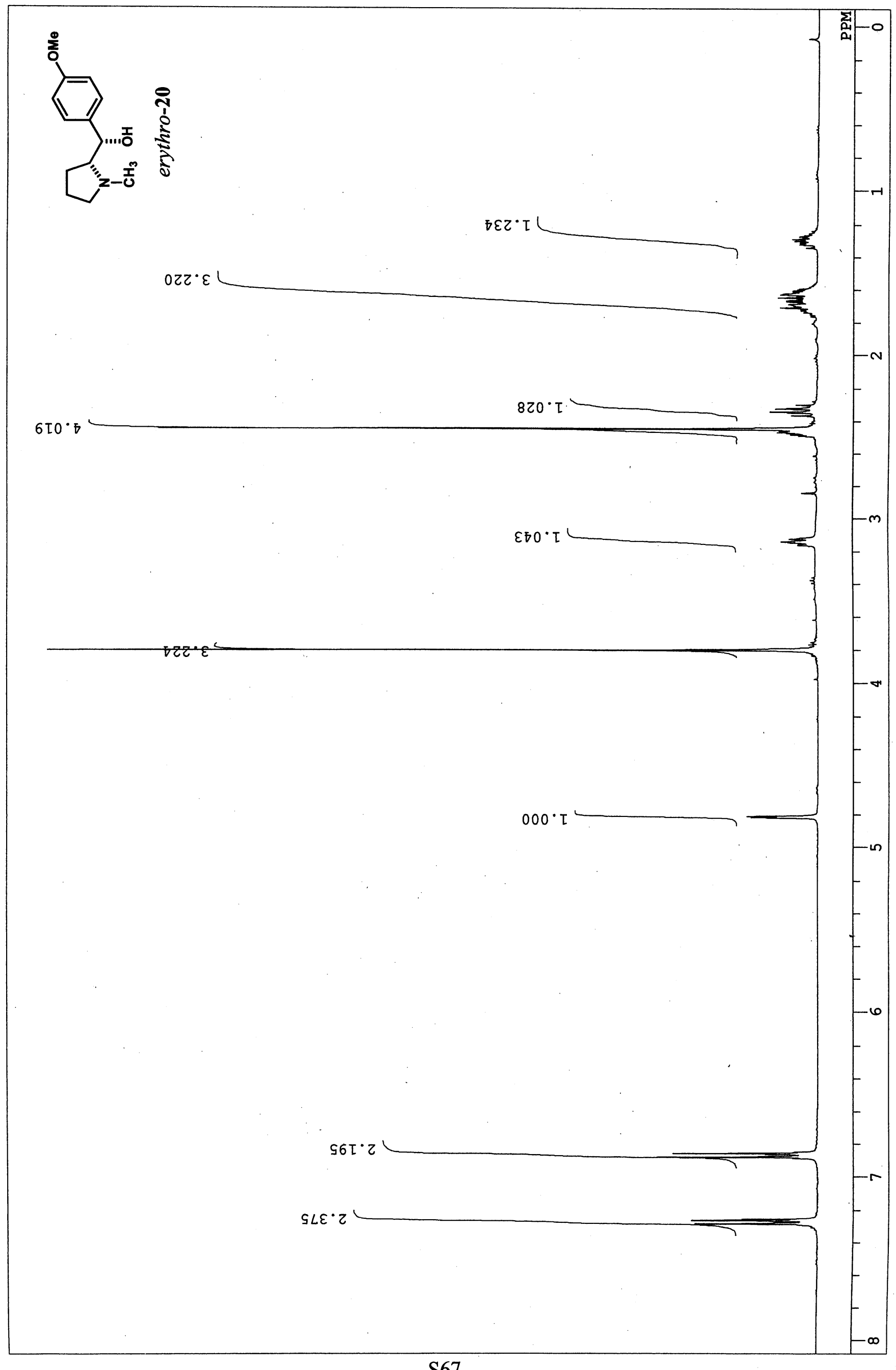




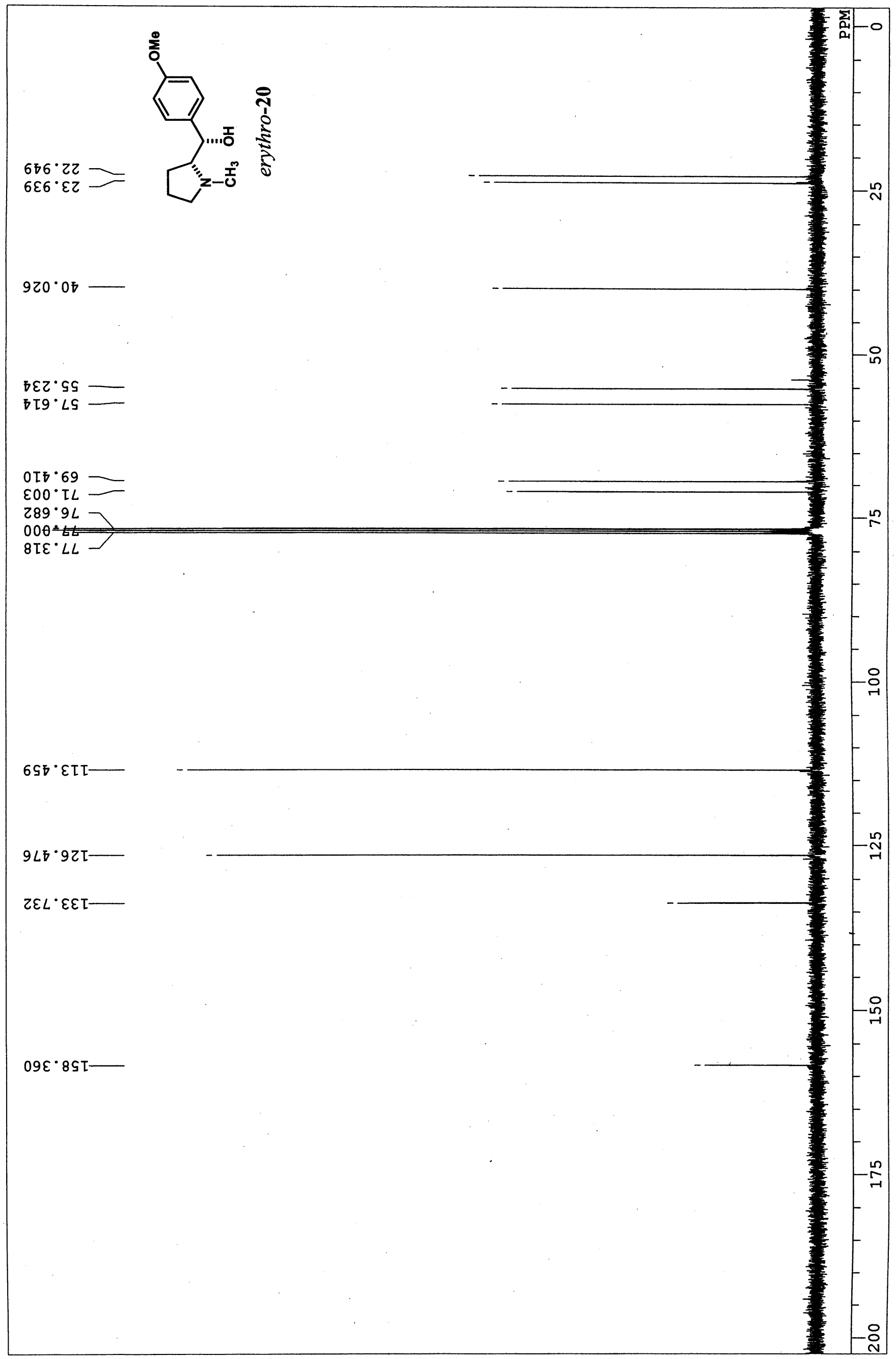




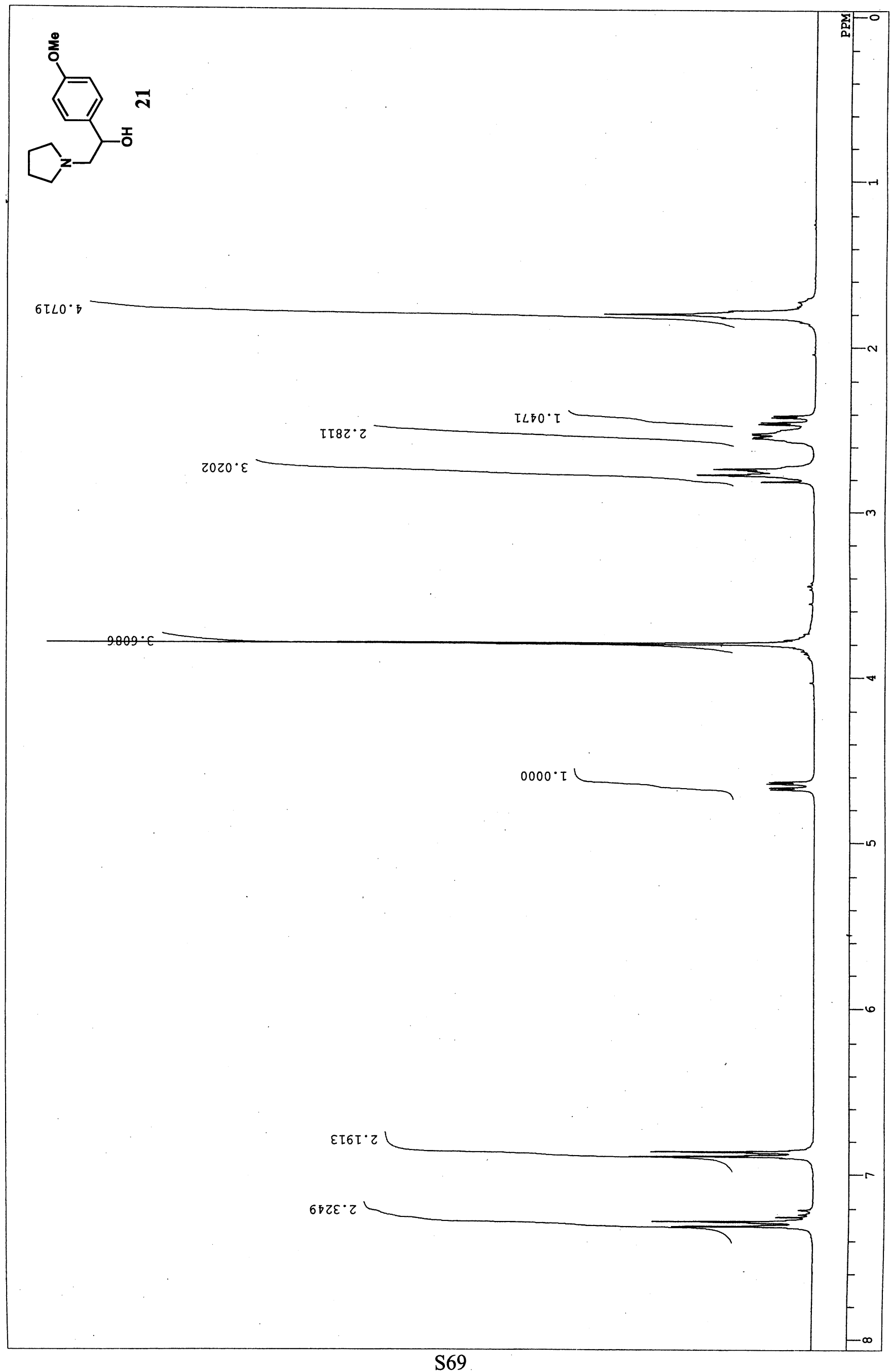




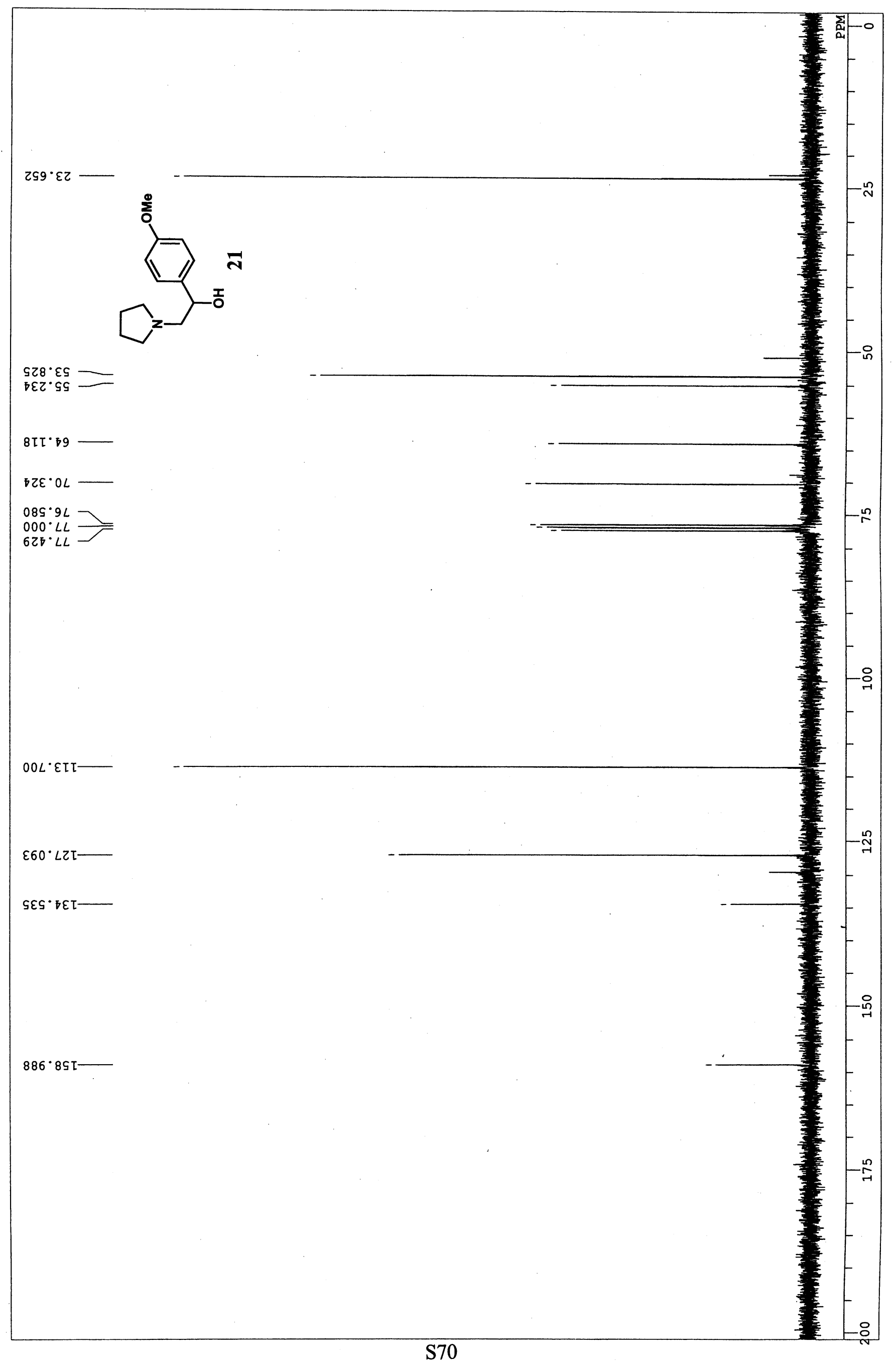




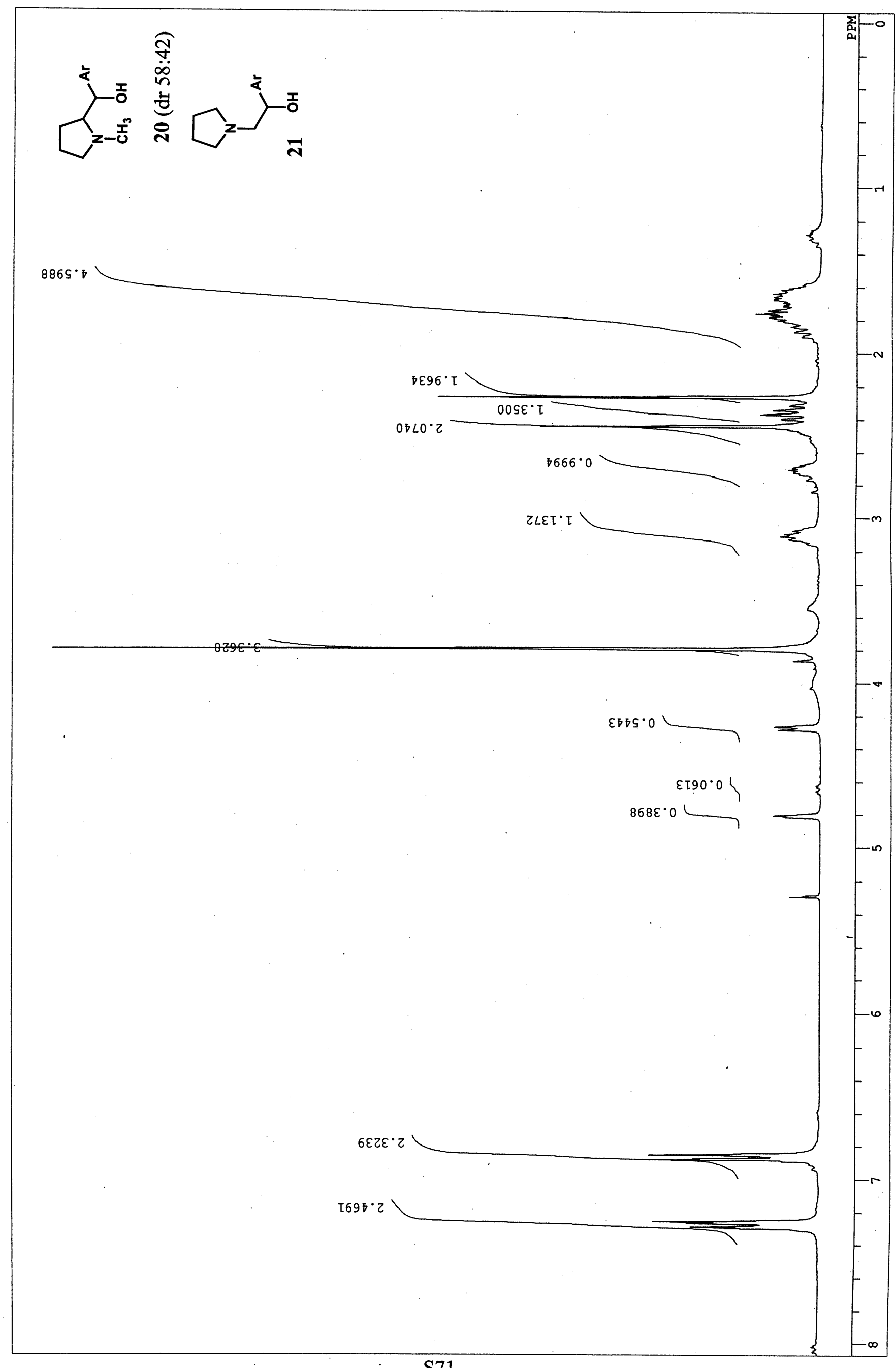




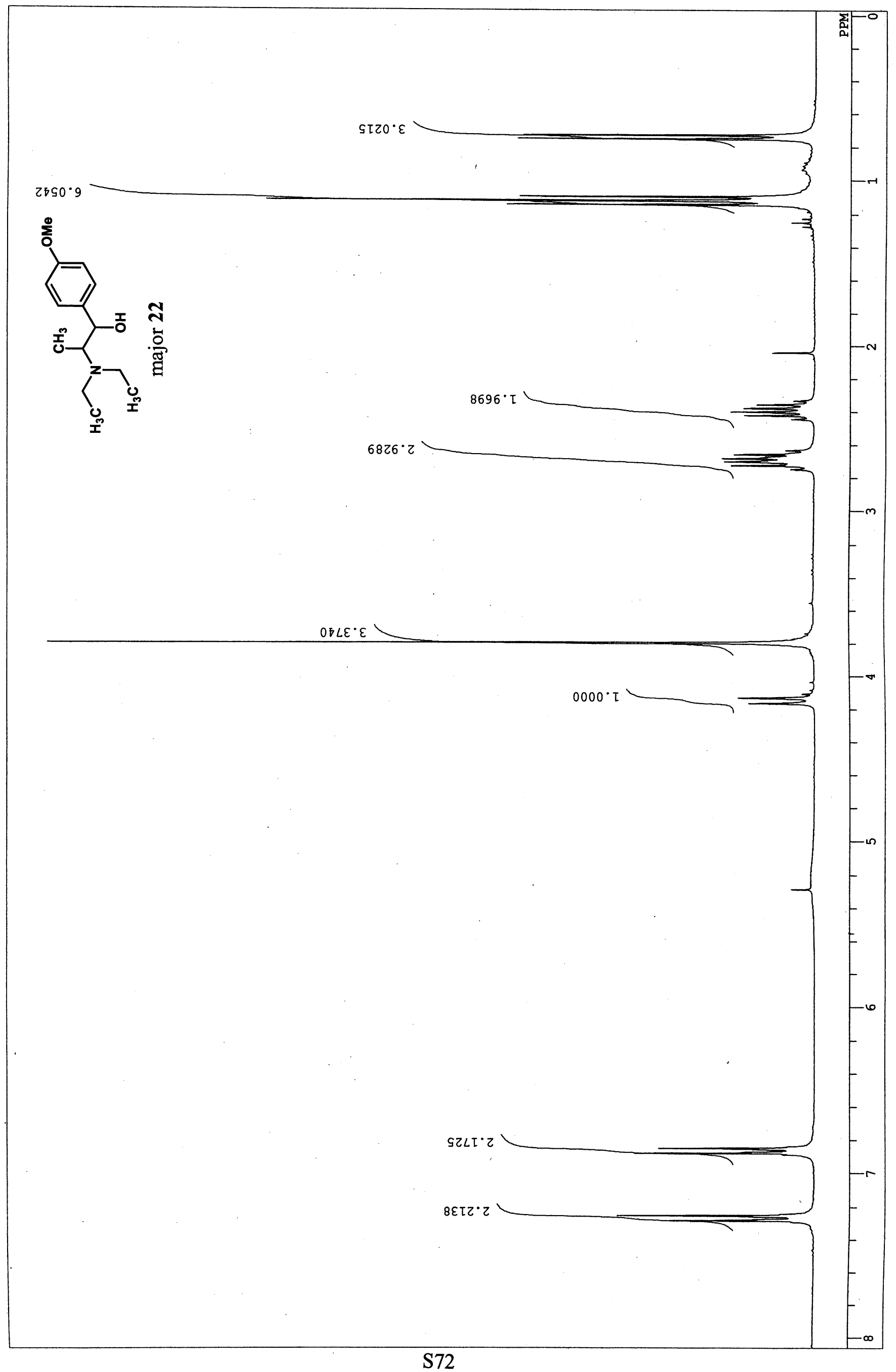




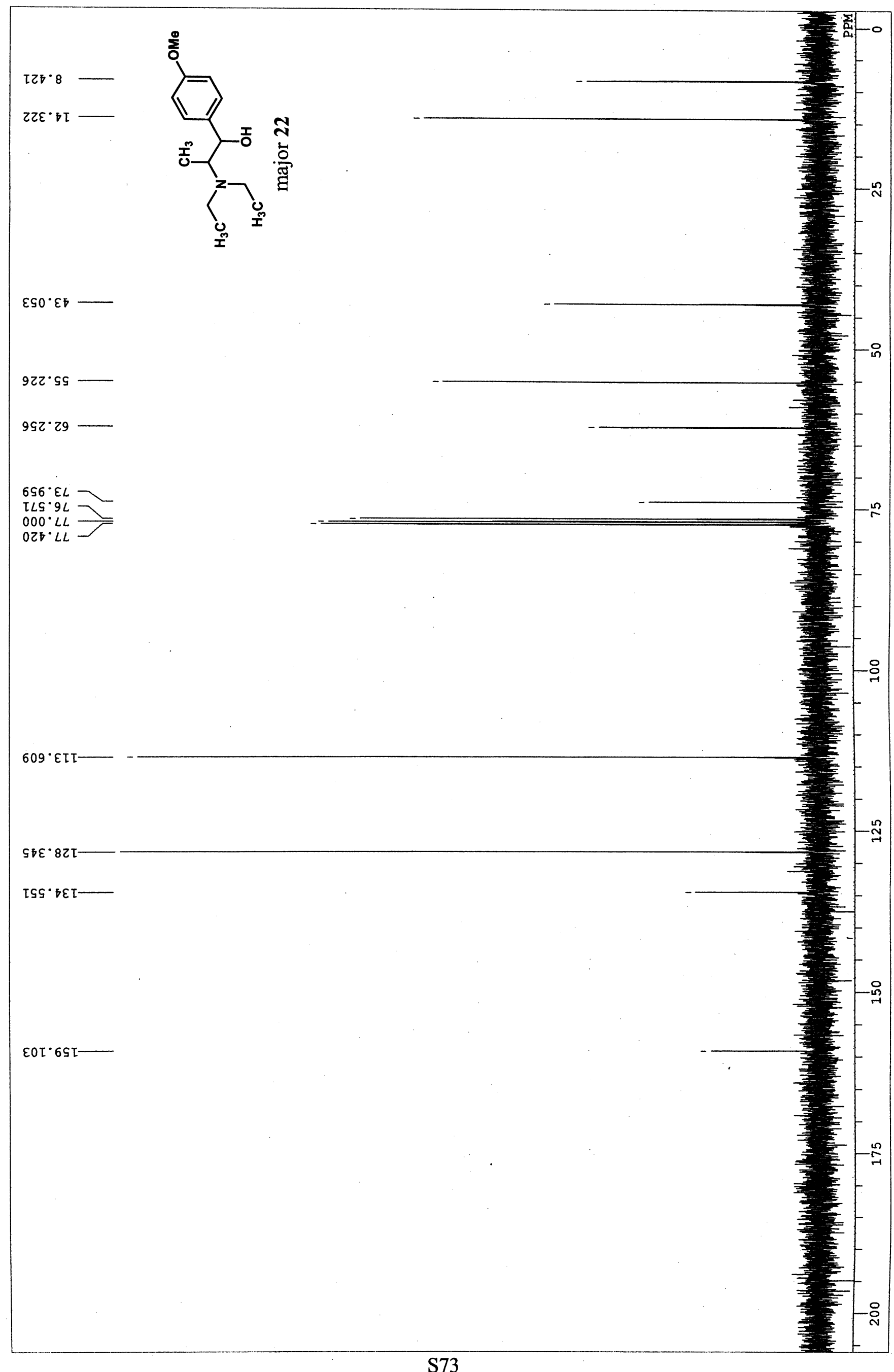




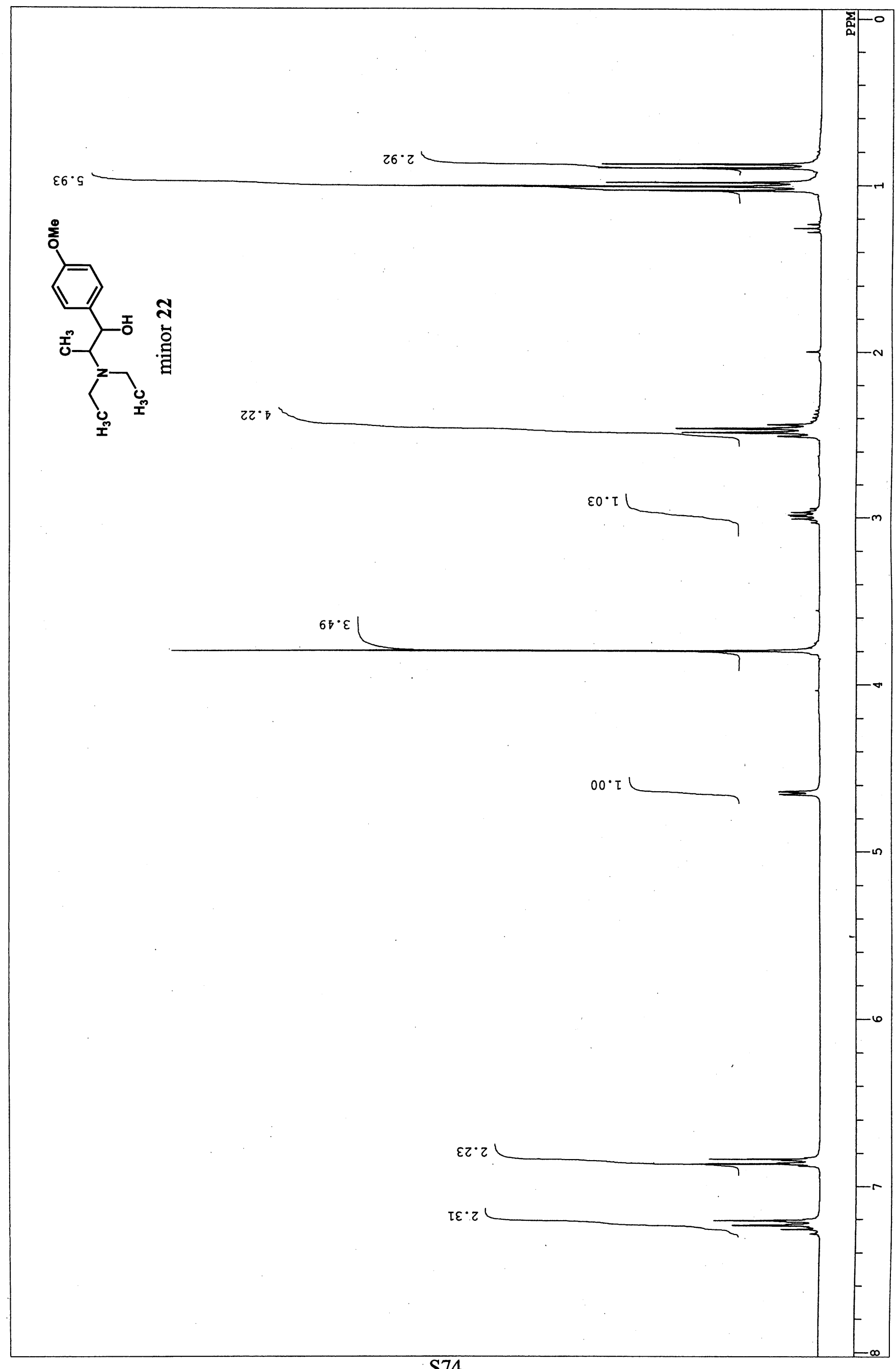




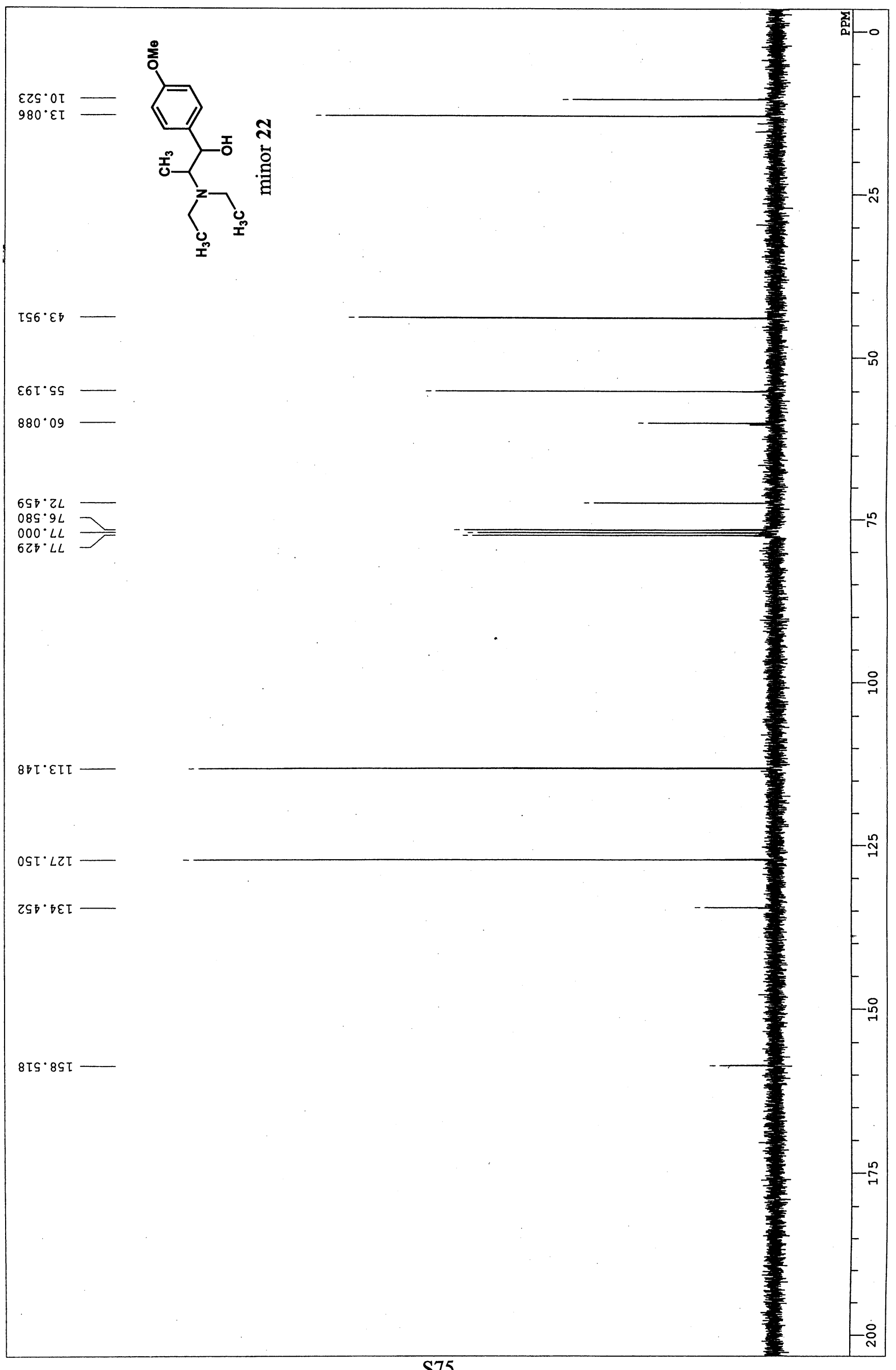




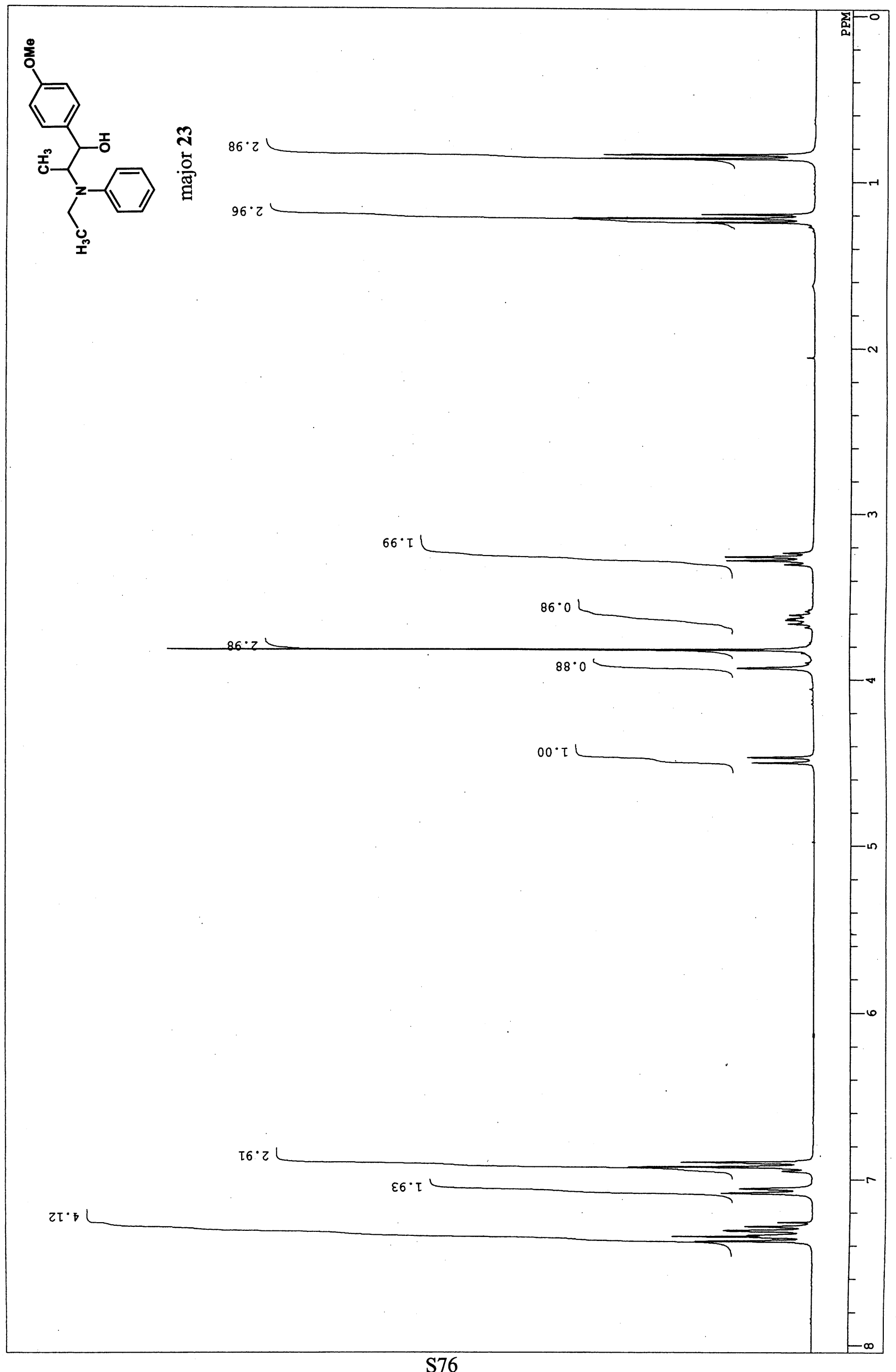




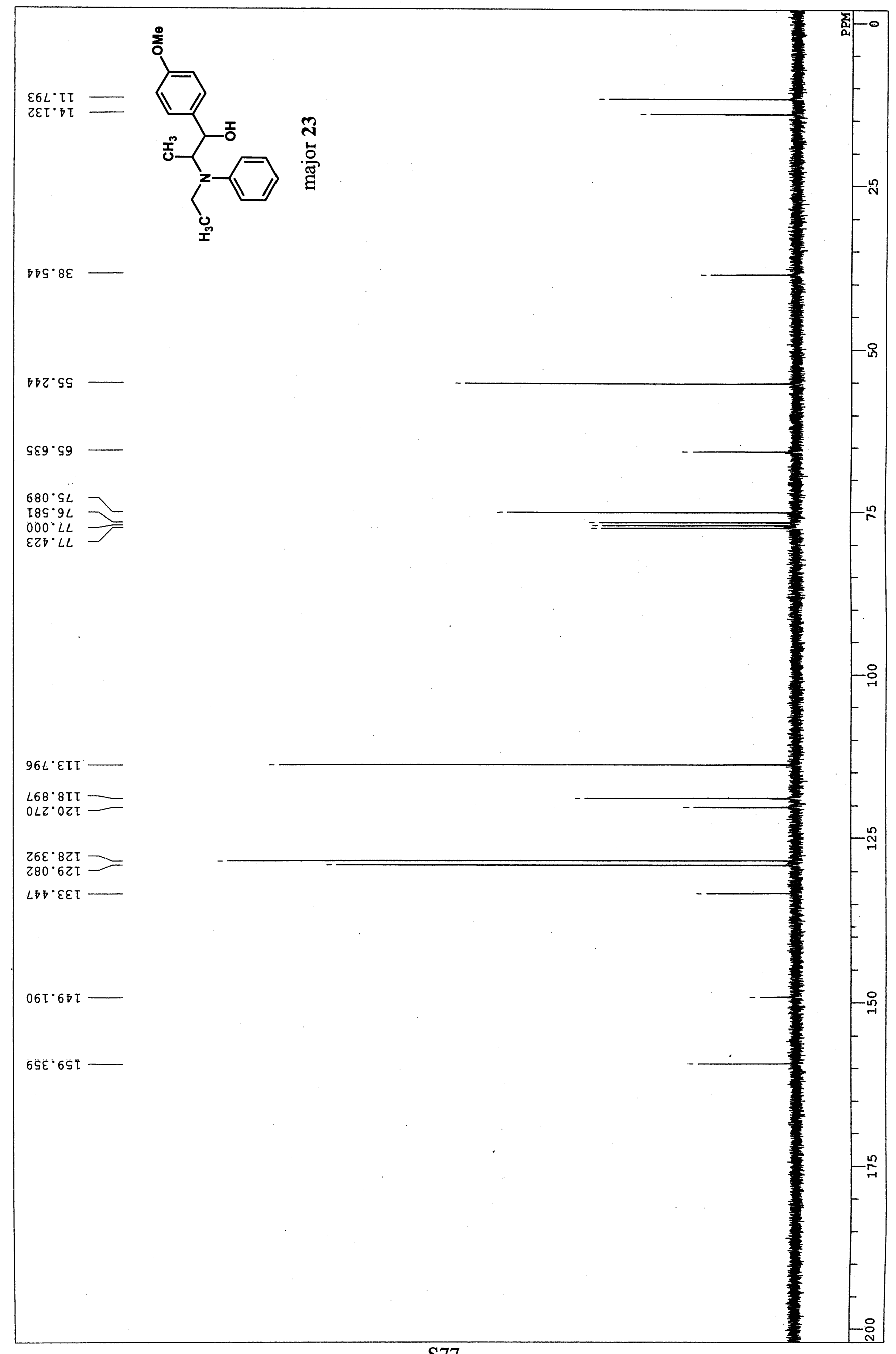




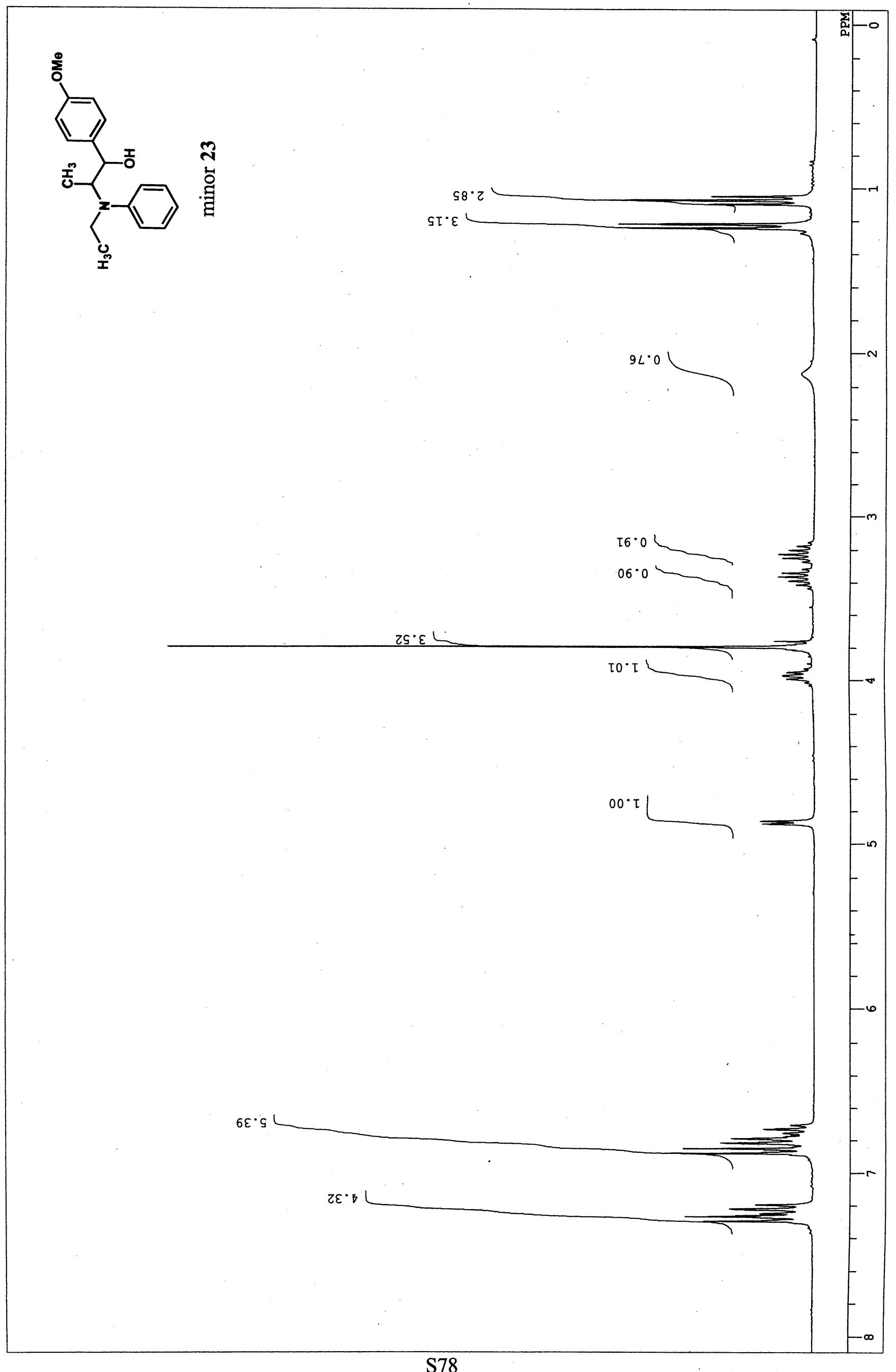




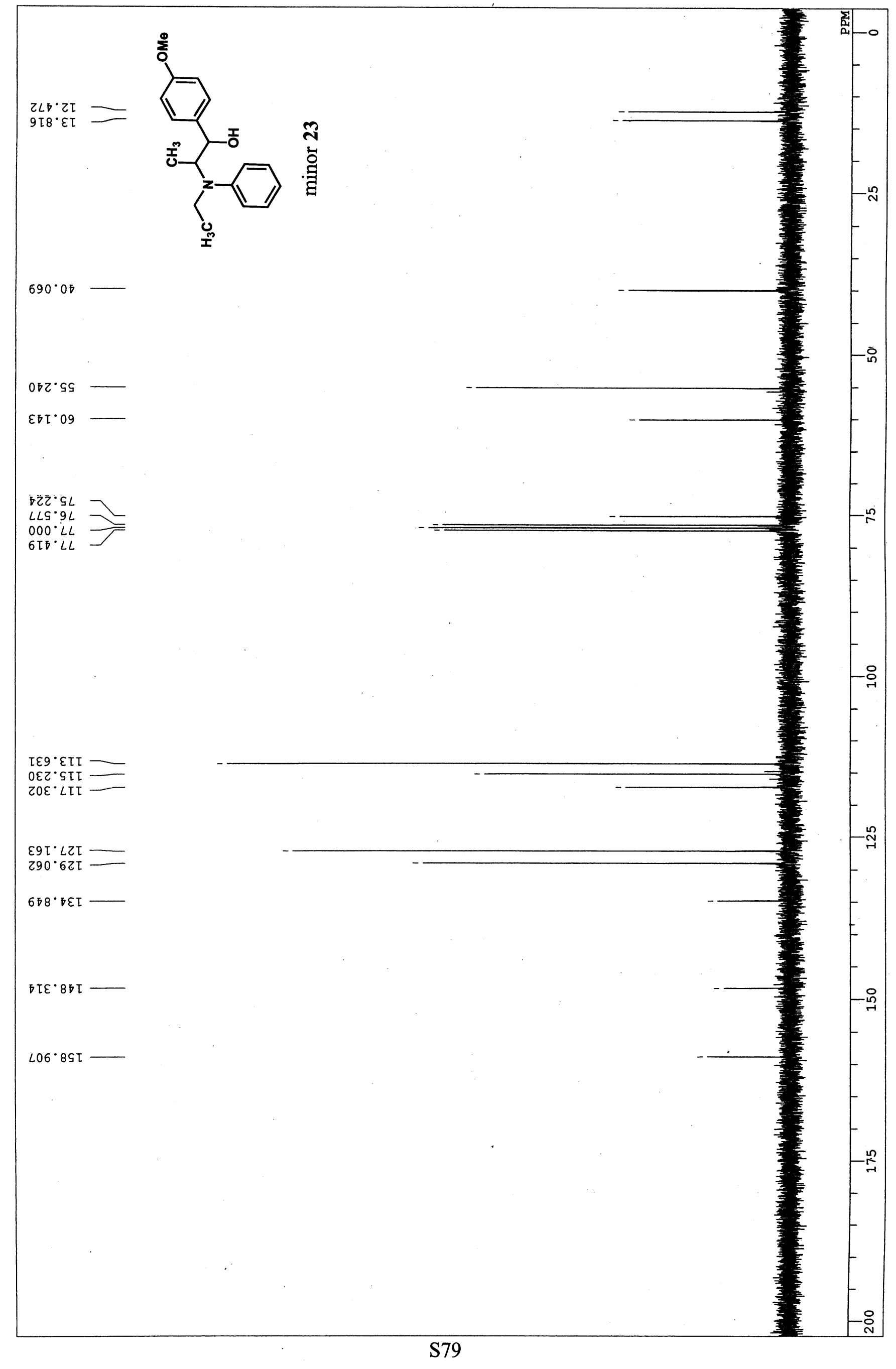




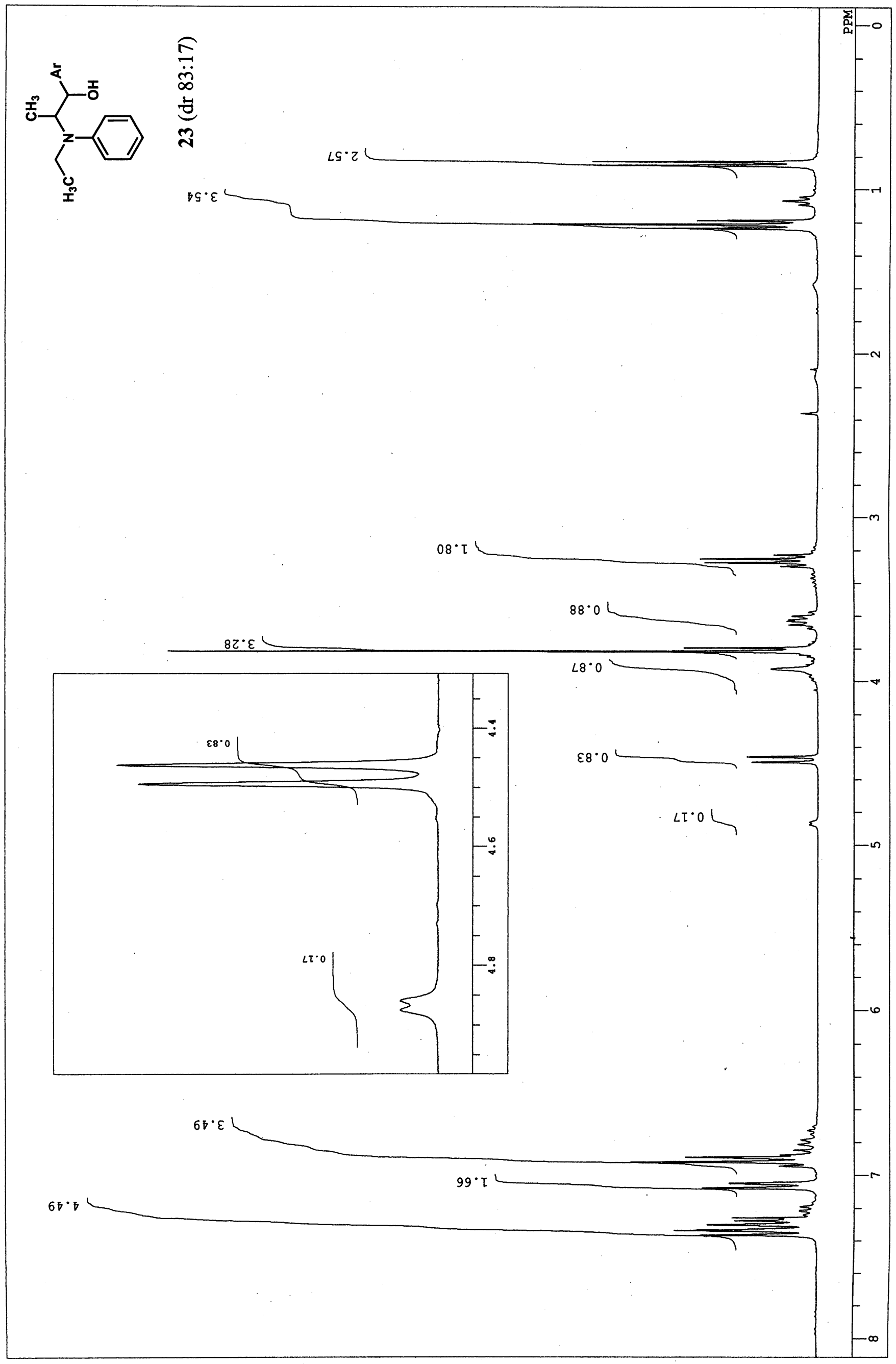




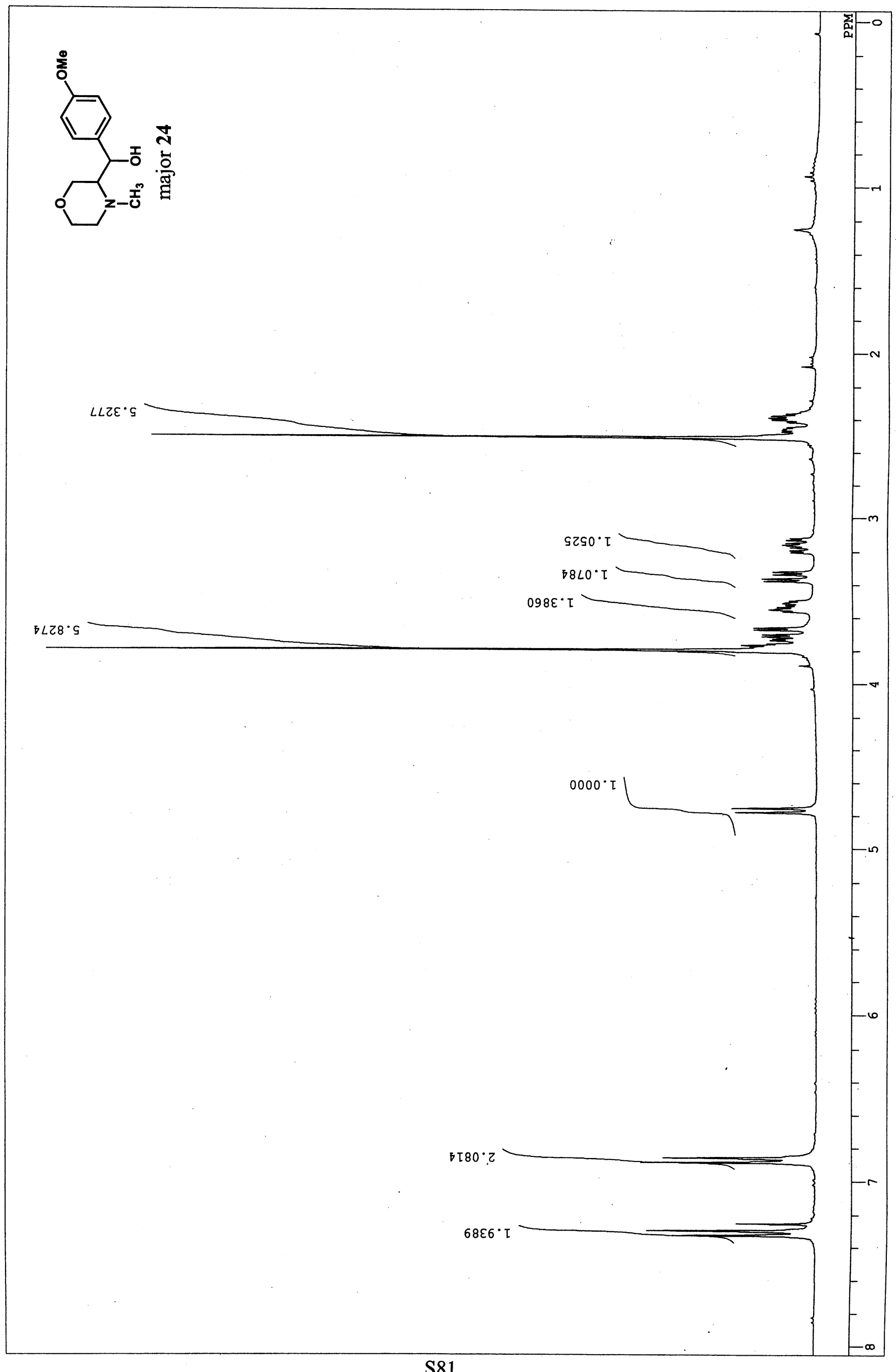




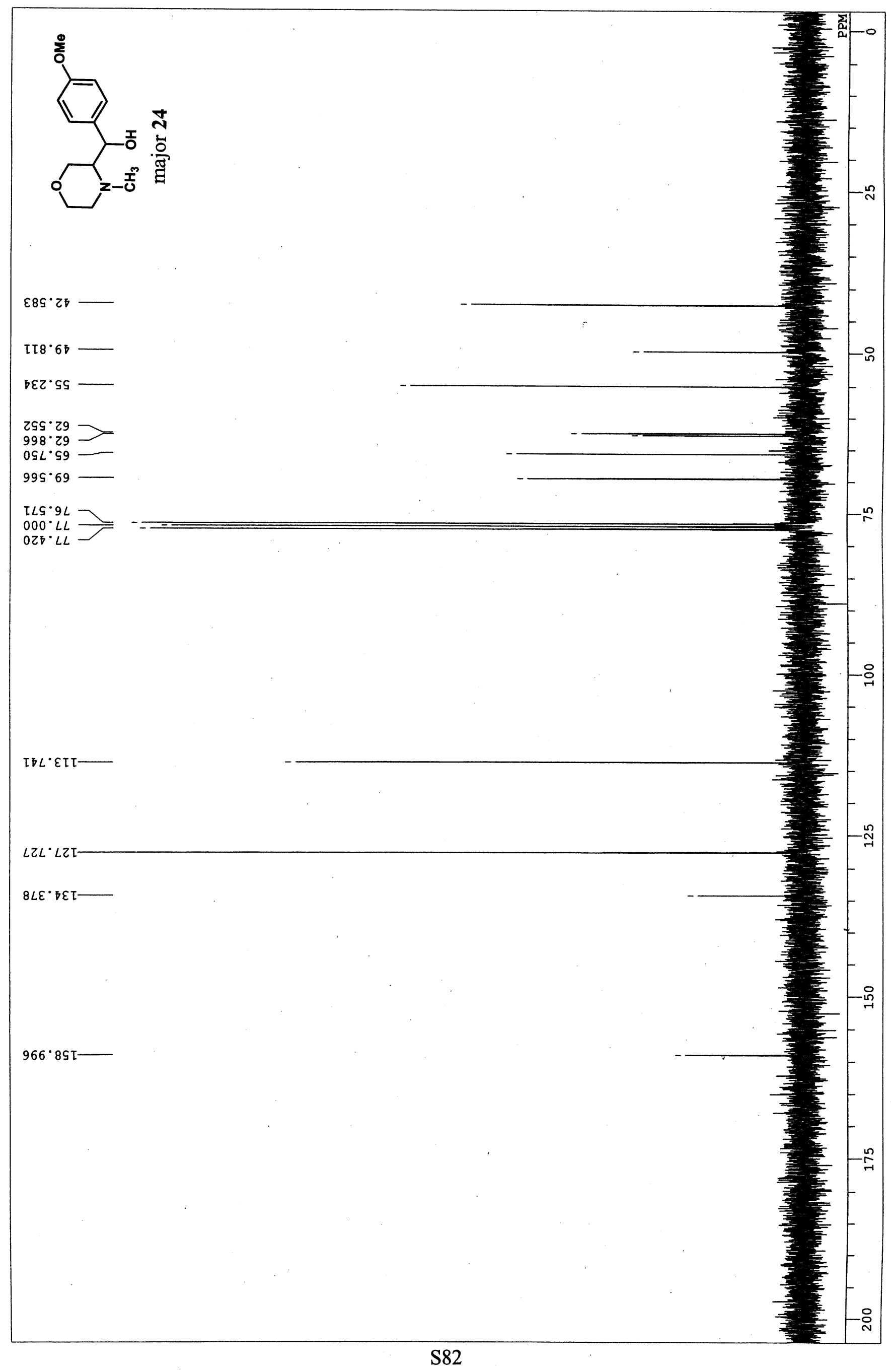




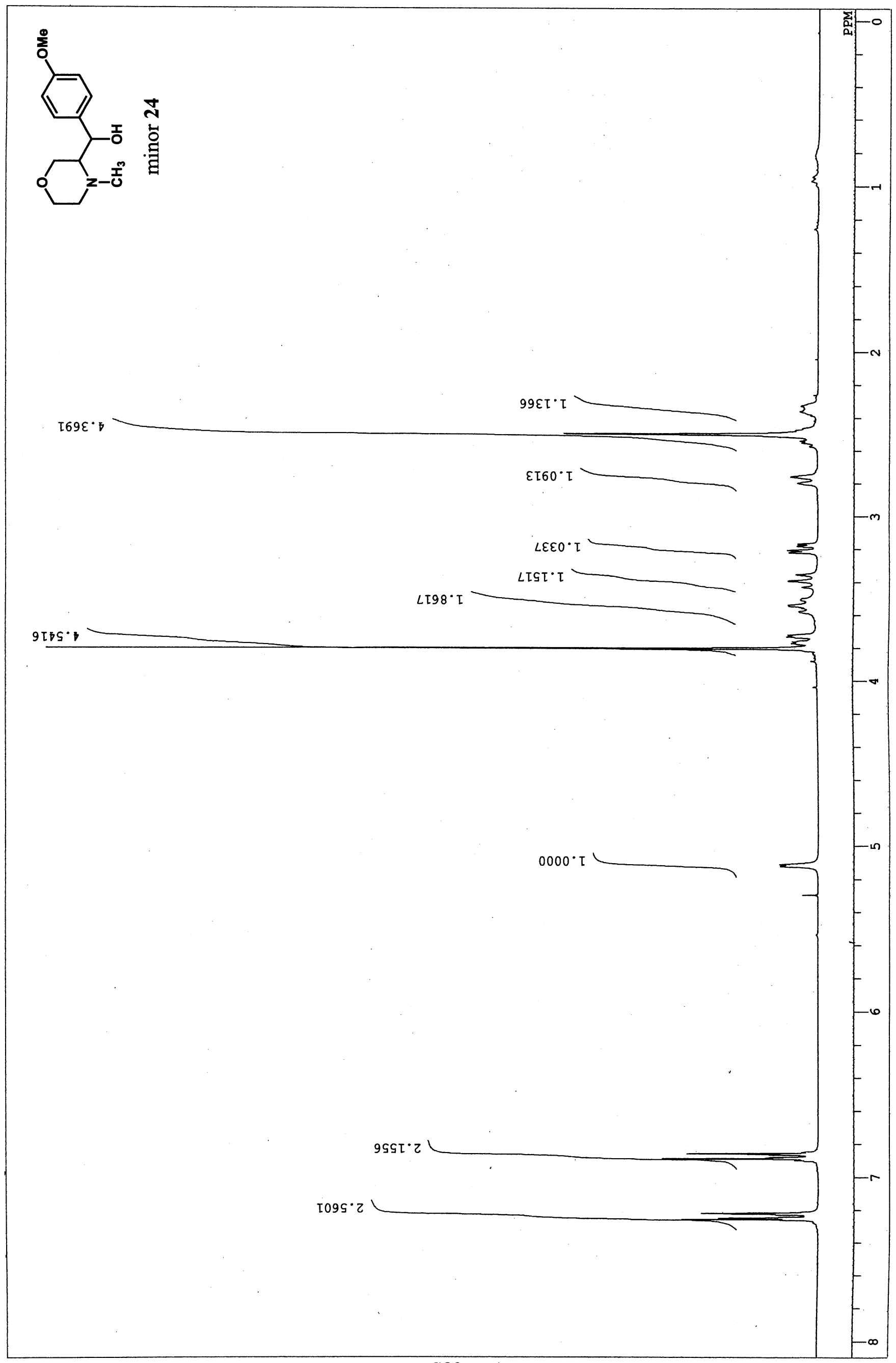




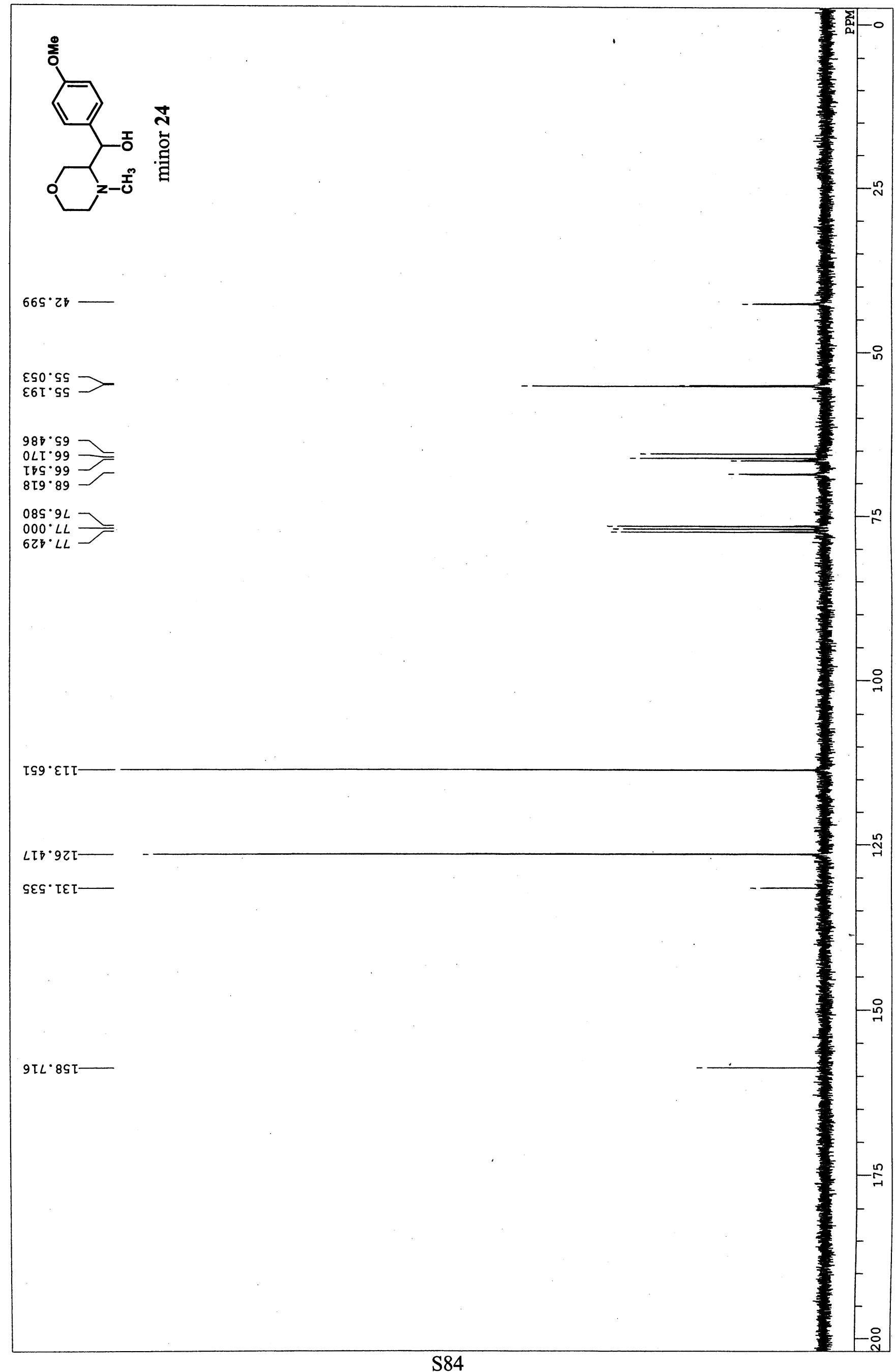




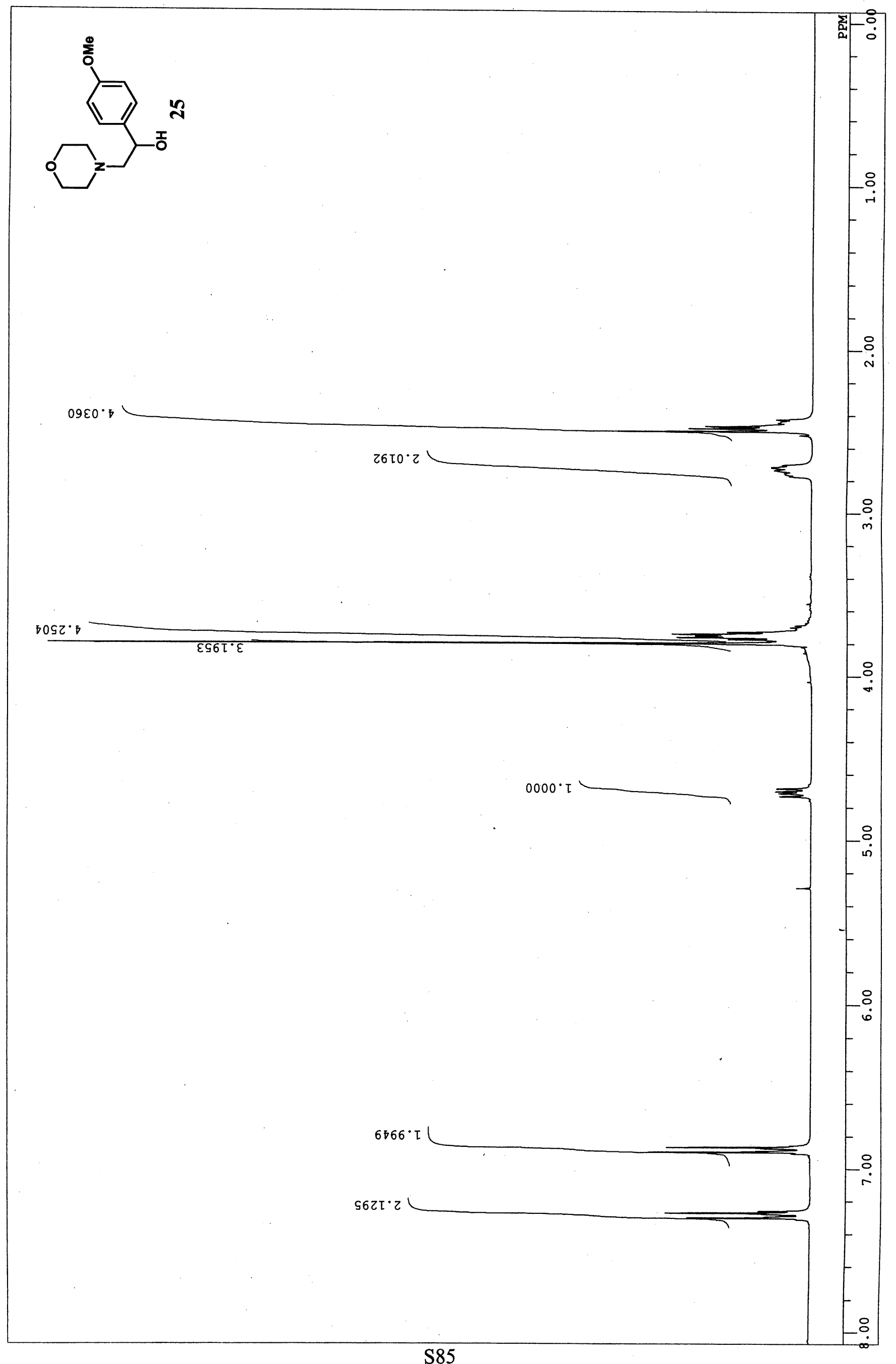




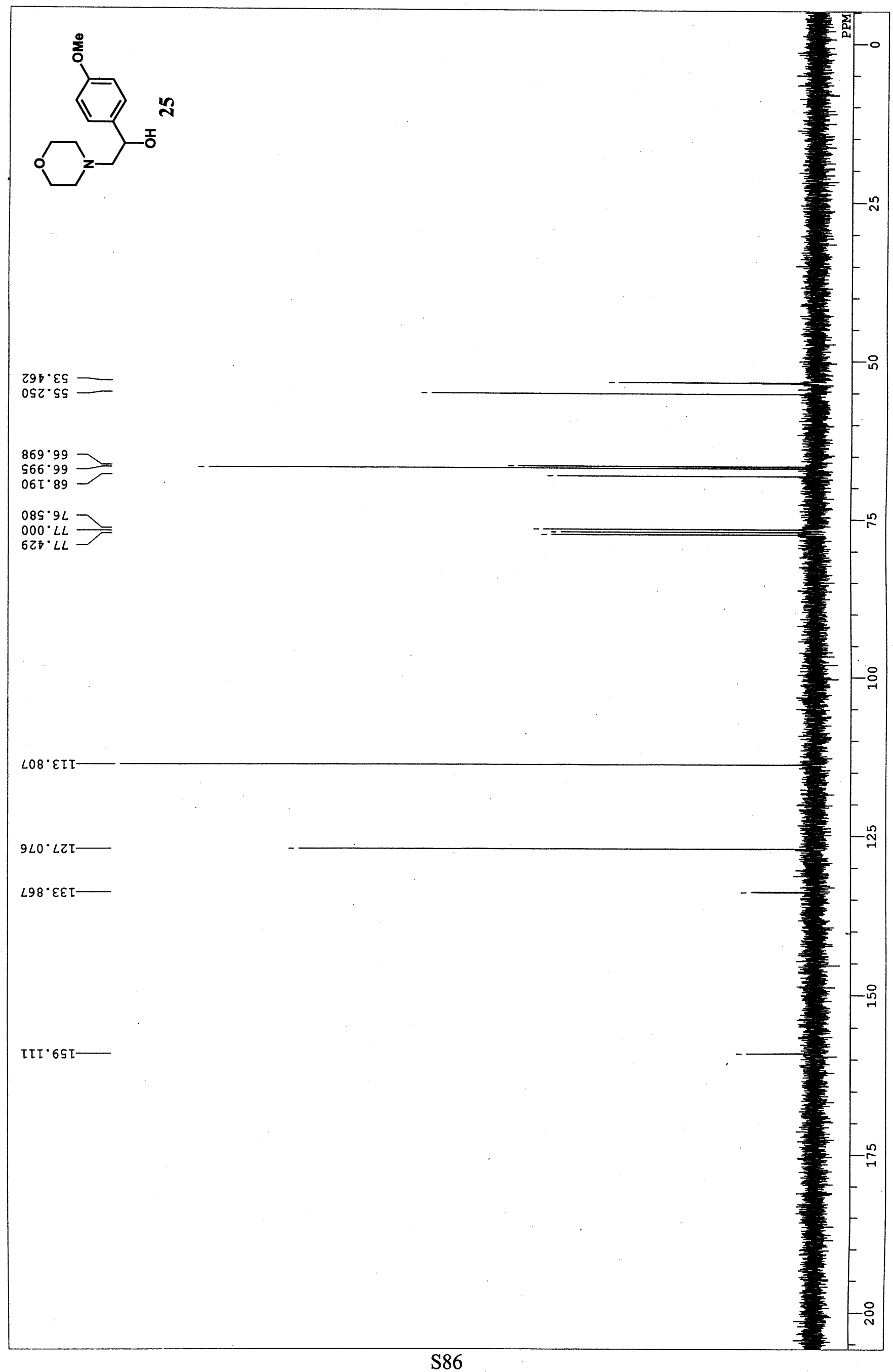




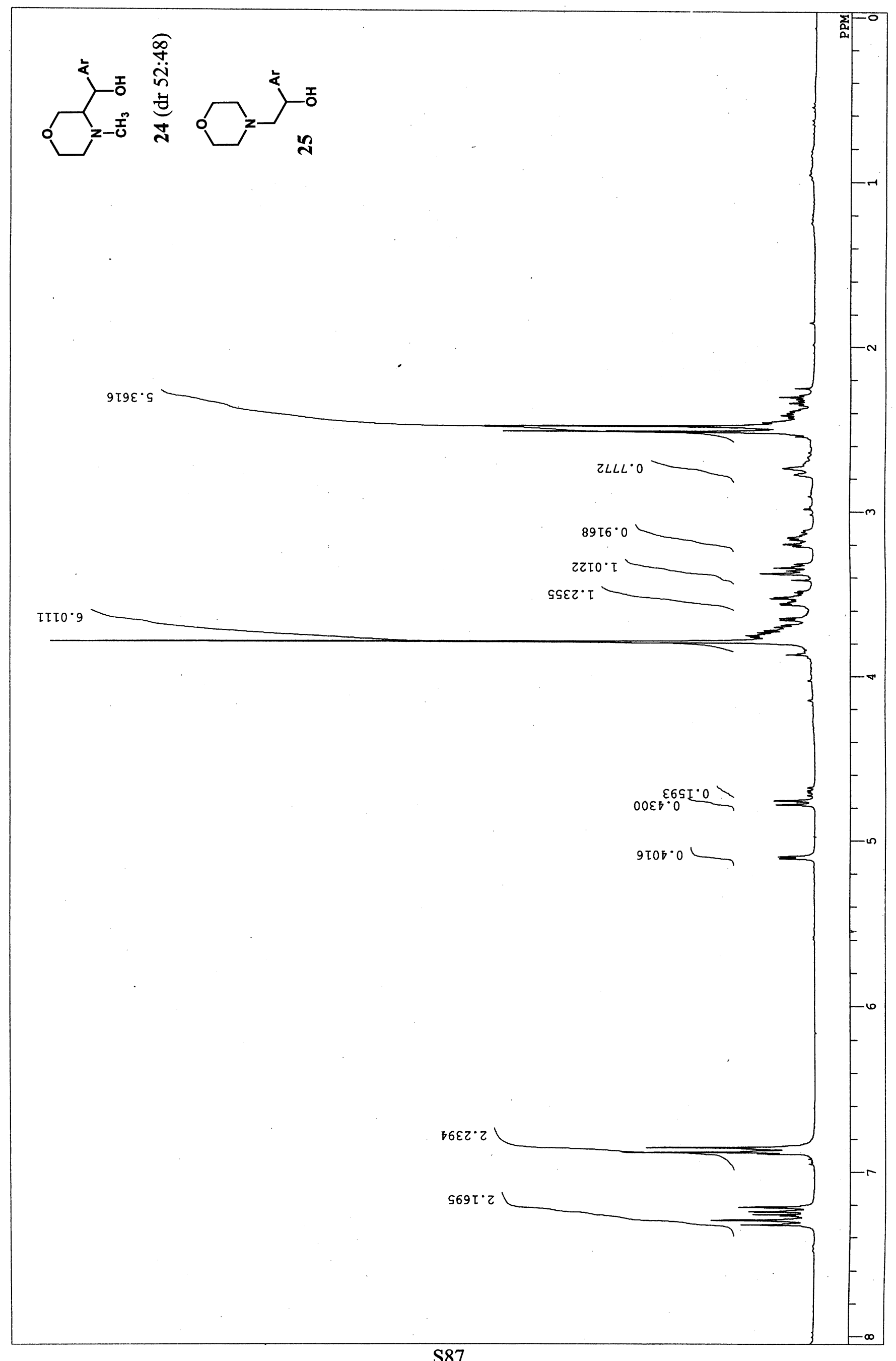




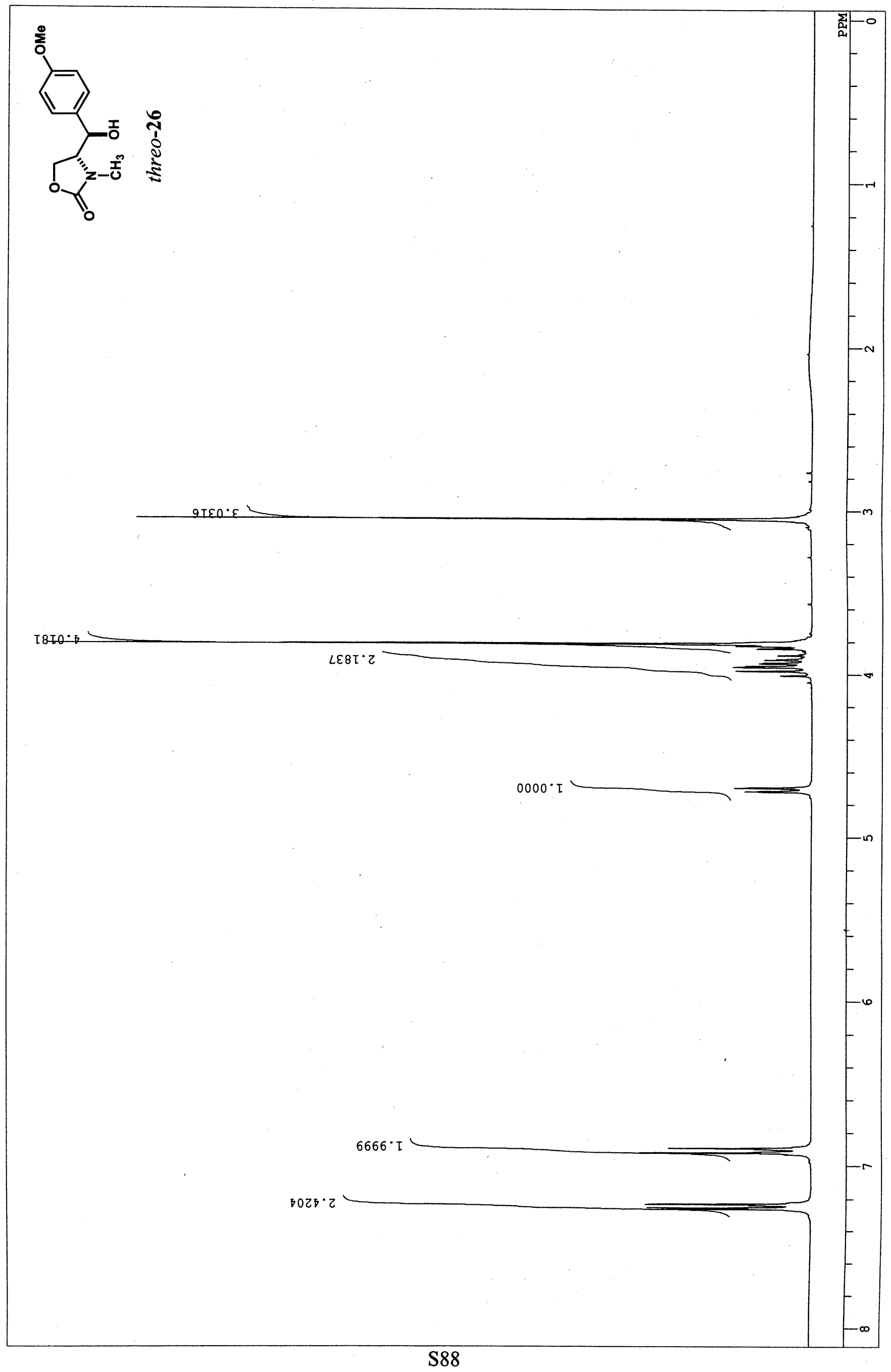




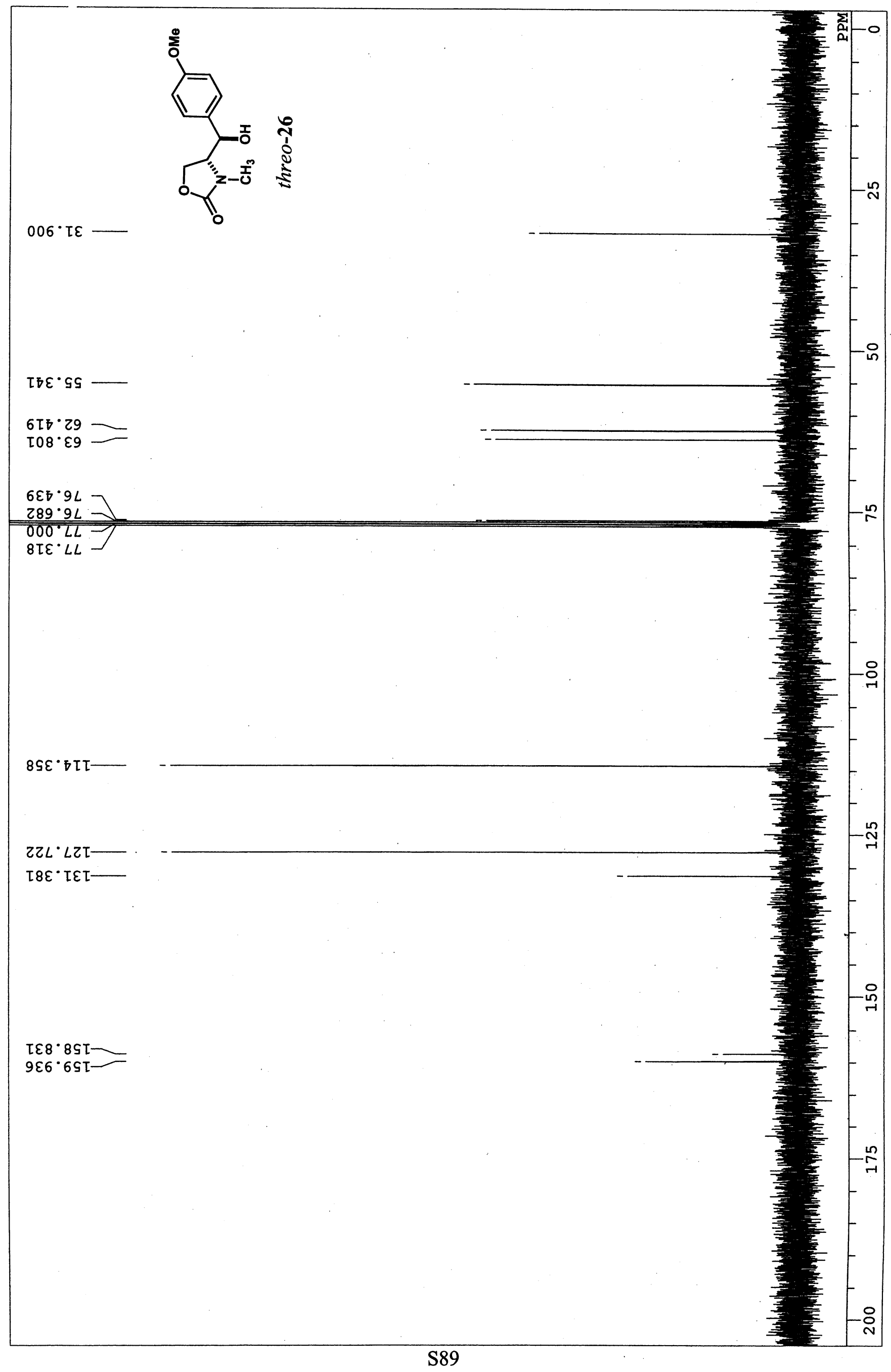




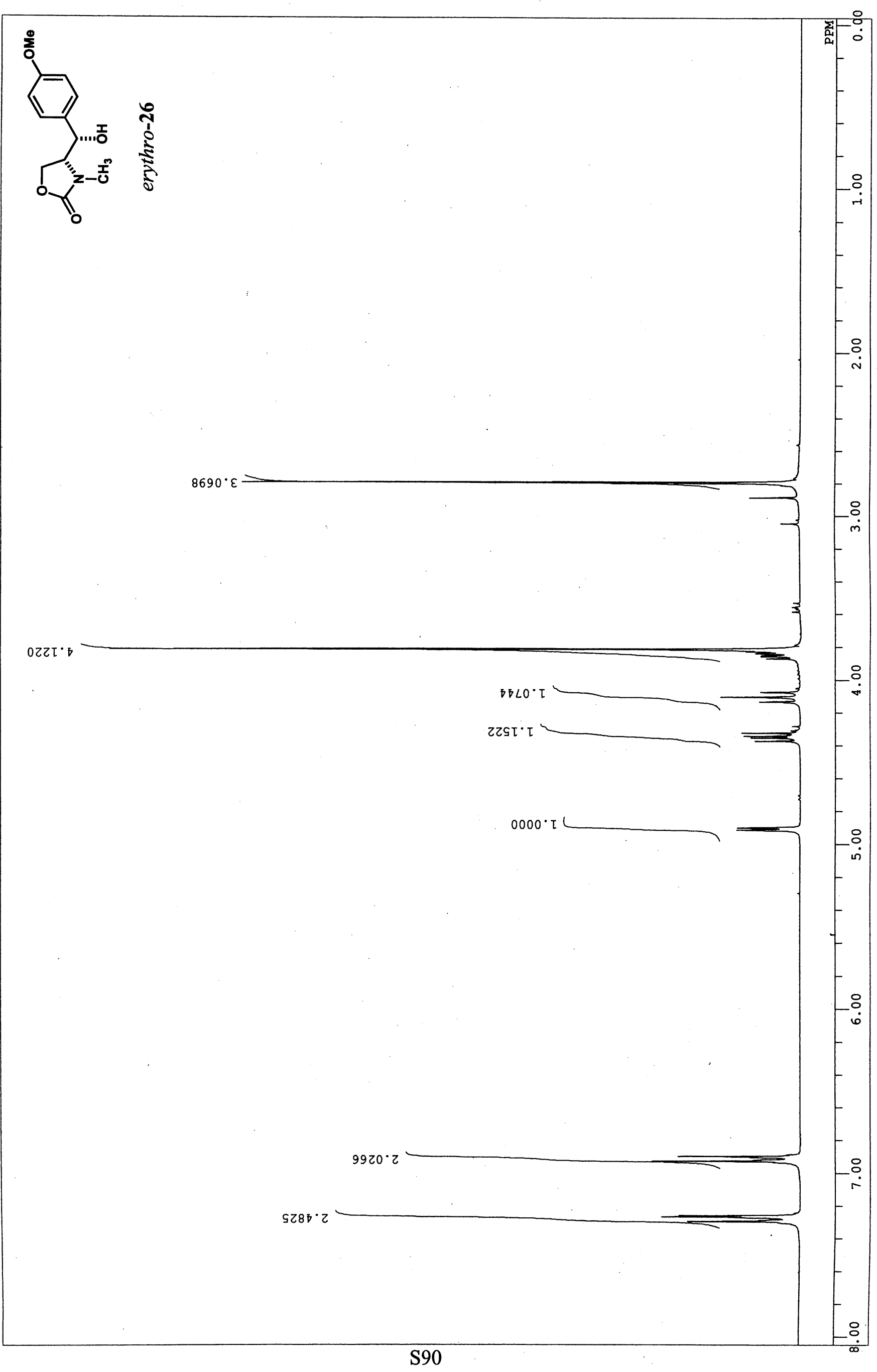




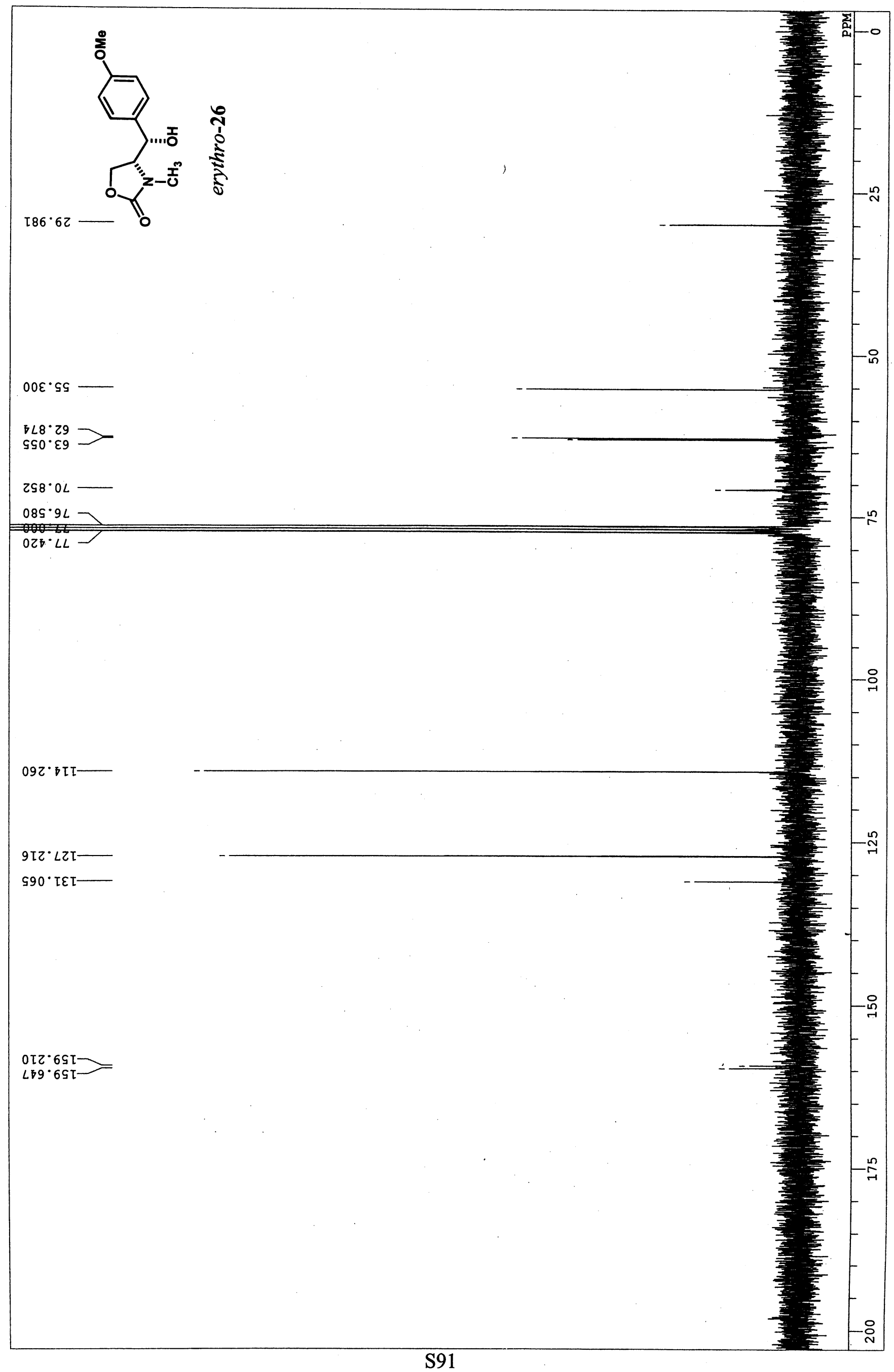




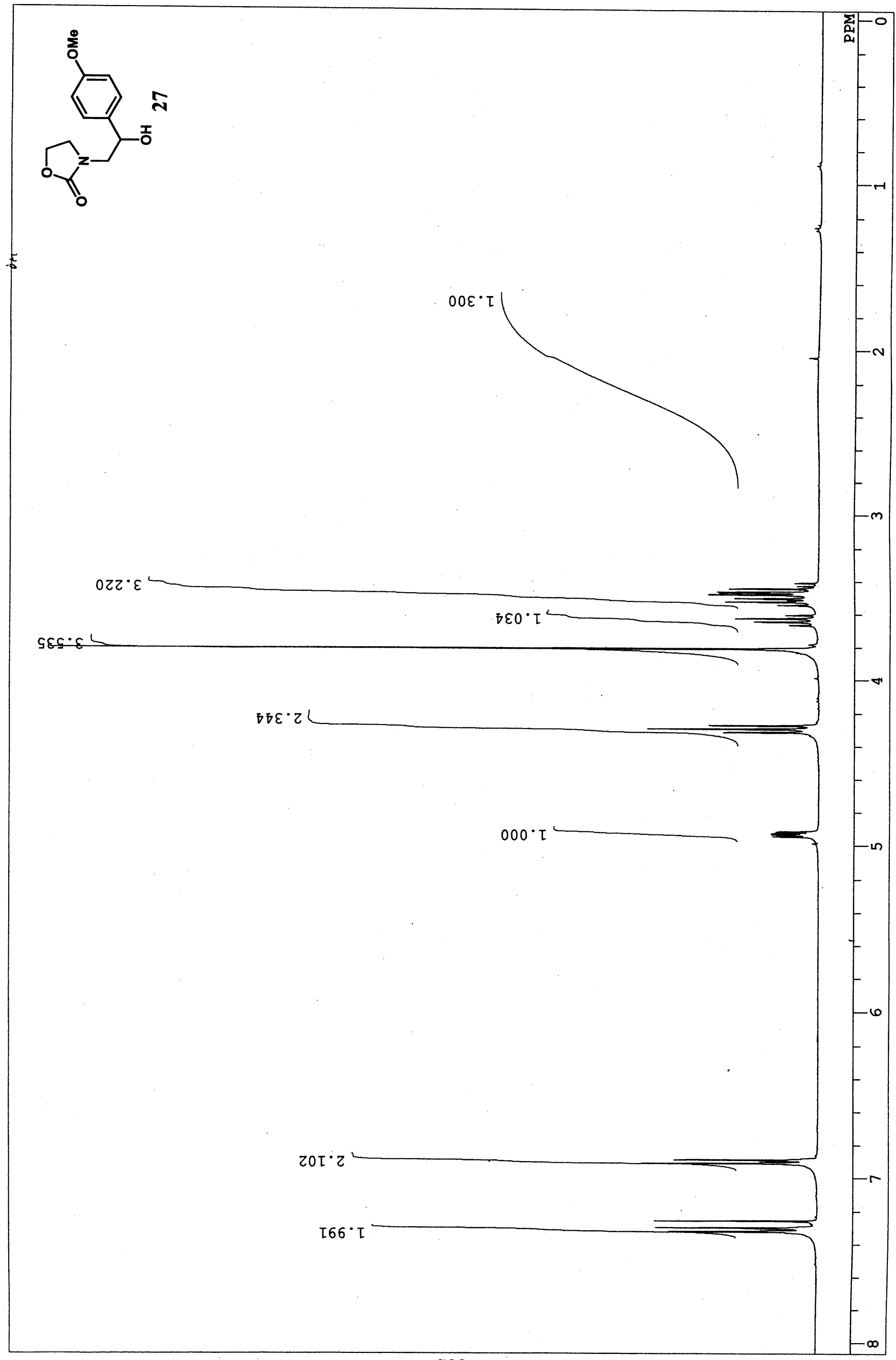




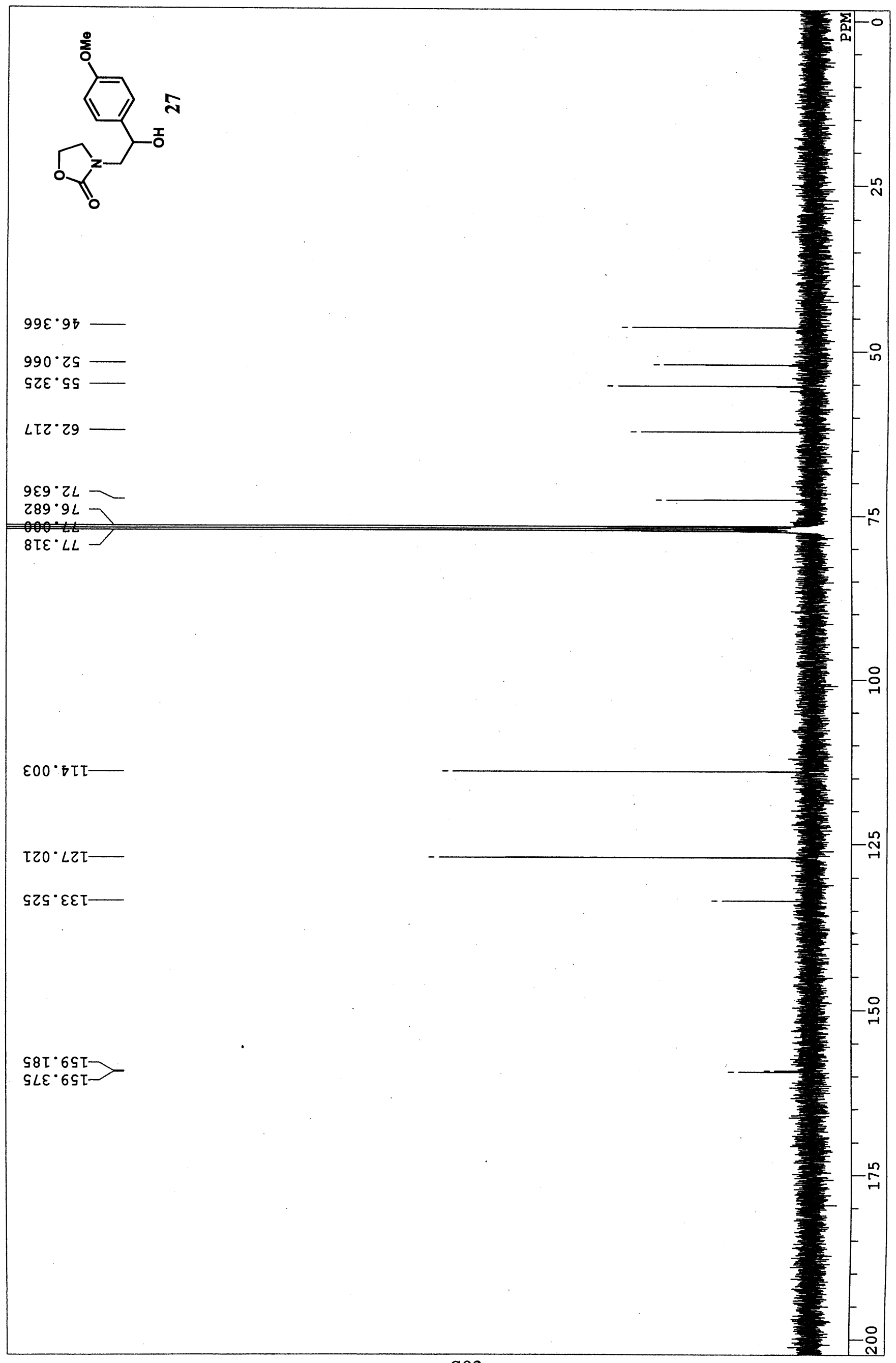




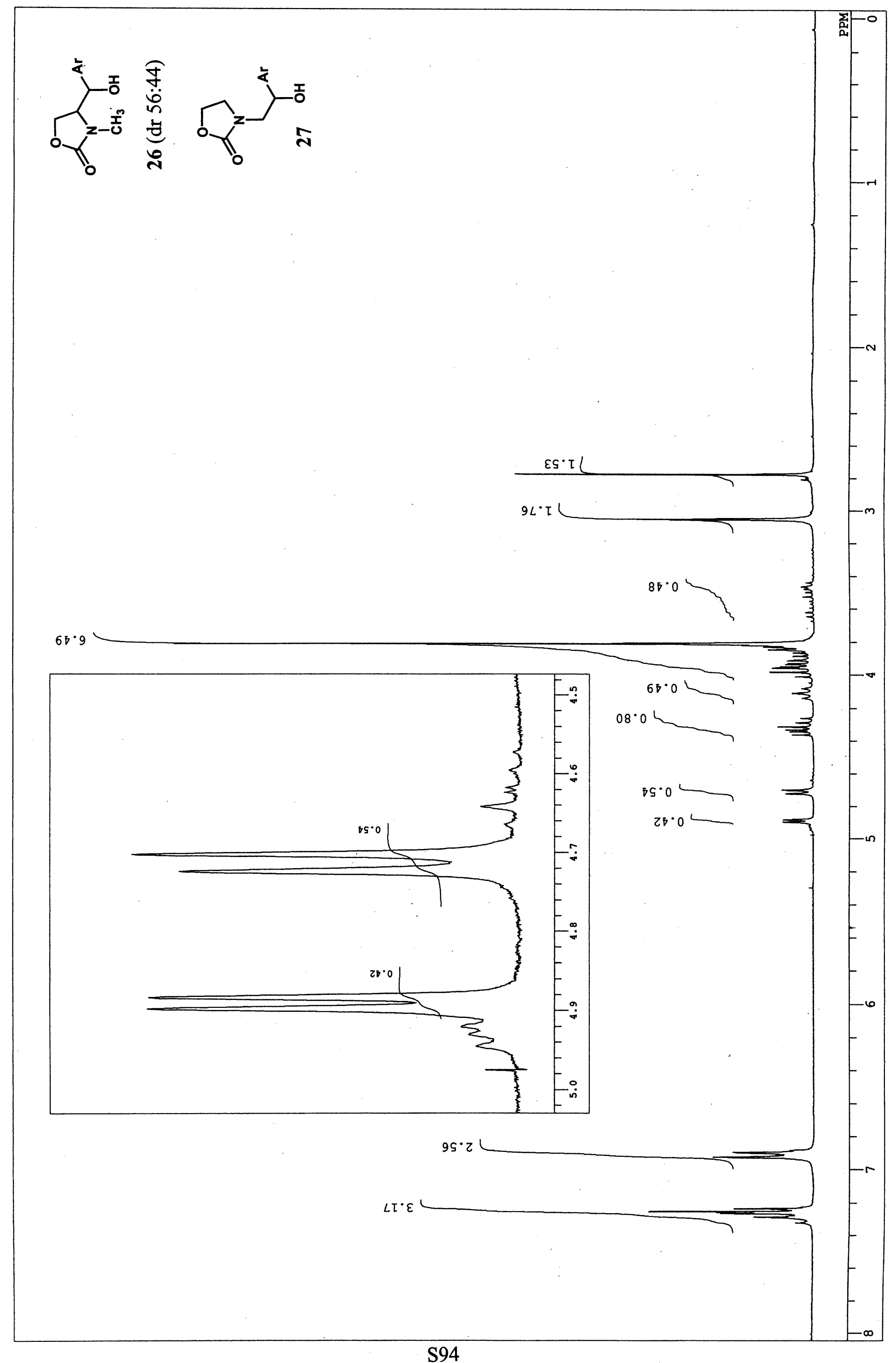




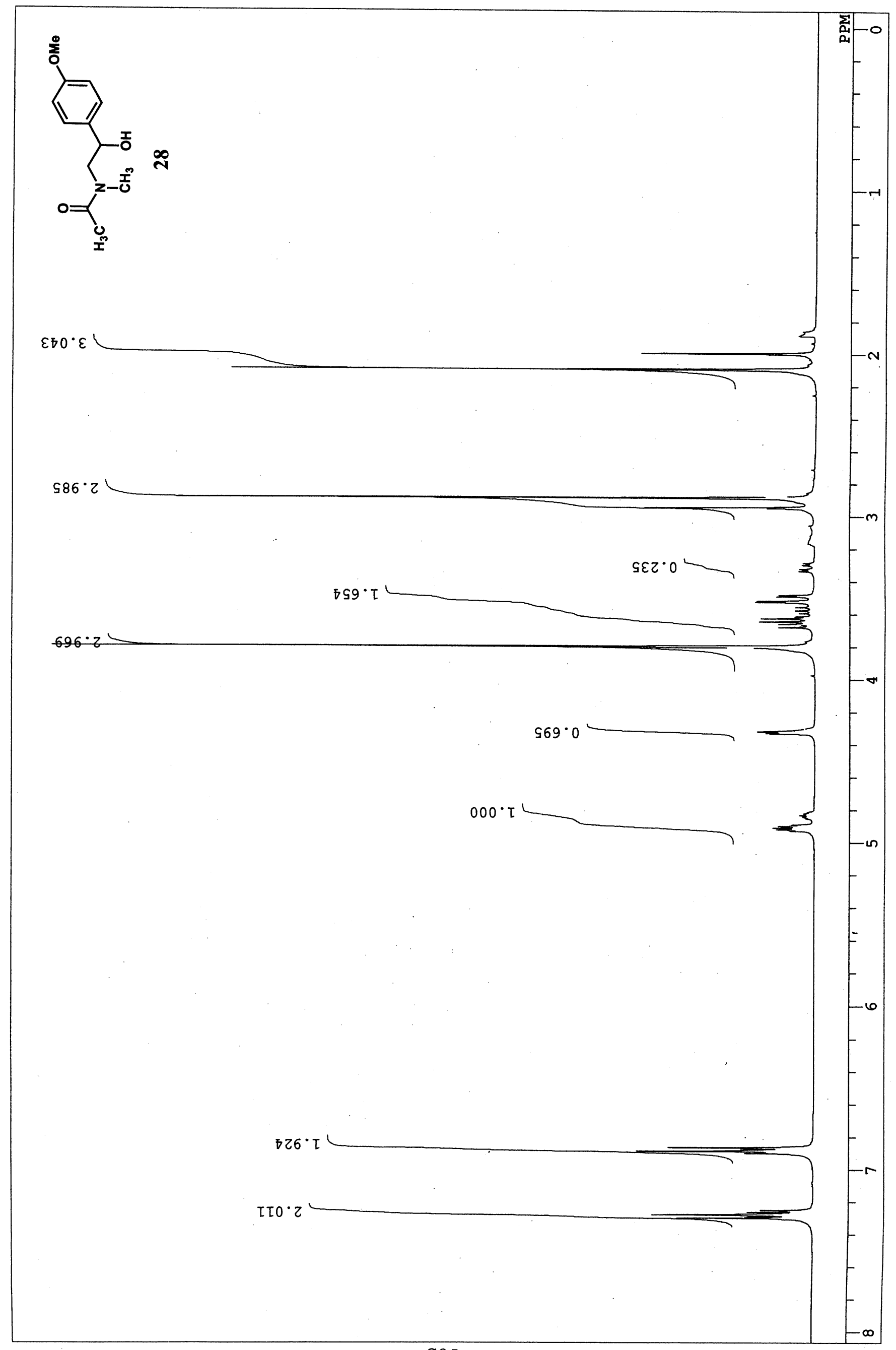




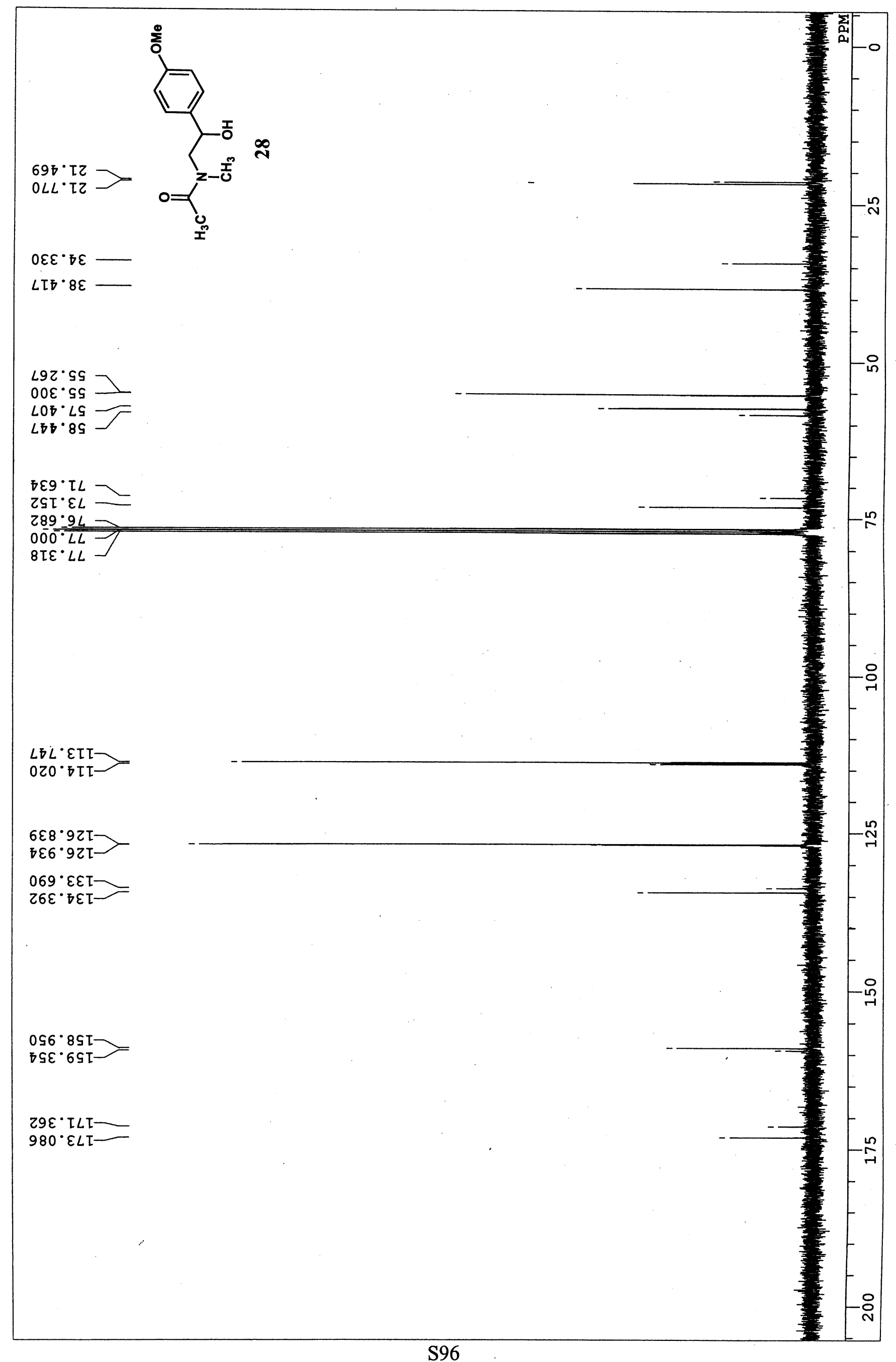

